\author{
ALL-FIBER PASSIVELY MODE-LOCKED FEMTOSECOND FIBER \\ LASERS \\ by \\ Jiaqi Zhou \\ B. Eng. (2009), M. Eng. (2012), Fudan University, CHINA \\ A dissertation \\ presented to Ryerson University \\ in partial fulfillment of the \\ requirements for the degree of \\ Doctor of Philosophy \\ in the Program of \\ Electrical and Computer Engineering
}

Toronto, Ontario, Canada, 2016

(C) Copyright by Jiaqi Zhou 2016 


\section{AUTHOR'S DECLARATION FOR ELECTRONIC SUBMISSION OF A DISSERTATION}

I hereby declare that I am the sole author of this dissertation. This is a true copy of the dissertation, including any required final revisions, as accepted by my examiners.

I authorize Ryerson University to lend this dissertation to other institutions or individuals for the purpose of scholarly research.

I further authorize Ryerson University to reproduce this dissertation by photocopying or by other means, in total or in part, at the request of other institutions or individuals for the purpose of scholarly research.

I understand that my dissertation may be made electronically available to the public. 


\title{
ALL-FIBER PASSIVELY MODE-LOCKED FEMTOSECOND FIBER LASERS
}

\author{
Jiaqi Zhou \\ Doctor of Philosophy \\ Electrical and Computer Engineering \\ Ryerson University
}

2016

\begin{abstract}
This dissertation presents three all-fiber designs of passively mode-locked lasers in order to achieve high pulse energy, environmentally-stable dissipative soliton (DS) operation in all-normal-dispersion cavities. A numerical model for DS mode-locked fiber lasers based on the nonlinear Schrodinger equation has been used to guide the experimental designs.
\end{abstract}

Firstly, an environmentally-stable and ultra-compact SESAM mode-locked fiber laser is demonstrated. The all-fiber design is realized using a mode-field-adaptor (MFA) to couple light onto the SESAM. A polarization-maintaining fiber loop mirror serves multiple functions as a highly reflective mirror, an output coupler and polarization selector. Self-starting and stable DS mode-locking operation is achieved with $1.7 \mathrm{~nJ}$ pulse energy and a $22 \mathrm{ps}$ pulse width. Secondly, an ultrastable DS mode-locking was demonstrated in a long cavity ring laser with a nonlinear amplified loop mirror (NALM) as a mode-locking device. The output 
pulses of 32 nJ, 615 fs de-chirped pulse width were obtained with the Raman signal suppressed below $-20 \mathrm{~dB}$ in a $81 \mathrm{~m}$ long cavity. The mode-locking is selfstarting and the mode-locked pulse train shows excellent stability. Thirdly, the mode-laser cavity was extended with a piece of large-mode-area (LMA) fiber with a low dispersion to further scaling up the pulse energy to $56.8 \mathrm{~nJ}$. The laser pulses were compressed to $750 \mathrm{fs}$ by a pair of volume gratings.

In the processing of scaling-up the pulse energy of the NALM mode-locked fiber laser, some interesting physical phenomena were observed, such as the operation regime transition from noise-like to DS with a sudden reduction of Raman signal and a unique waves-splitting with a stable temporal spacing. The phenomena were studied and explained in this dissertation.

In addition to the mode-locked fiber laser, a $\mathrm{CW}$ and a Q-switched fiber lasers were also designed with a single-mode- multimode- single-mode (SMS) filter as an effective mean of overcoming nonlinear effects. The transmission spectral property of the SMS was studied which fits well with theoretical calculation. One high efficiency SMS CW fiber lasers and one SMS Q-switched fiber laser were designed which showed the effectiveness of the SMS filter for inhibiting the SRS and significantly reducing SPM. 


\section{Acknowledgments}

It is a great pleasure for me to acknowledge many people who made this dissertation possible. First and foremost I would like to express my sincere gratitude to my supervisor, Dr. Xijia Gu for his expert supervision and guidance, continuous support and patience during the course of this work. I am grateful for the opportunity given by $\mathrm{Dr}$. Gu to be part of the Fiber Optics and Sensing Laboratory and study in an upcoming research area. For the past three years, his immense knowledge, patience, motivation and enthusiasm have contributed a lot to my personal growth. I could not have imagined having a better advisor for my Ph. D. study.

I also want to express my gratitude to Jiang $\mathrm{Li}$ and $\mathrm{Yi} \mathrm{Lu}$ for the help in assembling laser modules. Jiang's expertise in fiber optics, Bragg gratings, and fusion splicer is invaluable in successful completion of this work. My sincere thanks also go to Prof. Lian Zhao and Prof. Kaamran Raahemifar from whose lectures I benefited greatly. I also want to thank all the colleagues and friends from Fiber Optics and Sensing Laboratory for all the interesting discussions and fruitful comments and for making our laboratory a pleasant work place.

Last but not least I want to express my deepest love to my parents and my wife for their constant encouragement and endless support. Without their support, I would never reach this far. 


\section{Table of Contents}

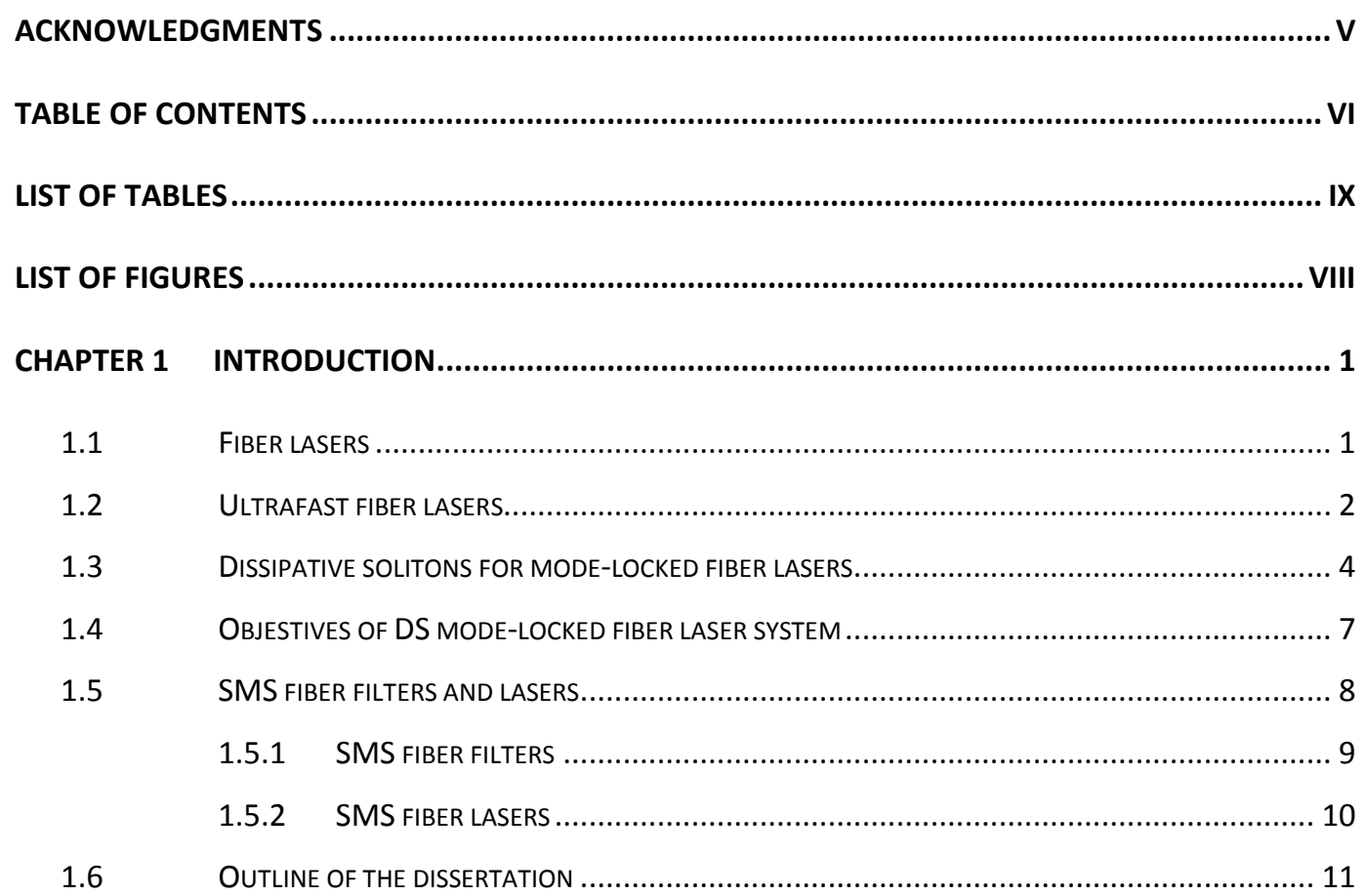

CHAPTER 2 DISSIPATIVE SOLITON MODE-LOCKING MECHANISMS ................................. 13

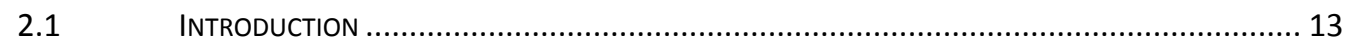

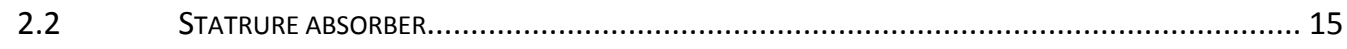

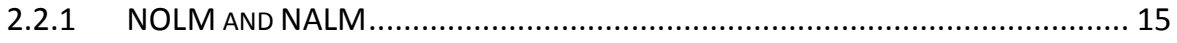

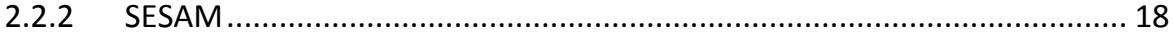

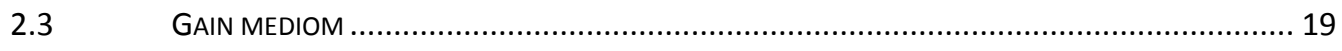

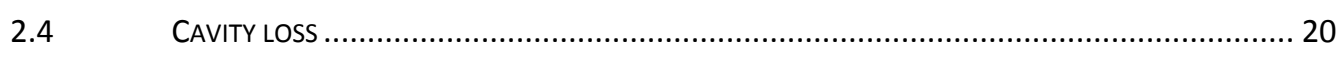

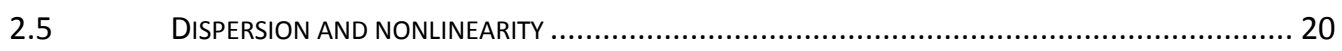

2.5.1 CHROMATICS DISPERSION................................................................. 21

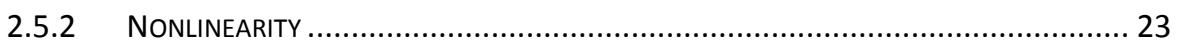

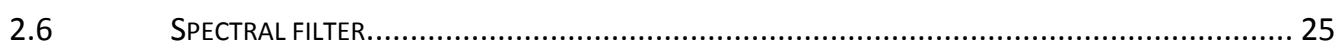

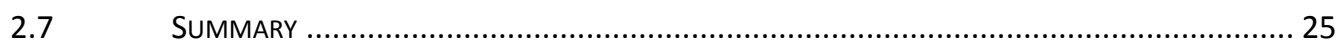

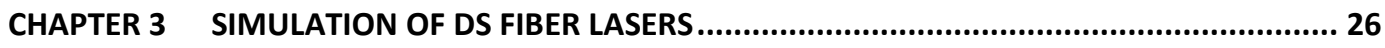

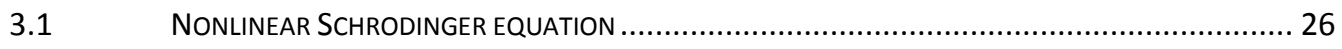

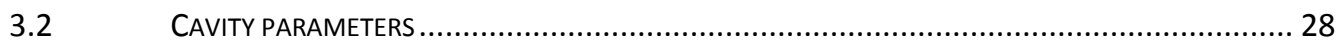




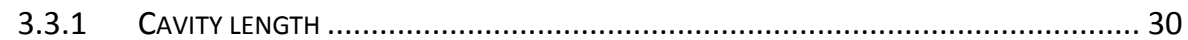

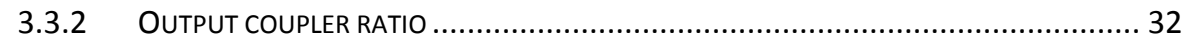

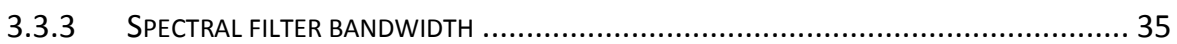

3.3.4 MAIN LOOP PUMP POWER ................................................................. 36

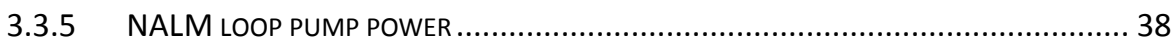

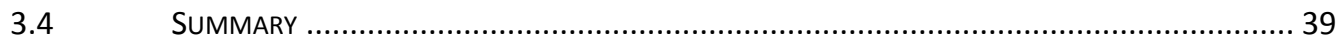

CHAPTER 4 SESAM MODE-LOCKED FIBER LASER........................................................... 41

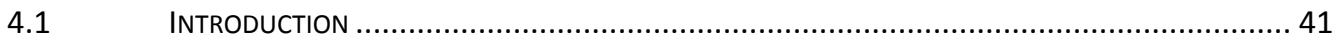

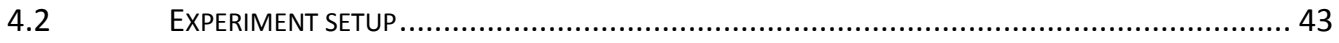

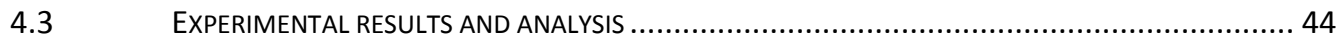

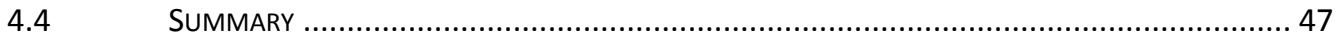

CHAPTER $5 \quad$ NALM MODE-LOCKED FIBER LASER ............................................................ 48

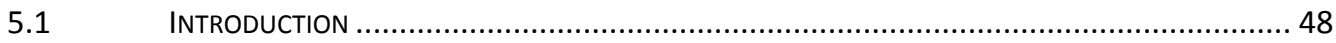

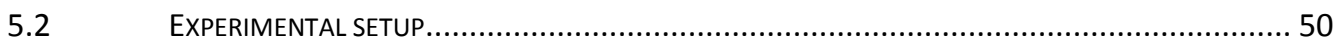

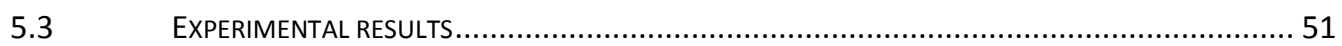

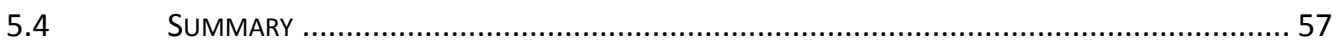

CHAPTER 6 GENERATION OF MULTIPLE DISSIPATIVE SOLITONS ...................................... 58

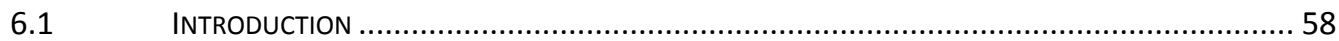

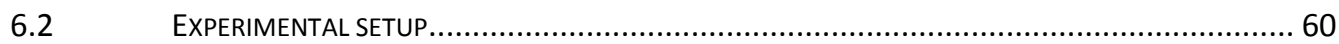

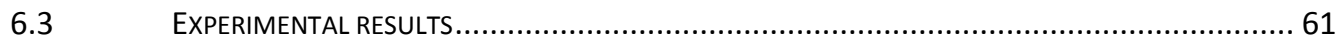

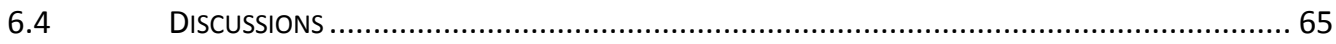

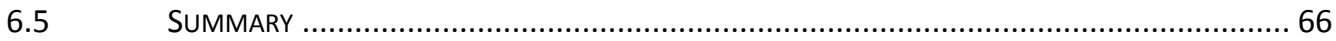

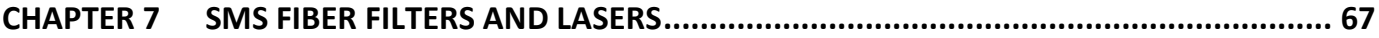

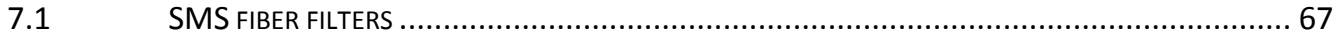

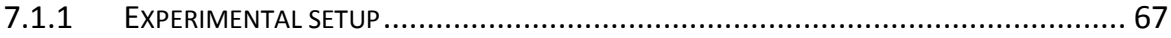

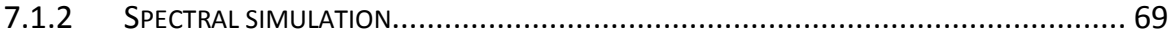

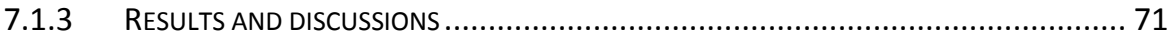

7.2 HIGH EFFICIENCY SMS FIBER LASER WITH DIFFRACTION LIMITED BEAM OUTPUT ..................... 73

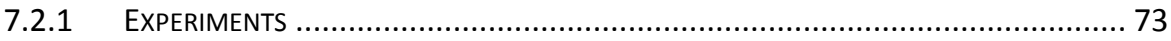

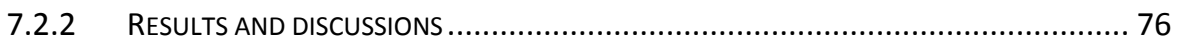

S.3 SMS Q-SWITCHED FIBER LASERS FOR INHIBITING NONLINEAR EFFECT ............................... 78 


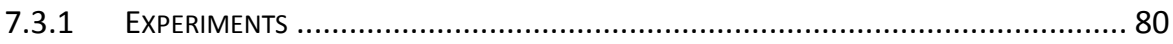

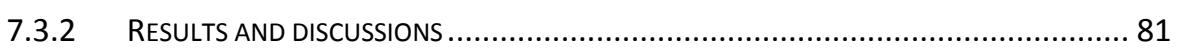

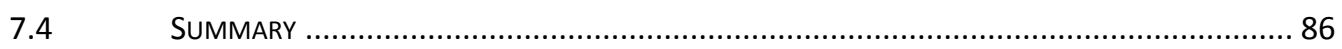

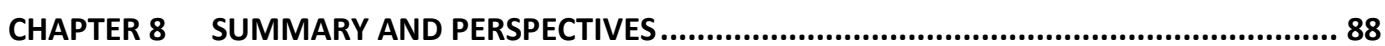

8.1 THE MAIN FEATURE OF MY DS MODE-LOCKED LASERS ............................................. 88

8.2 THE PERFORMANCE OF MY DS MODE-LOCKED LASERS................................................. 88

8.3 THE NEW PHENOMENA OBSERVED IN THE NALM MODE-LOCKED LASERS ............................. 89

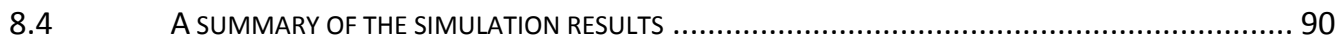

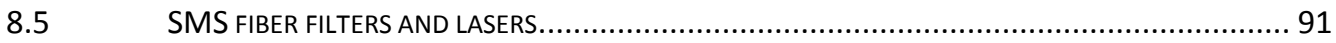

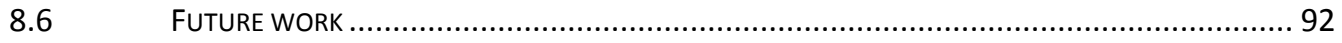

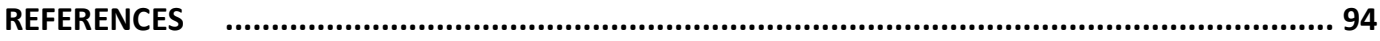




\section{List of Tables}

TABLE 1.1 SUMMARY OF RECENT DESIGNS FOR DS MODE-LOCKED FIBER LASERS..................................... 6

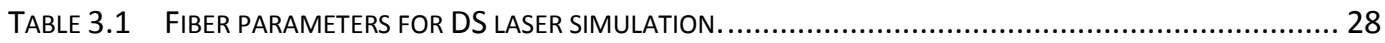

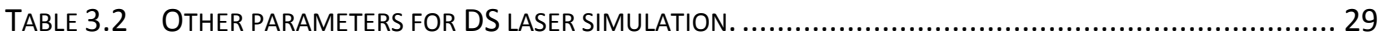

TABle 7.1 PARAMETERS COMPARISON BETWEen SMS LASER AND A REFERENCE LASER..............................85 


\section{List of Figures}

FIG. 1.1 LASER MACHINING WITH LONG-PULSE LASERS (A), SHORT-PULSE LASERS (B)........................... 3

FIG. 2.1 DIFFERENCES BETWEEN CONVENTIONAL SOLITONS AND DISSIPATIVE SOLITONS.......................... 14

FIG. 2.2 NONLINEAR OPTICAL LOOP MIRROR CONFIGURATION................................................... 15

FIG. 2.3 TRANSMISSION OF NOLM AGAINST INPUT POWER IN KILOWATT. ....................................... 17

FIG. 2.4 TRANSMISSION OF NALM AGAINST INPUT POWER IN KILOWATT ..................................... 18

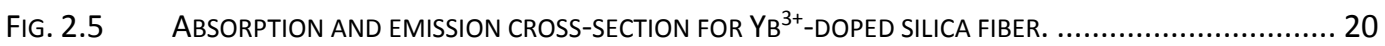

FIG. 2.6 VARIATION OF GROUP INDEX NG WITH WAVELENGTH FOR FUSED SILICA.................................... 22

FIG. 2.7 PLOTS OF A GAUSSIAN PULSE INTENSITY ENVELOPE (LEFT) AND CORRESPONDING CHIRP RAISED BY SPM

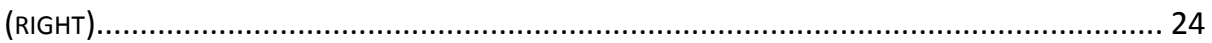

FIG. 3.1 SCHEMATIC OF YB ALL-FIBER NALM-BASED ANDI FIBER LASER AND TYPICAL NUMERICAL SIMULATION RESUlt: (A) AFTER THE LONG EXTRA PM FIBERS, (B) AFTER THE NALM, (C) AFTER THE FILTER. ........ 28

FIG. 3.2 PULSE DURATION EVOLUTION OF THE NALM-BASED ANDI FIBER LASER.................................... 30

FIG. 3.3 OUTPUT SPECTRUM AND PULSE SHAPE WITH 30 M EXTRA PM 980 FIBER (A, B); WITH 150 M EXTRA PM 980 FIBER (C, D).

31

FIG. 3.4 LASER PERFORMANCE VERSUS LENGTH OF EXTRA PM 980 FIBER: (A) PULSE WIDTH, (B) PULSE ENERGY, (C) PEAK POWER . 32

Fig. 3.5 OUTPUT SPECTRUM AND PULSE SHAPE WITH 50\% POWER OUTPUT (A, B); WITH 80\% POWER OUTPUT (C, D) 33

FIG. 3.6 LASER PERFORMANCE VERSUS OUTPUT RATIO: (A) PULSE WIDTH, (B) PULSE ENERGY, (C) PEAK POWER 34

FIG. 3.7 OUTPUT SPECTRUM AND PULSE SHAPE WITH DIFFERENT FILTER BANDWIDTH, WITH 2.2 NM (A, B) AND $4.2 \mathrm{NM}(\mathrm{C}, \mathrm{D})$ 35

FIG. 3.8 LASER PERFORMANCE VERSUS FILTER BANDWIDTH: (A) PULSE WIDTH, (B) PULSE ENERGY, (C) PEAK POWER .

FIG. 3.9 OUTPUT SPECTRUM AND PULSE SHAPE WITH DIFFERENT SATURATION ENERGY, WITH 0.7 NJ (A, B) AND $0.3 \mathrm{NJ}(\mathrm{C}, \mathrm{D})$; COMPARISON BETWEEN EXPERIMENT AND SIMULATION (A).

Fig. 3.10 LASER PERFORMANCE VERSUS SATURATION ENERGY: (A) PULSE WIDTH, (B) PULSE ENERGY, (C) PEAK POWER .

FIG. 3.11 OUTPUT SPECTRUM AND PULSE SHAPE WITH DIFFERENT NALM GAIN, WITH GNALM = 3 (A) AND GNALM $=5$ (B) 39

FIG. 4.1 SCHEMATIC DIAGRAM OF THE SESAM MODE-LOCKED FIBER LASER. 43 
FIG. 4.2 REFLECTIVITY SPECTRUM OF THE PM LOOP MIRROR............................................... 43

FIG. 4.3 OSCILLOSCOPE TRACES OF THE Q-SWITCHING PULSES AT A) 60 KHZ, AND B) $10 \mathrm{kHz} . \ldots \ldots \ldots \ldots \ldots . . . . . . . . .45$

Fig. 4.4 AUTO-CORRELATOR TRACE WITH AN OUTPUT POWER OF (A) $2.3 \mathrm{MW}$ AND (B) $21.7 \mathrm{MW} \ldots \ldots \ldots . . . .46$

FIG. 4.5 RADIO-FREQUENCY (RF) SPECTRUM (A) AROUND THE FUNDAMENTAL AND (B) HARMONIC REPETITION

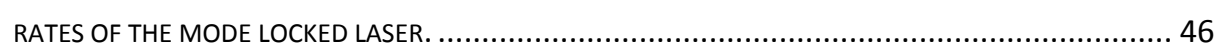

FIG. 4.6 OUTPUT POWER STABILITY TESTED WITH A DURATION OF 4 H. .............................................. 47

FIG. 5.1 SCHEME OF AN ALL-FIBER ALL-PM NALM DS FIBER LASER .......................................... 50

FIG. 5.2 SPECTRUM EVOLUTION OF THE NALM MODE-LOCKED LASER........................................ 52

FIG. 5.3 OUTPUT PULSE TRAIN OF THE NALM MODE-LOCKED LASER IN DS REGIME. ............................. 53

FIG. 5.4 RF SPECTRUM AT THE FUNDAMENTAL REPETITION RATE IN THE NOISE-LIKE REGIME (A), AND IN THE

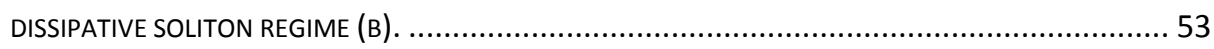

FIG. 5.5 INTEGRATED POWER OF THE NOISE-LIKE PULSES ( $\boldsymbol{\square}$ ), DS ( $\mathbf{\Delta}$ ) AND RAMAN EMISSIONS (O) VERSUS

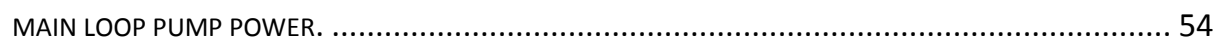

FIG. 5.6 SIMULATION OF NALM'S TRANSMITTED POWER AGAINST INPUT POWER. ............................... 55

Fig. 5.7 AutocorRelation tRACE OF THE MOdE-LOCKED LASER (A) NOISE-LIKE; (B) DS; AND (C) FULLY

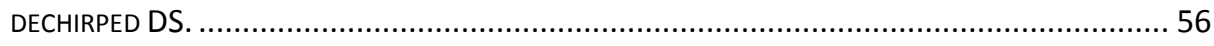

FIG. 5.8 OUTPUT POWER STABILITY TEST IN A DURATION OF 24 HOURS. .......................................... 56

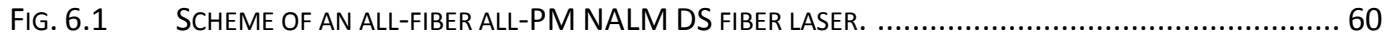

FIG. 6.2 DISPERSION MAP OF THE PM 085 LMA FIBER BY NUFERN. .............................................6 60

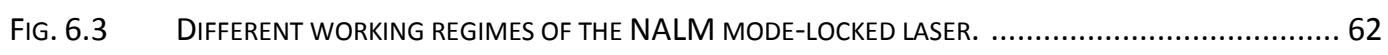

FIG. 6.4 SPECTRUM EVOLUTION OF THE NALM MODE-LOCKED LASER. .............................................6. 62

FIG. 6.5 OUTPUT PULSE TRAIN OF THE NALM MODE-LOCKED LASER IN DS REGIME WITH DIFFERENT NALM PUMP POWER (A) 377 MW, (B, E) 471 MW, (C, F) 477 MW, (D, G) 483 MW...................... 63

FIG. 6.6 RF SPECTRUM AT THE FUNDAMENTAL REPETITION RATE IN THE SINGLE PULSE DS REGIME (A), HARMONIC RF SPECTRUM OF THE SINGLE PULSE DS REGIME (B), HARMONIC RF SPECTRUM OF THE MULTI-PULSE DS REGIME WITH SEPARATING DISTANCE OF 20.2 NS (C), 40.6 NS (D), 83.9 NS (E),

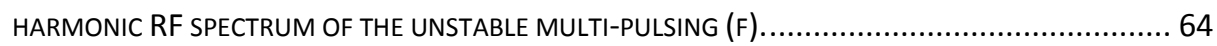

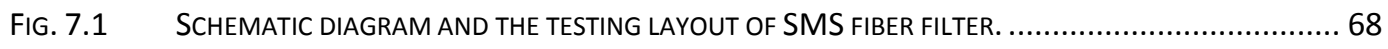

FIG. 7.2 TRANSMISSION SPECTRUM FROM 970 NM TO 1120NM WITHOUT STRAIN (SOLID LINE); AND

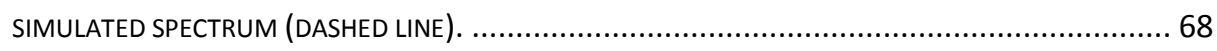

Fig. 7.3 CALCULATED PROPAGATION CONSTANT DIFFERENCE, $\triangle B$, BETWEEN THE LP01 AND LP02 MODES AS A FUNCTION OF WAVELENGTH. 71

FIG. 7.4 (A) WAVELENGTH SHIFT AS A FUNCTION OF STRAIN; AND (B) WAVELENGTH SHIFT AS A FUNCTION OF TEMPERATURE. 72 
FIG. 7.5 TRANSMISSION SPECTRA AT TWO DIFFERENT STRAIN LEVEL; AND (B) THE TRANSMISSION AT THE CRITICAL

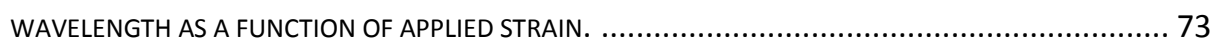

FIG. 7.6 TRANSMISSION SPECTRUM OF THE SMS FILTER WITH (A) A PASSIVE MULTIMODE FIBER WITH A CIRCULAR CLADDING; AND (B) A GAIN FIBER WITH AN OCTAGONAL CLADDING. THE INSERT SHOWS THE

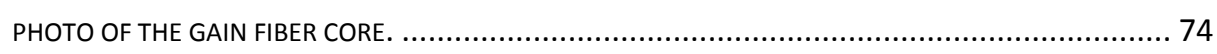

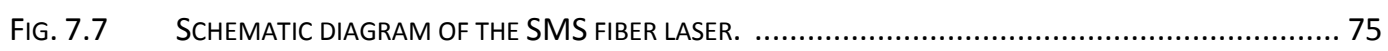

FIG. 7.8 (A) LASER OUTPUT POWER AS A FUNCTION OF PUMP POWER; AND (B) LASER EMISSION SPECTRUM WITH

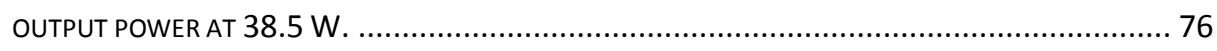

FIG. 7.9 OUTPUT LASER BEAM QUALITY OF SMS LASER MEASURED BY BEAM PROFILE AT THE OUTPUT POWER OF

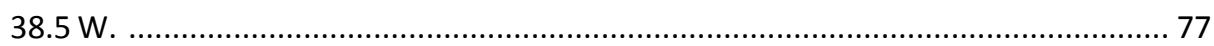

Fig. 7.10 OUtPUt LASER BEAM PROFILE FROM the MULTIMOde Fiber AT THE OUTPUT POWER OF 34.1 W. ... 78

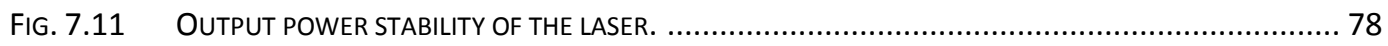

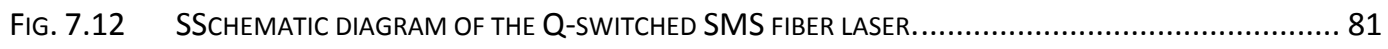

FIG. 7.13 (A) AVERAGE OUTPUT POWER VERSUS AVERAGE PUMP POWER; (B) EMISSION SPECTRUM OF THE LASER AT 100 KHZ IN 200 NM SPAN WITH THE INSET SHOWING THE SPECTRAL DETAIL. ...................... 82

Fig. 7.14 (A) OSCILLOSCOPE TRACE OF THE Q-SWITCHING PULSES AT $100 \mathrm{KHZ}$ RATE; (B) A SINGLE PULSE WITH

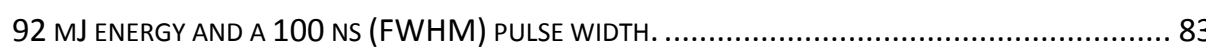

FIG. 7.15 (A) LASER SPECTRA AT DIFFERENT PEAK POWER; (B) SPECTRAL LINE WIDTH AS A FUNCTION OF PEAK

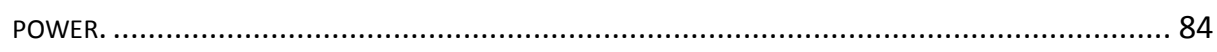

FIG. 7.16 A COMPARISON OF THE LASER EMISSION SPECTRA BETWEEN THE SMS STRUCTURED LASER AND THE REFERENCE LASER.

FIG. 7.17 THE SMS-STRUCTURED LASER PULSE WIDTH (BLACK); AND PEAK POWER (RED) AS A FUNCTION OF AVERAGE PUMP POWER. 


\section{Chapter 1}

\section{Introduction}

Ultrafast optics have for decades been a very remarkable research field, and for last ten years ultrafast mode-locked fiber lasers have found themselves numerous applications in fundamental researches as well as in industrial and medical applications, such as micromachining [1], optical imaging [2], fiber frequency combs [3], supercontinuum generation [4] and terahertz generation [5]. Among various types of mode-locked fiber lasers, dissipative soliton (DS) laser is one of the most attractive type due to its high pulse energy and short pulse width. Indeed, DS lasers have demonstrated the highest femtosecond pulse energy produced to date by the lasers made of standard fiber (up to $31 \mathrm{~nJ}$ output from a single oscillator has been achieved from a single-mode fiber) [6].

\subsection{Fiber lasers}

Optical fibers are well-known for confining light and sending it around bended loops. It was first invented for the application of medical endoscope in the 1950s, but the loss of fibers was relatively high at that time [7]. The major development of optical fiber started in 1960s and 1970s with its application mainly for longdistance data transmission. This technology continues to evolve rapidly to this day and for which Charles Kao was awarded the Nobel Prize in physics in 2009 [8]. However, the application of optical fibers to lasers, although demonstrated early on in 1961 [9], did not attract very much attention until the demonstration of erbium-doped fiber amplifiers and the development of high-brightness laser-diode pump sources in the 1980s $[10,11]$.

Before 1990's, classical solid-state lasers (lasers based on crystals like Ti: Sapphire and Nd: YAG) have dominated the market for decades, and in terms of 
reliability and long term stability, they are still the favorable choice. However, the working environment for mode-locked solid-stated lasers is somewhat strict, i.e. stable laboratory-like environments with optical tables and stabilized room temperature are often required. In addition, solid-state lasers have high power consumption, and often need regular maintenance [12]. On the contrary, fiber lasers are efficient, powerful and versatile waveguide resonant devices that have both advantages of glass optical fiber waveguide for optical gain and Fabry-Perot resonators for optical feedback. Cladding pumping using multimode semiconductor diode lasers is efficient and straightforward. It excites the gain medium in the single-mode core of the fiber to generate near-diffraction-limited output with the brightness enhancement of at least two orders of magnitude than the pump diode. Furthermore, passively air-cooling of optical fiber is simple in design owing to its large surface-area-to-volume ratio $[12,13]$. Among all types of fiber lasers, ultrafast fiber lasers have gained most interest because of their widely-expanded applications in fiber communications, fundamental science and industry.

\subsection{Ultrafast fiber lasers}

Interest in ultrafast fiber lasers initially developed from the demand for increasingly shorter pulses in fiber communications as well as from the stability of ultrafast fiber lasers because of their highly integrated designs in the early 1990s [14, 15]. Since those early days, ultrafast fiber lasers have been making continuous progress and expansion in their applications. Currently, there are more than 40 companies selling short-pulse fiber lasers for a range of applications, from micromachining, medical imaging, to precision metrology. In particular, micromachining based on ultrafast fiber lasers is highly demanded in the fabrication processes like drilling, cutting and marking. Fiber lasers with pluses shorter than 10 picoseconds is required for the machining of glass, ceramics and semiconductors, for the purpose of avoiding thermal microcracks [16]. Fig. 1.1(a) shows schematically the thermal microcracks caused by long pulse laser, whose 
pulse width is several ns. When the beam incidents on the surface of a substrate, the material melts down and the heat begins to dissipate into the material. Pieces of melted debris are thrown out into the beam, causing scattering of the laser. The rapid thermal transition causes microcracks to form in the bulk of the material. Fig. 1.1(b) shows the short pulse machining by which the thermal microcracks can be avoided [17].

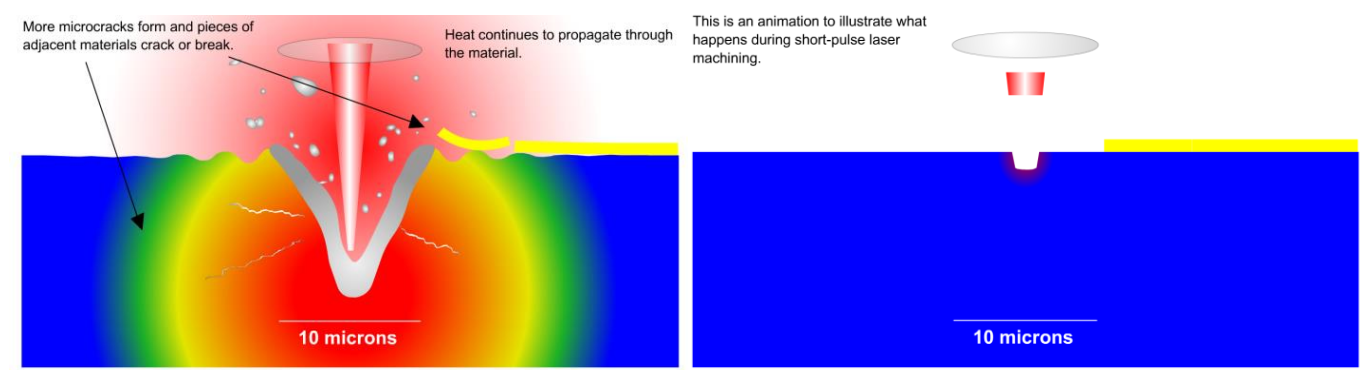

Fig. 1.1 Laser machining with long-pulse lasers (a), short-pulse lasers (b) [17]

The key properties that makes ultrafast fiber lasers attractive are its gain media, the rare earth doped fibers, which have high single pass gain, broad gain bandwidths and excellent beam quality. Ytterbium-doped silica fibers are of particular significance since they combine properties like high optical conversion efficiency, broad gain bandwidth, and large saturation fluence, offering an almost ideal gain medium for the generation and amplification of potentially high power pulses. According to the Fourier transform, the narrower the pulse width is, the broader the spectrum would be. Therefore, in order to generate femtosecond pulses, Full width at half maximum (FWHM) of the spectrum should be in the tens of $\mathrm{nm}$. Such requirement can be well-satisfied by the broad gain bandwidth of Ytterbium. In the past ten years, a large contribution to the development of ultrafast fiber lasers comes from the enhancement of the pulse energy of DS fiber lasers. DS is generated in the normal dispersion region of silica fiber, which matches the emission wavelength region of Ytterbium. This gives Ytterbium superiority to Erbium for generating DS pulses. 


\subsection{Dissipative solitons for mode-locked fiber lasers}

Introduced in 1965 by Zabusky and Kruskal, the term 'soliton' was originally used to refer to localized solutions of integral nonlinear systems. Such solutions are remarkable because they maintain their shape and velocity during the propagation, and remain intact when interacting with radiation waves [18]. In optics, conventional solitons result from the single balance between self-phase modulation (SPM) and dispersion. However, applications of soliton fiber lasers have been limited because of their low pulse energies [6]. In order to balance the SPM, such kind of fiber lasers are constructed entirely of fiber with anomalous group velocity dispersion (GVD), generating solitons as short as $\sim 200 \mathrm{fs}$ in duration, with the pulse energy restricted by the spectral sidebands and soliton area theorem to $\sim 0.1 \mathrm{~nJ}$ [19]. Pulse energy of up to several $\mathrm{nJ}$ can be obtained when the laser has segments of normal and anomalous GVD [20, 21]. In that case, the pulse breathes (i.e., the pulse width varies periodically according to different segments of normal and anomalous GVD fibers) as it traverses in the cavity.

The segments of normal and anomalous GVD fiber are both employed in many femtosecond fiber lasers before 2006, until Frank Wise et al. at Cornell University designed the first dissipative soliton (DS) fiber laser by employing all-normaldispersion (ANDi) structure [23]. The concept of DS can be traced back to the early 1990s, when physicists found out that solitary waves did also exist in a wide range of non-integrable and non-conservative systems [24, 25]. New terminology was needed to describe solitary waves with nonlinear gain or loss mechanisms, thereafter referred to as DS, which is the localized formation of an electromagnetic field that are balanced through an energy exchange with the environment in the presence of nonlinearity and dispersion [6]. By designing the laser cavity using fiber with large normal GVD, Wise et al. used one frequency filter to stabilize mode-locked operation characterized by cutting off the everbroadening highly-chirped pulse width. According to their papers, stable and selfstarting pulses were generated with energy as high as $20 \mathrm{~nJ}$, and pulses can be de- 
chirped to 200 fs pulse width [20]. Femtosecond pulses with peak powers near $100 \mathrm{~kW}$ are thus available from this DS mode-locked fiber laser configuration.

In order to achieve self-starting DS mode-locked fiber laser, different stature absorbers (SA) or equivalent SAs have been used. These include semiconductor saturable absorption mirror (SESAM) [26], carbon nanotube (CNT) [27], atomic multilayer graphene [28], nonlinear polarization rotation (NPR) [29], nonlinear optical loop mirror (NOLM) [30] and nonlinear amplified loop mirror (NALM) [31]. However, each of these techniques has its advantages and drawbacks. The $\mathrm{CNT}$ and grapheme methods have recently been intensively investigated. They offer the advantages of easily controllable saturable absorption strength, excellent environmental stability, and ultrafast saturable absorption but suffers from low damage threshold that leads to the limited pulse energy. The saturable absorption wavelength of a CNT is determined by its tube diameter. To mode-lock lasers with different operation wavelengths, CNTs with different tube diameters are needed. The SESAM method has the advantages of being easy to employ and intrinsically environmental stable, but the SESAM itself is challenging to fabricate and its damage threshold is relatively low and long-term reliability uncertain. Besides, for lasers operating at different wavelengths, different SESAMs need to be designed and used. The NPR technique is based on the nonlinear polarization delay at two axes in the laser cavity. It is independent on the laser operation wavelength, easy to implement, and has ultrafast saturable absorption. However, the methods are intrinsically environmentally unstable since polarization controllers are often used.

Although many designs of the DS mode-locked fiber lasers have been reported, only a few of them were in all-fiber structures [32-34]. Many DS lasers with high performance such as reported in [20,27-31] contain some bulk components. For example, Wise et al. have reported a NPE-based DS laser in which the NPE is implemented with two quarter-waveplates, a half-waveplate, and a polarizing beam splitter. Stable and self-starting pulses are generated with energies above 20 
nJ [20]. However, when they demonstrated an all-fiber DS laser by replacing the NPE with single-walled carbon nanotubes (SWCNTs) as an SA, the pulse energy was reduced to $3 \mathrm{~nJ}$, which was substantially lower compared to that of DS laser with bulk optics [33]. Although non-all-fiber structure enjoys a higher performance, they have drawbacks such as large foot-print and requirement of frequent alignment. Therefore, all-fiber versions of the normal-dispersion DS lasers have much better potential for applications. The performance of those DS lasers are compared in Table 1.

Table 1.1: Summary of recent designs for DS mode locked fiber lasers.

\begin{tabular}{|c|c|c|c|c|c|c|c|}
\hline Ref. \# & 26 & 32 & 37 & 38 & 27 & 34 & 29 \\
\hline Pulse width (ps) & 4.5 & - & 30 & 70 & 24 & 11.7 & 49 \\
\hline $\begin{array}{l}\text { After dechirp } \\
\text { (fs) }\end{array}$ & 80 & 230 & 200 & 300 & - & - & - \\
\hline $\begin{array}{l}\text { Repetition rate } \\
\quad(\mathrm{MHz})\end{array}$ & 70 & 34 & 7 & 1.7 & 25.8 & 22.7 & 13.3 \\
\hline $\begin{array}{l}\text { Average power } \\
\quad(\mathrm{mW})\end{array}$ & 2200 & 51 & 140 & 42.5 & 53 & 50 & 30.6 \\
\hline $\begin{array}{l}\text { Pulse energy } \\
\text { (nJ) }\end{array}$ & 31 & 1.5 & 20 & 25 & 2.1 & 2.2 & 2.3 \\
\hline $\begin{array}{c}\text { Center } \\
\text { wavelength } \\
(\mathrm{nm})\end{array}$ & 1060 & 1030 & 1015 & 1015 & 1035 & 1990 & 1585 \\
\hline Laser cavity & ring & ring & ring & ring & linear & linear & ring \\
\hline $\begin{array}{l}\text { Mode-locking } \\
\text { method }\end{array}$ & NPR & NPR & NPR & NPR & SESAM & SESAM & graphene \\
\hline
\end{tabular}

Recently, Broderick et al. reported an all-fiber DS mode-locked laser with an NALM which achieved environmental-stable output with pulse energy up to $16 \mathrm{~nJ}$ [35], much higher than those of SEASAM mode-locked lasers. The linearly chirped pulse can be compressed to a few hundred fs level after external compression. In their ring-cavity, a long single-mode fiber, whose dispersion stretched the pulse width linearly before being amplified, was inserted between 
the gain fiber and NALM. This all-fiber all-PM structure appears to be an ideal solution to achieve an environmentally-stable, compact, and high energy ultrafast laser [36].

Table 1.1: Summary of recent designs for DS mode locked fiber lasers (continued).

\begin{tabular}{|c|cccccc|}
\hline Ref. \# & 28 & 33 & 30 & 31 & 35 & 36 \\
\hline Pulse width (ps) & 5.8 & 1.5 & 3.63 & 7.6 & 68 & 19 \\
After dechirp (fs) & 226 & 235 & - & 344 & 370 & 120 \\
$\begin{array}{c}\text { Repetition rate } \\
(\mathrm{MHz})\end{array}$ & 23.6 & 50 & 3.278 & 10 & 1.7 & 10 \\
$\begin{array}{c}\text { Average power } \\
\quad(\mathrm{mW})\end{array}$ & 55.6 & 155 & 5 & 3 & 27 & 42 \\
$\begin{array}{c}\text { Pulse energy (nJ) } \\
\text { Center wavelength } \\
(\text { nm) }\end{array}$ & 1560 & 1030 & 1572 & 1030 & 1030 & 1030 \\
$\begin{array}{c}\text { Laser cavity } \\
\text { Mode-locking } \\
\text { method }\end{array}$ & ring & ring & ring & ring & ring & ring \\
\hline
\end{tabular}

\subsection{Objectives of the DS mode-locked fiber laser systems}

Although DS mode-locked fiber lasers have many advantages over classical solidstate ultrafast lasers, there are also many challenges for the DS laser to overcome in order to overtake the solid state ultrafast lasers.

One of the challenges is to achieve self-start, environmentally-stable modelocking. The bulk components used in either NPE or for coupling of light to SESAM might cause output power instability, which also diminish the advantages of the fiber lasers (free from aliment). Meanwhile, environmentally stable means that the output from the fiber laser is not susceptible to changing environmental conditions like air flow, temperatures or the vibration of optical platforms. 
Another challenge is to further scale up the pulse energy. Recent developments of all-PM-fiber laser mode-locked by NALM [31, 35, 36] or NPE with a short piece of SMF $[37,38]$ resulted in the stable DS with energies of up to $20 \mathrm{~nJ}$ as shown in Table. 1.1. However, further energy scale-up is found to be limited by stimulated Raman scattering (SRS), which converts the excess energy of DSs to a noisy pulse at red-shifted Stokes wavelengths.

The main objectives of this research is to demonstrate an environmental-stable, compact-size DS fiber laser with a high pulse energy of over $50 \mathrm{~nJ}$ and a pulse width of several hundred fs for practical industrial applications. An all-fiber allPM cavity structure is applied to achieve environmental-stable and compact-size and large-mode-area fiber is used to extend the cavity length while reducing dispersion and nonlinearity. NALM is used as both an equivalent SA and an amplifier to realize self-starting high energy mode-locked output.

\subsection{SMS fiber lasers}

Another objective of this dissertation is to describe a single-mode-multimodesingle-mode (SMS) filter and its applications in fiber lasers. In order to overcome the energy limitation caused by nonlinear effects one solution is to use largemode-area (LMA) fiber as the gain medium in fiber lasers. SMS was originally designed for fiber filters which uses mode interference to achieve certain spectral filtering [39]. The light from the single-mode fiber couples into many modes in the multimode fiber and the interference of these modes results in a period intensity distribution along the fiber axis. If an output single-mode fiber is spliced at an interference maximum node, a transmission maximum will be obtained for certain wavelength. SMS fiber structures have been employed as optical sensors for strain, temperature and refractive index measurements and fiber lasers for obtaining high efficiency while still maintaining single-mode output. 


\subsubsection{SMS fiber filters}

Since the demonstration of SMS band pass filters in 2006 [40], many fiber sensors have been developed for strain, temperature and refractive index measurements using their spectral features [41]-[43]. The SMS-based sensors are attractive since their spectral response to strain and temperature is of similar magnitude as that of fiber Bragg grating (FBG), and their structure is very simple. In recent years, LMA fibers have also attracted significant attention due to their extensive applications in high power fiber laser. When a section of LMA fiber is inserted to replace the multimode fiber in an SMF filter, only a few modes could be excited so the transmission spectrum of the SMS filter exhibits periodic peaks. If only two modes are mainly excited, and the propagation constants of the two modes, $\beta_{1}$ and $\beta_{2}$, changes very slowly with the wavelength, the transmission spectrum will be sinusoidal, similar to that of classical two beam interference. In a very special case, the difference between $\beta_{1}$ and $\beta_{2}, \Delta \beta$, can reach a maximum at certain wavelength $\lambda_{0}$, which leads to an interesting interference spectral fringe pattern: the fringe spacing increases with the increasing wavelength till it reaches a critical wavelength, $\lambda_{0}$; after that the fringe spacing decreases with the increase of wavelength. Near $\lambda_{0}$, the interference shows either a broad peak or a trough depending on the phase of interference. The observation of this critical wavelength was reported by Tripathi et al. near $1300 \mathrm{~nm}$ in an SMS filter, in which, the multimode fiber has a staircase refractive index profile [44]. The phenomenon was also reported by Salik et al., in C-band, for an SMS filter whose multimode fiber was a piece of dispersion compensating fiber supporting a few modes [45]. Since the transmission at $\lambda_{0}$ is sensitive to either strain or temperature, some sensing applications were proposed and successfully demonstrated. A filter with a variable transmission is very useful, for example, it can be used as a variable output coupler for output power optimization of a fiber laser. However the SMS filters reported in [44], [45] operate in $1300 \mathrm{~nm}$ and in C-band respectively, and used specialty fibers. In chapter 7 of this dissertation, a design for an SMS filter with maximum propagation constant difference near 
$1046 \mathrm{~nm}$ and made of a commercially available step-indexed LMA fiber will be presented.

\subsubsection{SMS fiber lasers}

In the development of fiber lasers, power scaling is an important topic due to the increasing demand on high power laser systems in material processing and other applications [46]. Although more high power pump diodes have become available, their output typically couples into a multimode fiber; the higher the power, the larger the required core diameter. When this fiber-coupled laser diode is used to pump $\mathrm{Yb}$-doped gain fiber, a small core/cladding diameter ratio of the gain fiber leads to low absorption of pump power which results in a long gain fiber. At high power, a long gain fiber will induce nonlinear effects such as stimulated Brillouin scattering and Raman scattering that limit the power scaleup. Both problems can be solved if the ratio of the core/cladding diameters of the gain fiber can be increased. Recently some fiber manufacturers started to offer the gain fibers with core/cladding diameters of $15 / 130 \mu \mathrm{m}, 20 / 130 \mu \mathrm{m}$ and even $30 / 130 \mu \mathrm{m}$. Nevertheless, increasing core size will be detrimental to the beam quality of the fiber laser. As the V-number of the fiber increases beyond 2.405, the high order modes in the fiber laser could also be excited. Although singlemode propagation can still be maintained if the numerical aperture (NA) can be reduced. However, due to the fabrication limitation, it is difficult for a step-index fiber to reduce its core NA to below 0.06. Some newly developed photonics crystal gain fiber can achieve large core size and a low NA, however splicing this fiber with other fiber often introduces a large loss and the cost of this fiber is high [47]. There had been other methods developed to achieve high output power while maintaining diffraction-limited beam output. Some of them involved specialty active fiber design, such as a special refractive-index profile to achieve $\mathrm{M}_{2}<2.0$ beam quality [48], ultra-short cavity length with highly doped fiber [49], large-core phosphate fiber with an intra-cavity spatial-mode filter [50], helicalcore fibers with a core diameter of $30 \mu \mathrm{m}$ [51] and leakage channel gain fiber of 
$3160 \mu \mathrm{m}^{2}$ effective core area [52]. The disadvantages of these methods are the use of specially designed large-mode-area (LMA) fibers that are not commercially available. If a new design can be found that uses a step-indexed large core gain fiber while still maintaining Gaussian-shaped output beam profile, it would be interesting to many researchers in this field.

Peyghambarian et al. reported fiber lasers and amplifiers based on SMS structure $[53,54]$. An LMA fiber of $25 \mu \mathrm{m}$ core diameter was used as the gain fiber which was spliced to an SMF28 fiber for output. Although the laser achieved diffractionlimited output, its slope efficiency is only $8.1 \%$ and an output power of $1.1 \mathrm{~W}$ at $1535 \mathrm{~nm}$. Whether SMS structure can be used for high power fiber laser with high efficiency is a subject that needs to be studied. In chapter 7, a high efficiency SMS CW fiber lasers and an SMS Q-switched fiber laser to suppress nonlinear effect will be presented.

\subsection{Outline of the dissertation}

In Chapter 2, the nonlinear Schrodinger equation is described, and the physical background for pulse propagation in optical fibers is presented.

In Chapter 3, a numerical model for DS mode-locked fiber laser is developed. Stable DS mode-locking with various cavity parameters are simulated in order to optimize the laser cavity design.

Chapter 4 presents an all-fiber, environmentally-stable and ultra-compact ANDi fiber laser by using one mode-field-adaptor (MFA) to launch light onto the SESAM. Self-starting and stable dissipative solitons mode-locking operation is achieved with $1.7 \mathrm{~nJ}$ pulse energy and 22 ps pulse width.

Chapter 5 shows that stable DS mode-locking can be achieved in a long cavity ring laser with an NALM. $32 \mathrm{~nJ}, 650 \mathrm{fs}$ de-chirped pulse width were obtained in the presence of a relatively high Raman signals. 
In Chapter 6, a unique multi-pulsing phenomenon is investigated in the NALM dissipative soliton mode-locked fiber laser.

Chapter 7 presents SMS filter design and characterization. The applications of the SMS filter in two laser designs are also described.

Chapter 8 Summary and outlook. 


\section{Chapter 2}

\section{Dissipative Soliton Mode-locking Mechanisms}

The forming of a stable dissipative soliton is the result of a balance between gain, loss, nonlinearity and dispersion. Thus one typical all-normal dispersion modelocked fiber laser usually consists of several modules, including gain medium, pump source, output coupler, band pass filter and saturated absorber [6]. Each module is essential and plays certain role in the generation of DS. In this chapter, I will go through each factors, which affect the DS mechanism, in order.

\section{$2.1 \quad$ Introduction}

In optics, conventional solitons are the result of a balance between nonlinearity (i.e. SPM (self-phase modulation)) and dispersion. Such solitons are remarkable because they could maintain their shape and velocity when propagating in fibers. However, applications of soliton fiber lasers have been limited because of their low pulse energies [19]. Therefore, the purpose of energy scale-up leads to a different mechanism of solitons - dissipative solitons. In contrast with conventional solitons, in which a given set of parameters and initial conditions for the propagation equation generally leads to an infinite number of soliton solutions, the solution in a dissipative system is always a fixed one [6]. Moreover, the energy, pulse chirp and pulse shape of a DS are predetermined by the cavity parameters, rather than by the initial conditions, as shown in Fig 2.1. Thus, the mode-locked fiber lasers based on DS enjoy highly stability because of these features, which makes these laser attractive in industrial applications.

As shown in Fig 2.1, the forming of a stable dissipative soliton (DS) is the result of a balance between gain, loss, nonlinearity and dispersion. When an optical pulse is propagating though a piece of fiber, its temporal and spectral evolution 
can be described by the nonlinear Schrodinger equation (NLSE). The NLSE has been used extensively to model pulse propagation in optical fibers [58-60] since early 1980s. A typical normalized NLSE could be written as:

$$
\frac{\partial A(z, \tau)}{\partial z}=-i \frac{\beta_{2}}{2} \frac{\partial^{2} A(z, \tau)}{\partial \tau^{2}}+i \gamma|A(z, \tau)|^{2} A(z, \tau)-\frac{1}{2}(g-\alpha) A(z, \tau)+\frac{g T_{2}^{2}}{2} \frac{\partial^{2} A(z, \tau)}{\partial \tau^{2}}
$$

where the optical field envelope $\mathrm{A}$ is a function of $\mathrm{z}$ and $\mathrm{t}$, while $\mathrm{z}$ is the propagation distance and $t$ is measured in a frame of reference moving with the pulse at the group velocity $v_{g}\left(t=\tau-z / v_{g}\right)$. The first two terms on the right side represent group velocity dispersion (GVD) and SPM respectively. The third one is gain minus loss, while the last one is gain dispersion. For the traditional solitons, anomalous GVD can balance SPM in term of the chirp and produce a stable solution for NLSE. However, for ANDi fiber lasers, both normal GVD and SPM have a positive chirp. Thus, extra loss mechanism (spectral filter) is required to prevent the pulse from broadening. Therefore, one typical ANDi mode-locked fiber laser usually consists of several modules, such as gain medium, output coupler, band pass filter and SA. Each module is essential and plays a critical role in the generation of DS. The following sections will go through the basic function of each module. Simulations and more detailed explanations of NLSE will be included in chapter 3.

\section{Conventional}

solitons system

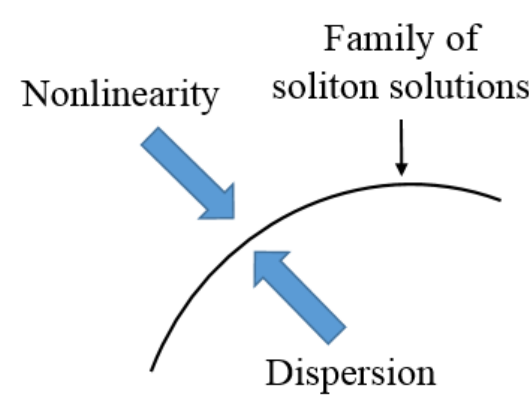

Dissipative

solitons system

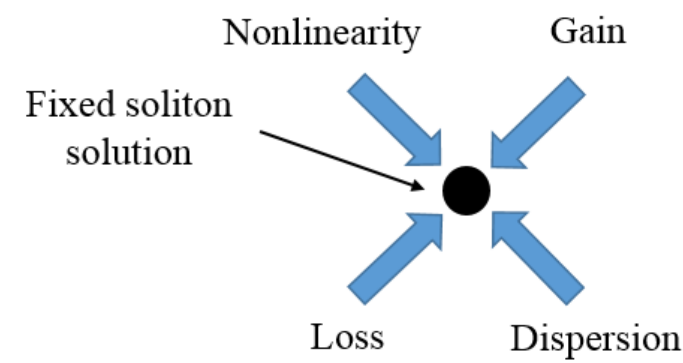

Fig. 2.1 Differences between conventional solitons and dissipative solitons. 


\subsection{Stature Absorber}

Self-starting is one of the most important properties of the industrialized ultrafast fiber lasers. In order to achieve self-starting, a saturable absorber is used to obtain a self-amplitude modulation of the light inside the laser cavity. These absorbers introduce some loss to the intra-cavity laser radiation, which is relatively large for low intensities but significantly smaller for a short pulse with high intensity. Thus, a short pulse could produce a modulation of loss because the high intensity at the peak of the pulse saturates the absorber more strongly than its low intensity wings, by which self-starting mechanism is achieved $[13,61]$. Various saturable absorber (or equivalent SA) mechanisms can be used to achieve stable modelocking. In this section, two of them will be introduced. One is nonlinear optical loop mirror (NOLM) and NALM; the other is semiconductor saturable absorber mirror (SESAM).

\subsubsection{NOLM and NALM}

NOLM can be used as a switching device, which operates on the nonlinear phase induced by SPM $[62,63]$. Its advantage is that it does not require interferometric alignment, is robust, and is simple to construct. In addition, the device can exploit soliton-propagation effects to give excellent switching characteristics for ultrashort pulses, which acts as a great stature absorber.

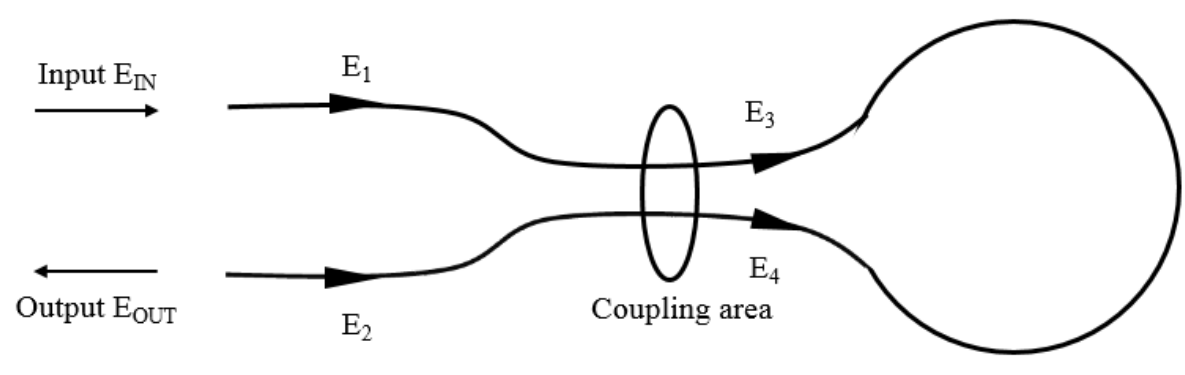

Fig. 2.2 Nonlinear optical loop mirror configuration. 
Fig. 2.2 shows the configuration. The device is based on one $2 \times 2$ coupler with power-coupling ratio $\alpha$ : $1-\alpha$. When two ends are spliced together, the coupler operates like a mirror. If the port 1 is taken as the input, $\mathrm{E}_{\mathrm{IN}}$, then the output at port 2 can be written as:

$$
\left|E_{02}\right|^{2}=\left|E_{I N}\right|^{2}\left(1-2 \alpha(1-\alpha)\left\{1+\cos \left[(1-2 \alpha)\left|E_{I N}\right|^{2} \times 2 \pi n_{2} L / \lambda\right]\right\}\right)
$$

This equation shows that for any value of $\alpha \neq 1 / 2,100 \%$ of the power emerges from port 2 whenever

$$
n_{2} 2 \pi|E|^{2} L / \lambda=m \frac{\pi}{1-2 \alpha}
$$

for $\mathrm{m}$ odd. The minimum power occurs for $\mathrm{m}$ even and can be expressed as below:

$$
\left|E_{02}\right|^{2}=\left|E_{I N}\right|^{2}(1-4 \alpha(1-\alpha))
$$

The best switching ratio (i.e., the contrast between the linear off and the higherintensity on) occurs for a closest to 0.5 , but the switching energy increases correspondingly. Fig. 2.3 shows the transfer function from port 2 as a function of input power for two values of $\alpha$. The transmission is calculated by using Eq. (2.2) with $\mathrm{n}_{2}=3.2 \times 10^{-16} \mathrm{~cm}^{2} / \mathrm{W}$, an effective area of $30 \mu \mathrm{m}^{2}$, loop length $\mathrm{L}=15 \mathrm{~m}$, and $\lambda=1.55 \mu \mathrm{m}$.

The design of a NOLM involves a competing issue between self-starting ability and modulation depth. For fixed loop length the largest modulation depth occurs for a coupling ratio closest to 50, but the highest input peak power is required to reach the first maximum transmission peak. On the other hand, smaller peak power is required to reach the first maximum in a NOLM biased further from equal splitting, but less modulation depth is obtained. Self-starting of mode locking is generally required for practical applications. To reduce the threshold requirement for self-starting while maintaining adequate modulation depth, one 
may choose an 80:20 coupler. Compared to the commonly used 50:50 coupler, an 80:20 coupler produces a much-steeper increase in transmission at low intensity, while it maintains adequate modulation depth. The former is required for selfstarting operation, and the latter is required for effective suppression of the background.

The main disadvantage of NOLM-based fiber lasers is that the modulation depth of the NOLM cannot be adjusted unless one turns off the laser and changes the NOLM fiber loop length. Besides, the total cavity length of the NOLM-based fiber laser is often quite long due to the long fiber of the NOLM loop for the purpose of self-starting. These problems can be avoided in the NALM-based fiber lasers.

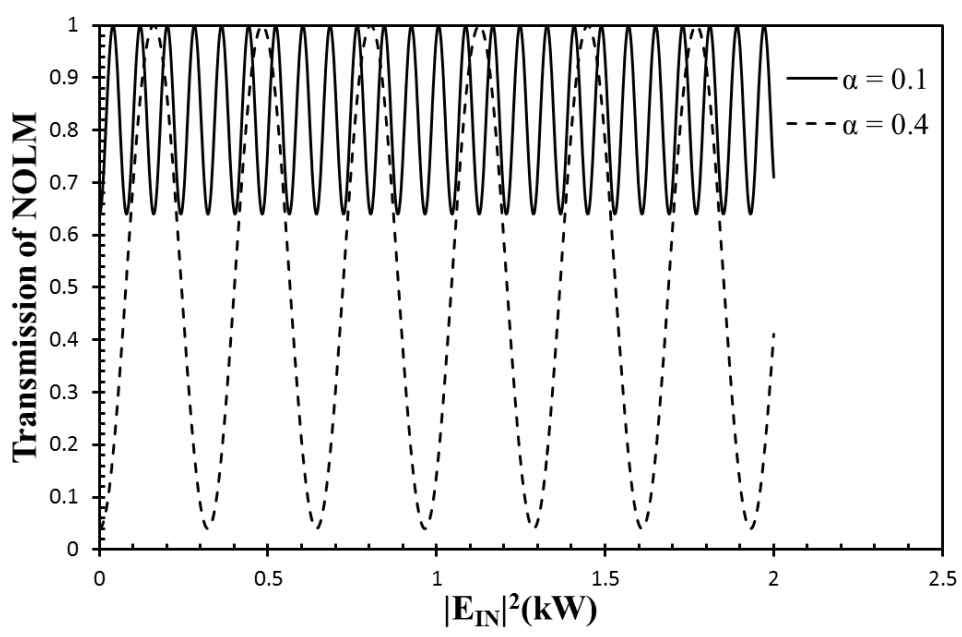

Fig. 2.3 Transmission of NOLM against input power in kilowatt.

As for NALM, an amplifier is added inside the loop. If the gain fiber is not in the center of the loop, light propagating clockwise and counter-clockwise will suffer different SPM, because the optical paths by the amplified light are different. Thus, the corresponding transmission of NALM is more complicated than that of NOLM, but the basic concept is the same. Assuming the gain is $g$ and the gain fiber is placed at the position close to clockwise direction, the phase difference is now also dependent on the gain. Now the output at port 2 can be written as: 


$$
\left|E_{02}\right|^{2}=\left|E_{I N}\right|^{2} \times g\left(1-2 \alpha(1-\alpha)\left\{1+\cos \left[(1-(g+1) \alpha)\left|E_{I N}\right|^{2} \times 2 \pi n_{2} L / \lambda\right]\right\}\right)
$$

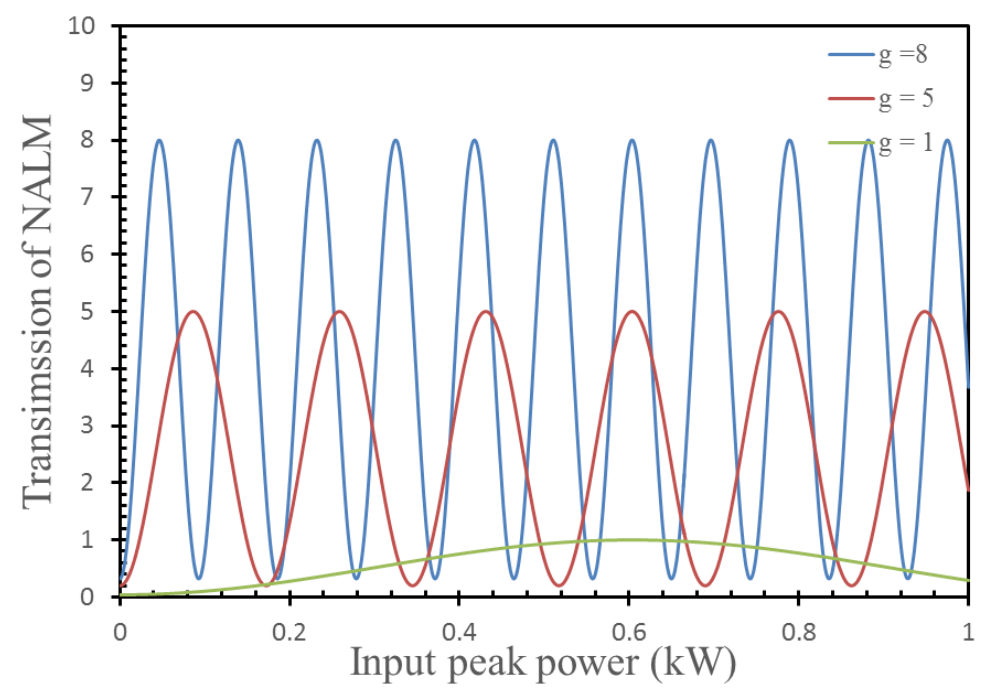

Fig. 2.4 Transmission of NALM against input power in kilowatt.

Fig. 2.4 shows transmission of an NALM with different gains as a function of input power in kilowatt. The simulation parameters are set as $\mathrm{n}_{2}=3.2 \times 10^{-16} \mathrm{~cm}^{2}$ $/ \mathrm{W}$, an effective area of $30 \mu \mathrm{m}^{2}$, loop length $\mathrm{L}=5 \mathrm{~m}$, coupler ratio $\alpha=0.4$, and $\lambda$ $=1.03 \mu \mathrm{m}$. All of them have a sinusoidal shape. Higher gain will lead to more peaks in certain range of input peak power. Compared to the transfer function of NOLM $(g=1)$, the one with NALM ( $g=8)$ has not only higher modulation depth, but also lower input peak power to reach the first maximum transmission peak, which means better self-starting ability.

\subsubsection{SESAM}

A semiconductor saturable absorber mirror (SESAM) is a mirror structure with an incorporated saturable absorber, which is made of semiconductor technology, such as GaAs, $\mathrm{Al}_{\mathrm{x}} \mathrm{Ga}_{1-\mathrm{x}} \mathrm{As}$ and $\mathrm{In}_{\mathrm{x}} \mathrm{Ga}_{1-\mathrm{x}} \mathrm{As}$. Such devices are mostly used for the generation of ultrafast pulses by passive mode locking of various types of lasers [64-66]. Typically, a SESAM contains a semiconductor Bragg mirror and a single quantum well absorber layer. The materials of the Bragg mirror have a larger bandgap energy, so that essentially no absorption occur in that region. Two 
important parameters of SESAM are modulation depth and saturation fluence. Higher modulation depth will lead to easier self-starting of the mode-locked pulse and saturation fluence determines the burning threshold of the SESAM.

\subsection{Gain medium}

Rare-earth doped silica fibers can provide required gain for amplification in a fiber laser. Thanks to the booming of optical communication, erbium-doped fiber amplifiers were extensively developed since they amplify light around the 1.55 $\mu \mathrm{m}$ wavelength region, where standard telecom fibers have minimum loss [67, 68]. In addition to this gain medium, fibers using other rare-earth ions such as ytterbium and neodymium as dopants allow us to significantly extend the achievable spectral range to around $1 \mu \mathrm{m}$, and to achieve higher power levels. The fiber laser presented in this dissertation uses ytterbium doped fibers as the gain medium.

Yb-doped silica fibers are of particular significance for the following three reasons. First, Ytterbium has a high absorption peak at 976 nm, as shown in Fig. 2.5. This wavelength coincides with the pumping diodes at $976 \mathrm{~nm}$, which have primarily been well developed for pumping Er-doped fiber amplifier in the telecommunication industry. Secondly, Ytterbium has a high quantum efficiency ( $95 \%)$, as the lasing band (highest emission peak is around $1030 \mathrm{~nm}$ ) is very close to the pump wavelength $(976 \mathrm{~nm})$. Together with the reason that very high doping concentrations are possible in ytterbium doped fibers, high slope efficiencies up to $\sim 80 \%$ could be achieved. Besides, high doping concentrations allow for very high gain in a short length of optical fiber, thus avoiding unwanted nonlinear effects. Last and most importantly, Ytterbium has a broad gain bandwidth, offering an almost ideal gain medium for the generation of ultrafast fiber laser since the spectral width of femtosecond or picosecond fiber lasers can be tens of nanometers. Ultrashort pulse generation in an $\mathrm{Yb}$-doped fiber laser was demonstrated $[69,70]$, and shortly after that configurations for higher energy pulses [71] and wavelength tunability were achieved [64]. 


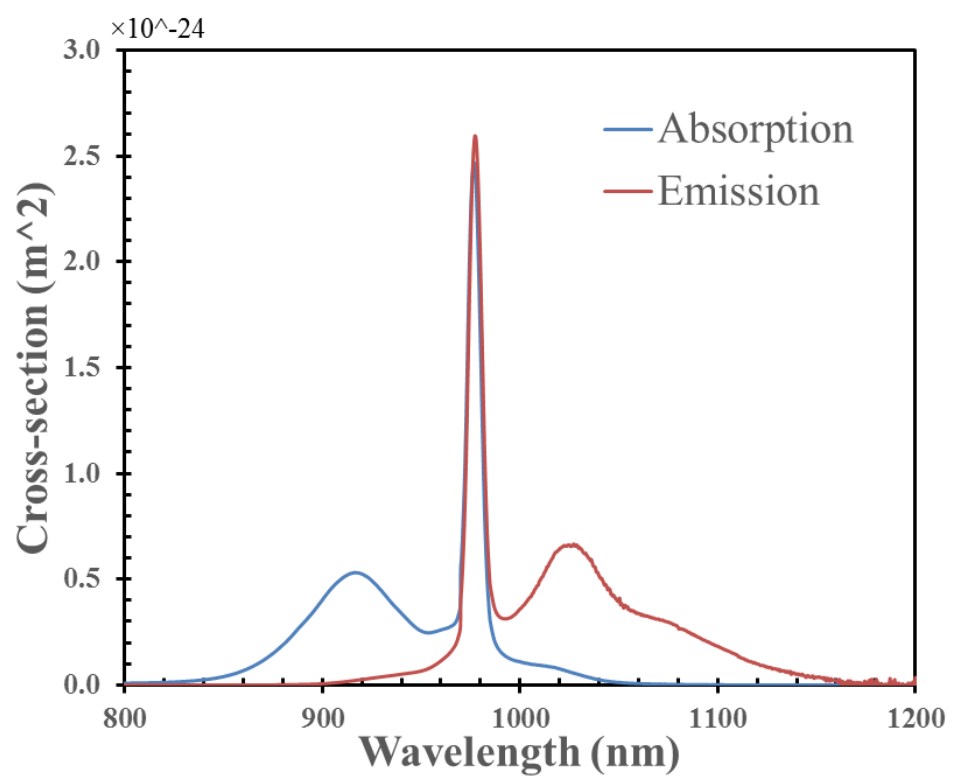

Fig. 2.5 Absorption and emission cross-section for $\mathrm{Yb}^{3+}$ - doped silica fiber.

\subsection{Cavity loss}

There are mainly three types of losses in this DS fiber laser, which includes fiber losses, splicing losses and the losses induced by output coupler. The intrinsic losses of a silica fiber is very low, thanks to the rapid development of fiber communication. A typical loss is are $0.2 \mathrm{~dB} / \mathrm{km}$ near $1.55 \mu \mathrm{m}$ and $1 \mathrm{~dB} / \mathrm{km}$ around $1 \mu \mathrm{m}$, which can almost be neglected. The splicing loss is controlled to be less than $0.05 \mathrm{~dB}$ for each splicing point. There are around 10 splicing points in this configuration. Thus, the total splicing loss is less than $0.5 \mathrm{~dB}$. The main loss, which helps to build the DS mechanism, comes from output coupler. Different output coupler ratios, like 30\% (1.55 dB loss), 70\% (5.23 dB loss), 80\% (6.99 dB loss), have been employed in this laser configuration.

\subsection{Dispersion and nonlinearity}

The reason why there are dispersion and nonlinearity when light propagates inside the silica fiber is that the refractive index is frequency and intensity dependent, instead of being a constant. Chromatic dispersion manifests through the frequency 
dependence of the refractive index $n(\omega)$, while nonlinearity corresponds to the intensity dependence of the refractive index $n(I)$.

\subsubsection{Chromatic dispersion}

Essentially speaking, the origin of chromatic dispersion is due to the characteristic resonance frequencies at which the medium absorbs the electromagnetic radiation through oscillations of bound electrons. Far from the medium resonances, the refractive index is well approximated by the Sellmeier equation:

$$
n^{2}(\omega)=1+\sum_{j=1}^{m} \frac{B_{j} \omega_{j}^{2}}{\omega_{j}^{2}-\omega^{2}}
$$

where $\omega_{j}$ is the resonance frequency and $B_{j}$ is the strength of $j$ th resonance. Both of them is associated with constituents of optical fibers. Fiber dispersion plays a critical role in propagation of short optical pulses because different spectral components associated with the pulse travel at different speeds given by $\mathrm{c} / \mathrm{n}(\omega)$. Mathematically, the effects of fiber dispersion are accounted for by expanding the mode propagation constant $\beta$ in a Taylor series about the frequency $\omega_{0}$ at which the pulse spectrum is centered:

$$
\beta(\omega)=n(\omega) \frac{\omega}{c}=\beta_{0}+\beta_{1}\left(\omega-\omega_{0}\right)+\frac{1}{2} \beta_{2}\left(\omega-\omega_{0}\right)^{2}+\ldots
$$

in which

$$
\beta_{m}=\left(\frac{d^{m} \beta}{d \omega^{m}}\right)_{\omega=\omega_{0}} \quad(\mathrm{~m}=0,1,2 \ldots)
$$

$\beta_{1}$ and $\beta_{2}$ is essentially important and they can be expressed as:

$$
\beta_{1}=\frac{1}{v_{g}}=\frac{n_{g}}{c}=\frac{1}{c}\left(n+\omega \frac{d n}{d \omega}\right)
$$




$$
\beta_{2}=\frac{1}{c}\left(2 \frac{d n}{d \omega}+\omega \frac{d^{2} n}{d \omega^{2}}\right)
$$

where $\mathrm{n}_{\mathrm{g}}$ is group index and $\mathrm{v}_{\mathrm{g}}$ is group velocity. The envelope of an optical pulse moves with group velocity while the parameter $\beta_{2}$ stands for dispersion of the group velocity and is responsible for pulse broadening, which is so-called groupvelocity dispersion (GVD), and $\beta_{2}$ is called GVD parameter. Fig. 2.6 gives the changing of group index over wavelength for a typical single-mode-fiber. There is an interesting wavelength at $1.27 \mu \mathrm{m}$ where the variation of $\mathrm{n}_{\mathrm{g}}$, or $\beta_{2}$, equals to zero. This wavelength is referred to as the zero-dispersion wavelength and is denoted as $\lambda_{D}$. If the wavelength is smaller than $\lambda_{D}, \beta_{2}$ is positive and the fiber is said to exhibit normal dispersion. In the normal-dispersion regime, low-frequency (red-shifted) components of an optical pulse travel faster than high-frequency (blue-shifted) components of the same pulse. In contrary, in anomalous-dispersion regime where $\beta_{2}$ is negative $\left(\lambda>\lambda_{D}\right)$, low-frequency (red-shifted) components of an optical pulse travel slower than high-frequency (blue-shifted) components of the same pulse.

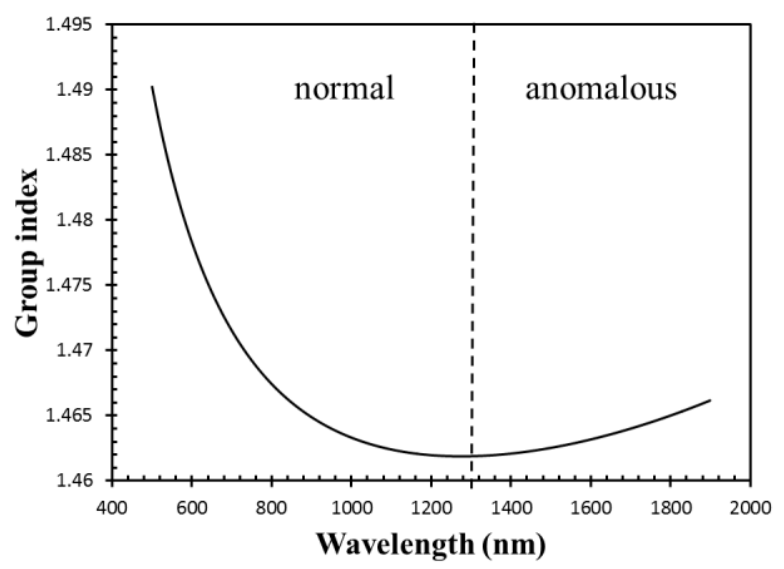

Fig. 2.6 Variation of group index $\mathrm{n}_{\mathrm{g}}$ with wavelength for fused silica.

For the generation of conventional solitons, wavelength at anomalous-dispersion regime is of great interest, because the negative chirp generated by anomalousdispersion is balanced by the positive chirp generated by nonlinearity (SPM) and thus the pulse is prevented from broadening. 


\subsubsection{Nonlinearity}

The origin of nonlinear response is due to anharmonic motion of bound electrons under the influence of an applied field. Most of the nonlinear effects in optical fibers is expressed by the intensity dependence of the refractive index, which can be written as:

$$
n^{\prime}\left(\omega,|E|^{2}\right)=n(\omega)+n_{2}|E|^{2}
$$

where $n(\omega)$ is the linear part given by Eq. (2.6), $|E|^{2}$ is the optical intensity inside the fiber, and $\mathrm{n}_{2}$ is the nonlinear-index coefficient, which is related to material property. The intensity dependence of the refractive index leads to a large number of interesting nonlinear effects, in which the two most widely studied are selfphase modulation (SPM) and cross-phase modulation (XPM). SPM refers to the self-induced phase shift experienced by an optical field during its propagation in optical fibers. The magnitude of SPM is related to the phase induced by nonlinear-index.

$$
\phi^{\prime}=n^{\prime} k_{0} z=\phi+\phi_{N L}=\left(n+n_{2}|E|^{2}\right) k_{0} z
$$

where $\mathrm{k}_{0}=2 \pi / \lambda$ and $\mathrm{z}$ is the fiber length. The intensity-dependent nonlinear phase shift $\phi_{\mathrm{NL}}$ is caused by SPM. Consider a plane wave pulse propagating in the $\mathrm{z}$ direction. Its electric field could be written as:

$$
E=\frac{1}{2} E_{0}(z, t) \exp \left(-j n k_{0} z\right) \exp \left(j \omega_{0} t\right)
$$

Substituting Eq. 2.12 into Eq. 2.13, we can get:

$$
E=\frac{1}{2} E_{0}(z, t) \exp \left(-j n_{0} k_{0} z\right) \exp \left(j \omega_{0} t-j \delta \phi(t)\right)
$$

in which 


$$
\delta \phi(t)=n_{2}\left|E_{0}(z, t)\right|^{2} k_{0} z
$$

The additional time-varying phase term has the effect of introducing new frequency components to the pulse. The new frequency could be derived by differentiating the time-varying terms in the exponent:

$$
\omega^{\prime}=\frac{\partial}{\partial t}\left(\omega_{0} t-\delta \phi(t)\right)=\omega_{0}-\delta \omega(t)
$$

in which

$$
\delta \omega(t)=-\frac{\partial}{\partial t} \delta \phi(t)=-n_{2} k_{0} z \frac{\partial}{\partial t}\left|E_{0}(z, t)\right|^{2}
$$
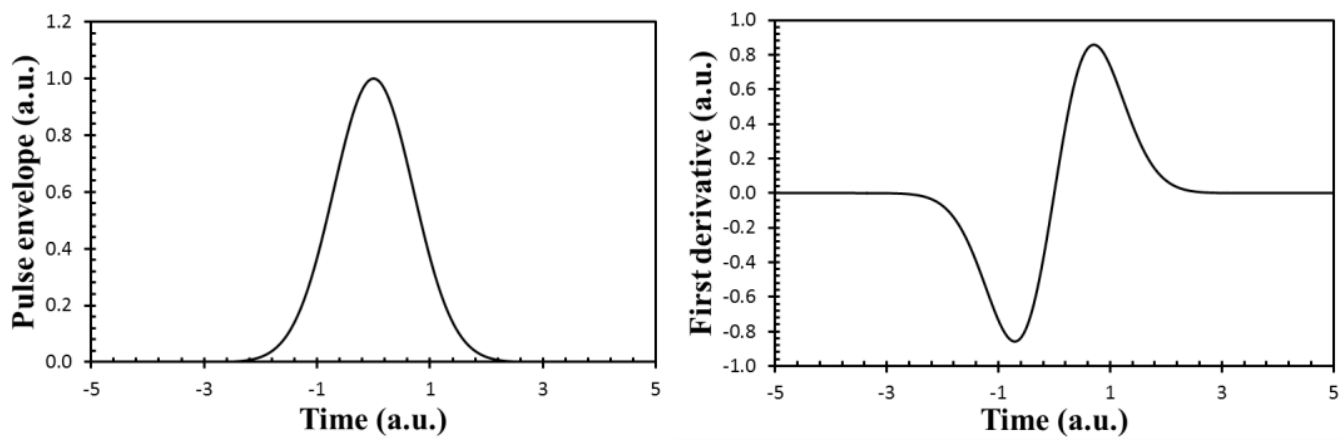

Fig. 2.7 Plots of a Gaussian pulse intensity envelope (left) and corresponding chirp raised by SPM (right).

Assume we have a Gaussian pulse propagating inside a silica fiber. According to Eq. 2.17, first derivative of the pulse envelope gives the frequency offset. As shown in Fig. 2.7, SPM will induce a positive and nearly linear chirp in the middle of the Gaussian pulse. If the laser pulse wavelength is at anomalous dispersion regime, which will induce negative linear chirp, the total chirp of the pulse will be canceled and the so-called soliton could maintain its shape and pulse width when propagating through fibers. However, if the wavelength is at normal dispersion regime, which will cause positive linear chirp, the joint action of the SPM and dispersion on the chirp cannot be canceled and thus an extra dissipative module needs to be included into the all-normal-dispersion laser system to cut 
down the ever-enlarging pulse width. That is why a spectral filter is an essential component for the ANDi fiber lasers.

\subsection{Spectral filter}

As mentioned in the previous section, the dominant pulse-shaping mechanism of ANDi fiber lasers is filtering of a highly chirped pulse in the cavity. Because of the linear chirp induced by normal dispersion and SPM, the wavelength is linearly increased throughout the pulse. Therefore, the filtering of the spectral domain also leads to the filter in the time domain. This dissipative process will cut off the ever-enlarging pulse width in the ANDi fiber lasers and help the DS pulses reach a stable state, which results from the composite balance between spectral filtering on one side, and nonlinearity, dispersion and gain on the other side. The $3 \mathrm{~dB}$ bandwidth of the filter plays an important role in the forming of dissipative solitons. Too small or too large will both break the balance [72]. The proper way of designing a DS fiber laser is to fix the $3 \mathrm{~dB}$ bandwidth of the filter, and then design all other parameters of the lasers, such as cavity length, output coupler ratio and saturated absorber according to the bandwidth in order to make the system stable.

\subsection{Summary}

In this chapter, several physical modules that determine the forming of a stable dissipative soliton (DS), including gain medium, output coupler, dispersion, nonlinearity, band-pass filter and stature absorber have been introduced. Each

module is essential and plays certain roles in the generation of DS. In chapter 3, a numerical model for DS mode-locked fiber laser simulations is developed based on these physical modules. 


\section{Chapter 3}

\section{Simulation of DS fiber lasers}

A numerical model for DS mode-locked fiber laser is presented in this chapter. The model is based on the nonlinear Schrodinger equation which has been described in chapter 2. Several parameters can influence the performance of DS laser including nonlinear phase shift accumulated by the pulse propagation, spectral filter bandwidth, and group-velocity dispersion etc. Trends in the performance as these parameters varying are studied. In the simulations, a pulse is generated from noise over many round trips times until a stable solution is reached.

\subsection{Nonlinear Schrodinger equation}

The pulse propagation within an ANDi laser is modeled by the appropriate terms of the following revised NLS equation [58-60]:

$$
\frac{\partial A(z, \tau)}{\partial z}=-i \frac{\beta_{2}}{2} \frac{\partial^{2} A(z, \tau)}{\partial \tau^{2}}+i \gamma|A(z, \tau)|^{2} A(z, \tau)-\frac{1}{2}(g-\alpha) A(z, \tau)+\frac{g T_{2}^{2}}{2} \frac{\partial^{2} A(z, \tau)}{\partial \tau^{2}}
$$

where $\mathrm{A}(\mathrm{Z}, \tau)$ is the electric field envelope, while $\mathrm{z}$ is the propagation distance and $\tau$ is measured in a frame of reference moving with the pulse at the group velocity $v_{g}\left(\tau=t-z / v_{g}\right)$. The first two terms on the right side represent group velocity dispersion (GVD) and self-phase modulation (SPM) respectively. The third one is gain minus loss, while the last one is gain dispersion. The last two terms should be taken into account when the pulse duration is larger than 100fs. $\mathrm{g}$ is the net gain function for the Yb-doped gain fiber, which is modeled according to 


$$
g=\frac{g_{0}}{1+E_{p u l s e} / E_{\text {sat }}}
$$

where $\mathrm{g}_{0}$ is the small-signal gain. A Gaussian gain shape with $45 \mathrm{~nm}$ bandwidth is assumed. The gain saturation energy $\mathrm{E}_{\text {sat }}$ is set according to the pump power. In the simulation, $E_{\text {sat }}$ is varied from 1 to $6 \mathrm{~nJ} \mathrm{~T}_{2}$ is the dipole relaxation time, which can be written as

$$
T_{2}=\frac{2}{\Delta \omega_{g}}=\frac{2}{2 \pi \Delta f}=\frac{\lambda^{2}}{\pi c} \frac{1}{\Delta \lambda}
$$

The simulation is based on the experimental setup shown in Fig. 3.1. The ring cavity is constructed with one main loop and one NALM loop. The main loop consists of a 980/1040 nm wavelength division multiplexer (WDM \#1), a $50 \mathrm{~cm}$ long high-concentration Yb-doped PM fiber with an estimated core absorption of $750 \mathrm{~dB} / \mathrm{m}$ at $976 \mathrm{~nm}$, a segment of standard PM 980 single-mode fiber, a PM isolator, a 30/70\% PM output coupler with the $70 \%$ transmission port as an output and a narrow bandpass filter centered at $1030 \mathrm{~nm}$ with a bandwidth of $2.76 \mathrm{~nm}$. The NALM loop is connected to the main loop by a 40/60 PM coupler between the isolator and the output coupler. The loop consists of a $30 \mathrm{~cm}$-long highconcentration Yb-doped PM fiber, a 980/1040 nm WDM (\#2), and a segment of PM980 single mode fiber $(2 \mathrm{~m})$. The NALM loop was used as an equivalent SA whose transfer function of NALM is described in Eq. 2. 5.

The band-pass filter can be described by the transfer function following a Gaussian profile as

$$
H(f)=\alpha_{f} \exp \left[\ln (\sqrt{2}) \times\left(\frac{f}{\Delta f_{3 d B}}\right)^{2}\right]
$$

where $\alpha_{\mathrm{f}}$ is the insertion loss and $\Delta \mathrm{f}_{3 \mathrm{~dB}}$ is $3 \mathrm{~dB}$ bandwidth of the filter.

Split-step Fourier transform method has been applied to solve the NLSE, which 
has been proved to be one of the powerful methods due to its easy implementation and speed compared to other methods [75].

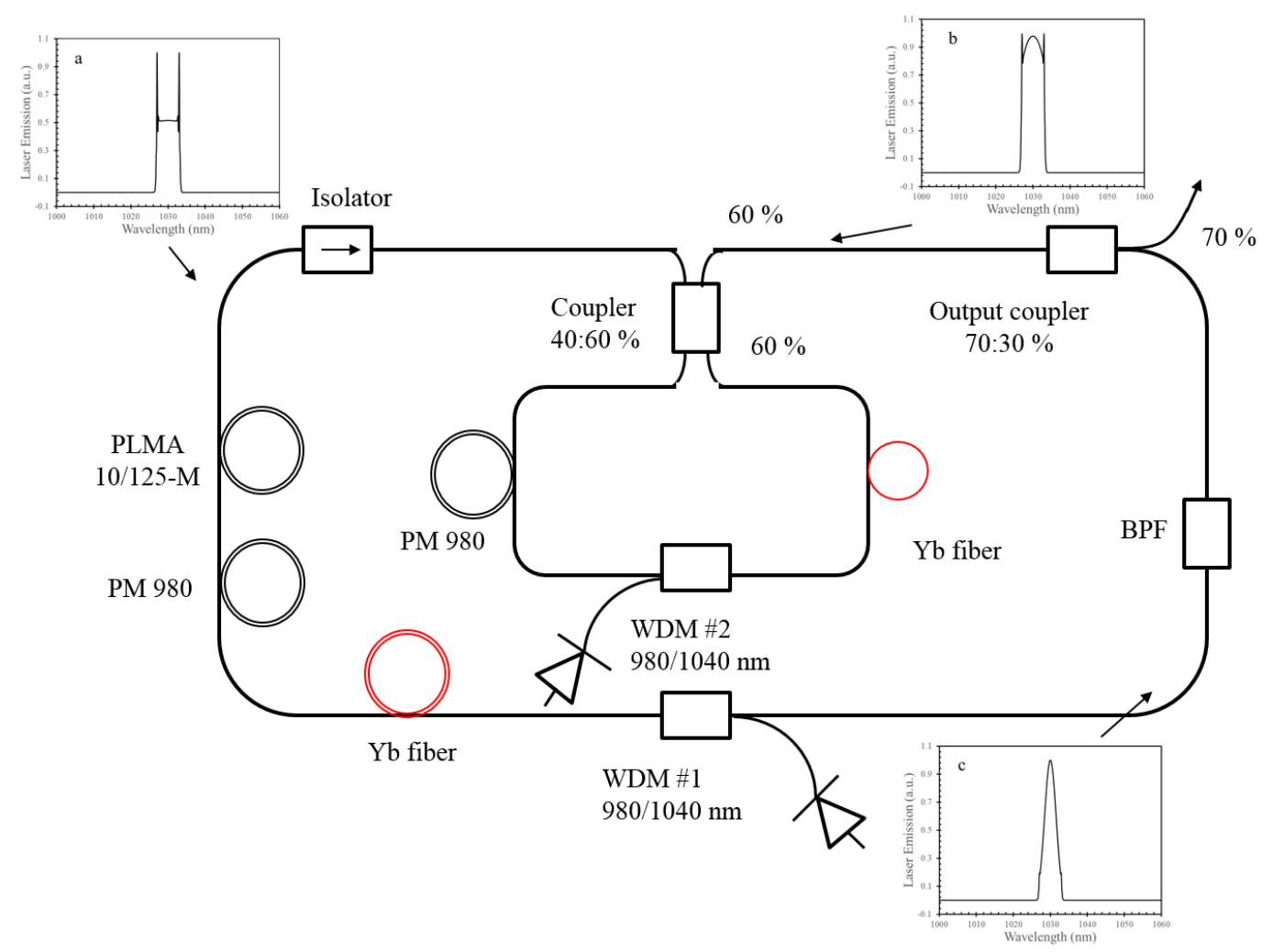

Fig. 3.1 Schematic of Yb all-fiber NALM-based ANDi fiber laser and typical numerical simulation result: (a) after the long extra PM fibers, (b) after the NALM, (c) after the filter.

\subsection{Cavity parameters}

Table 3.1 Fiber Parameters for DS laser simulation

\begin{tabular}{cccccc}
\hline & $\beta_{2}\left(\mathrm{ps}^{2} / \mathrm{m}\right)$ & $\gamma\left(\mathrm{m}^{-1} \mathrm{~W}^{-1}\right)$ & $\mathrm{L}(\mathrm{m})$ & $\mathrm{E}_{\mathrm{sat}}(\mathrm{pJ})$ & $\mathrm{g}_{0}(\mathrm{~dB} / \mathrm{m})$ \\
\hline PM980_1 & 0.0024 & 0.0049 & 80 & 0 & 0 \\
YDF & 0.0024 & 0.0049 & 0.5 & 1000 & 16 \\
PLMA10/125 & 0.0016 & 0.0012 & 50 & 0 & 0 \\
\hline
\end{tabular}


Table 3.2 Other Parameters for DS laser simulation

\begin{tabular}{|c|c|c|c|}
\hline \multirow{2}{*}{ YDF } & Center Wavelength & \multicolumn{2}{|c|}{ Gain Bandwidth } \\
\hline & $1030 \mathrm{~nm}$ & \multicolumn{2}{|c|}{$45 \mathrm{~nm}$} \\
\hline \multirow{2}{*}{ SF } & Center Wavelength & \multicolumn{2}{|c|}{ 3dB Bandwidth } \\
\hline & $1030 \mathrm{~nm}$ & \multicolumn{2}{|c|}{$2.7 \mathrm{~nm}$} \\
\hline \multirow{2}{*}{ NALM } & Gain & Loop length & ratio \\
\hline & 5 & $4 \mathrm{~m}$ & $60: 40$ \\
\hline \multirow{2}{*}{$\mathrm{OC}$} & \multicolumn{3}{|c|}{ Output Coupler Coefficient } \\
\hline & \multicolumn{3}{|c|}{$70 \%$} \\
\hline
\end{tabular}

All the cavity parameters used in this simulation are listed in Table 3.1\&3.2. All these values are corresponding to our experimental configuration.

Figure 3.1 shows how the spectrum evolves in the main loop of the cavity. After the gain fiber amplifies the pulse, the enhanced peak power in the SMF induces a substantial nonlinear phase shift, which produces sharp peaks at the edges of the spectrum, so-called "cat-ears" (shown in Fig. 3.1a). Such sharp peaks become week after NALM loop (shown in Fig. 3.1b). After traversing the spectral filter, the spectrum shows a Gaussian-shaped top that follows the filter transmission curve (shown in Fig. 3.1c). The spectral filter, and to a lesser degree the saturable absorber, cut off the pulse wings and return the spectrum to its starting shape (shown in Fig. 3.1c).

The corresponding pulse width evolution is shown in Fig. 3.2. The pulse broadens after traveling through a long SMF fiber (because of the dispersion), and was trimmed down after the NALM and the spectral filter, because the pulse is highly chirped. The pulse width cutting ability of the filter (from 39 ps to $17 \mathrm{ps}$ ) is more 
effective than that of the NALM (from 51 ps to 39 ps).

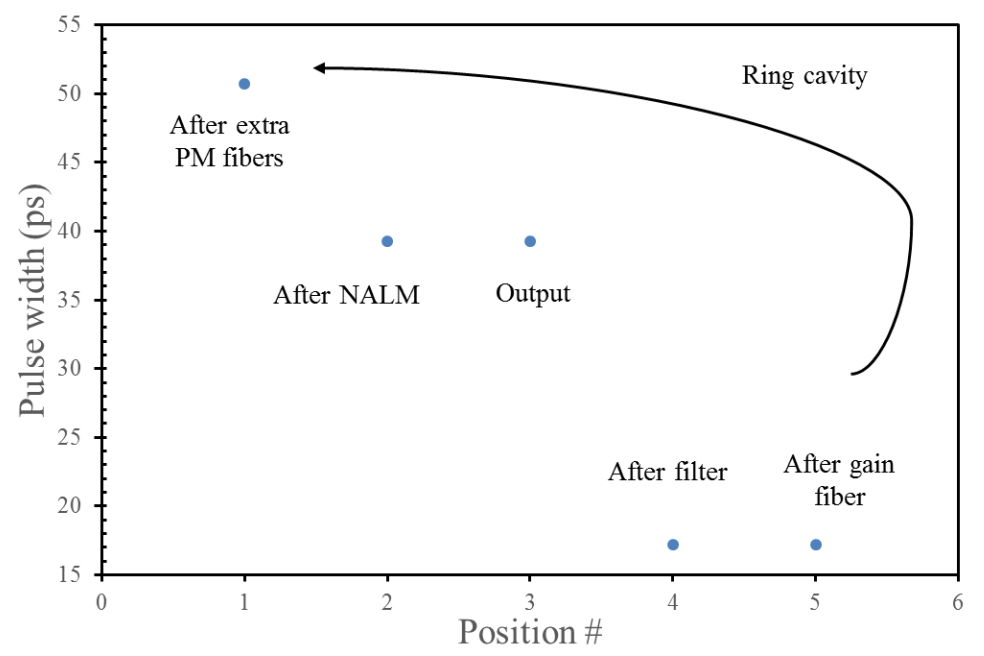

Fig. 3.2 Pulse duration evolution of the NALM-based ANDi fiber laser.

\subsection{Variation of laser parameters}

\subsubsection{Cavity length}

The first design parameter changed here is the cavity length. By changing the length of the extra PM980 fiber, the GVD in the laser will change correspondingly. Fig 3.3 shows two simulated laser emission spectra at two different length of extra PM 980 segment, $30 \mathrm{~m}$ and $150 \mathrm{~m}$, respectively. Other parameters are the same as described in Table $3.1 \& 3.2$.

From the simulation results as shown in Fig. 3.3, stable mode-locking can be achieved with both $30 \mathrm{~m}$ and $150 \mathrm{~m}$ extra PM 980 fiber. Further increasing the fiber length to over $150 \mathrm{~m}$ or decreasing the fiber length to less than $30 \mathrm{~m}$ will both lead to noise-like pulses. Such results were verified by the later experimental observations. When the extra PM 980 fiber is $30 \mathrm{~m}$ long, the laser spectrum shows a typical "cat ears" shape (Fig. 3.3(a)), which is the result of severe SPM. The corresponding temporal pulse has a "ring-bell" shape as shown in Fig. 3.3(b). When the extra PM 980 fiber is $150 \mathrm{~m}$ long, the longer cavity can stretch the pulse more effectively, thus maintaining low peak power inside the cavity, which 
will reduce the effect of SPM. Therefore, the corresponding spectrum has a narrower bandwidth and two steep edges as shown in Fig. 3.3(c). The pulse shape at this condition is more like a Sech function as shown in Fig. 3.3(d).
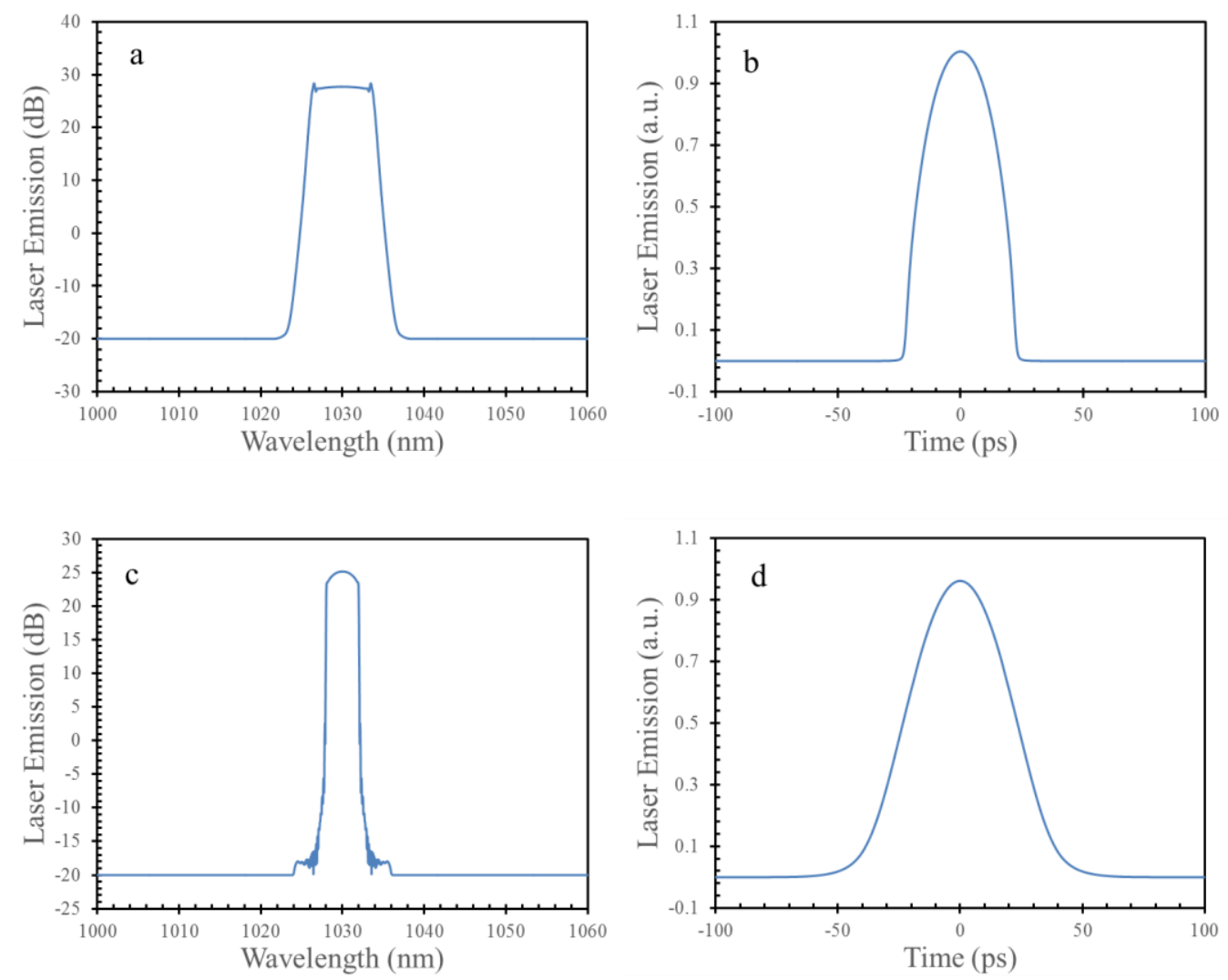

Fig. 3.3 Output spectrum and pulse shape with 30 m extra PM 980 fiber (a, b); with 150 m extra PM 980 fiber $(c, d)$.

The dependence of the laser output parameters, namely pulse width, pulse energy and peak power on the cavity length is summarized in Fig.3.4. The output ratio and filter bandwidth is fixed at $70 \%$ and $2.7 \mathrm{~nm}$ for all the simulation in this section. The pulse width increases linearly [Fig 3.4(a)] with the increasing of the cavity length due to the dispersion. The pulse energy and peak power also increases [Fig 3.4(b, c)] because of lower reputation rate with a longer cavity length. Therefore, a long cavity length is favorable for high pulse energy generation and a short cavity length has the advantage of narrow pulse width, which can be easier to de-chirp to obtain femtosecond output. 

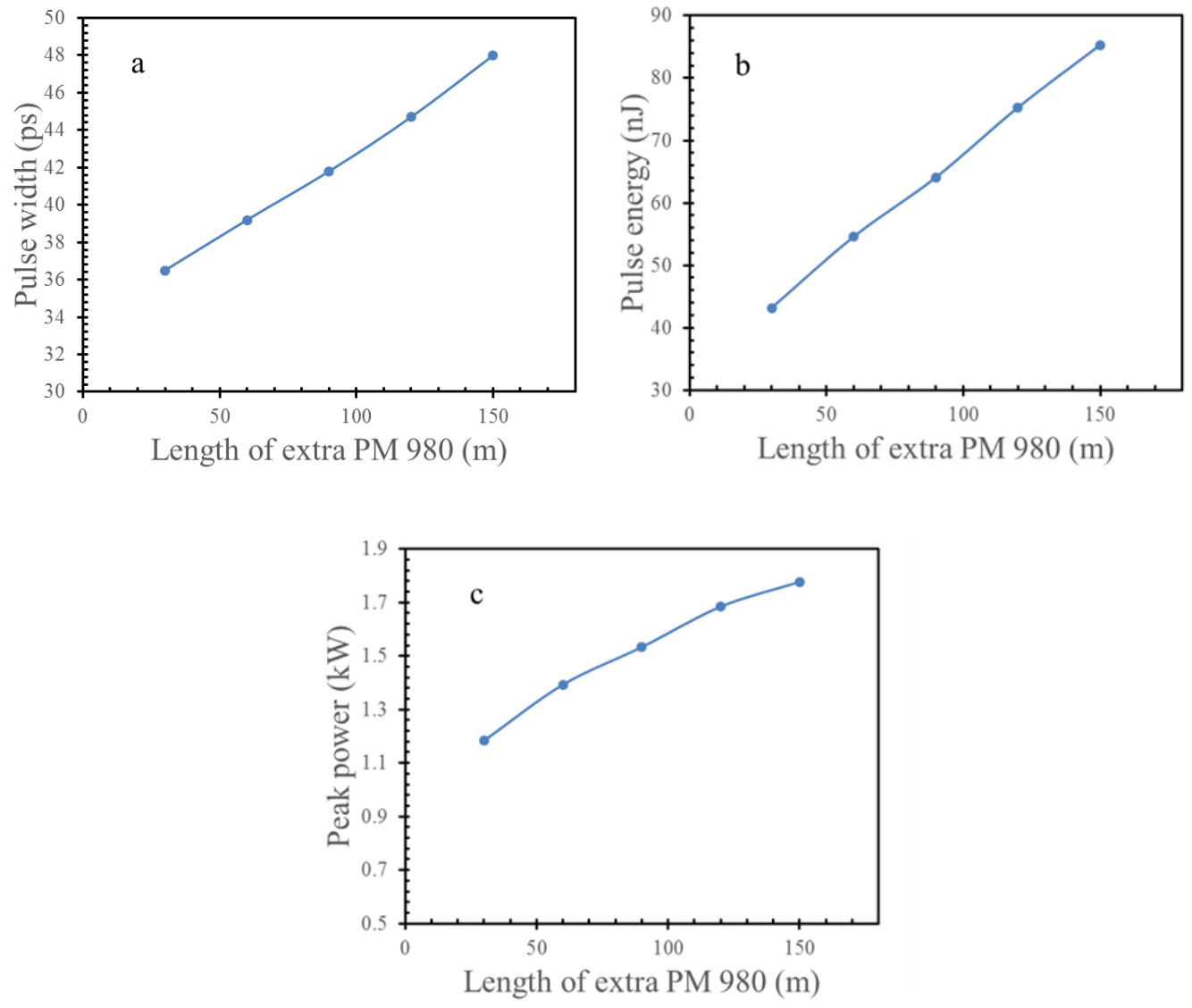

Fig. 3.4 Laser performance versus length of extra PM 980 fiber: (a) pulse width, (b) pulse energy, (c) peak power.

\subsubsection{Output coupler ratio}

The second parameter changed here is the output coupler ratio. Figure 3.5 shows two simulation results with two different output coupler ratios, $50 \%$ and $80 \%$ respectively. Other parameters are the same as described in Table 3.1\&3.2. 

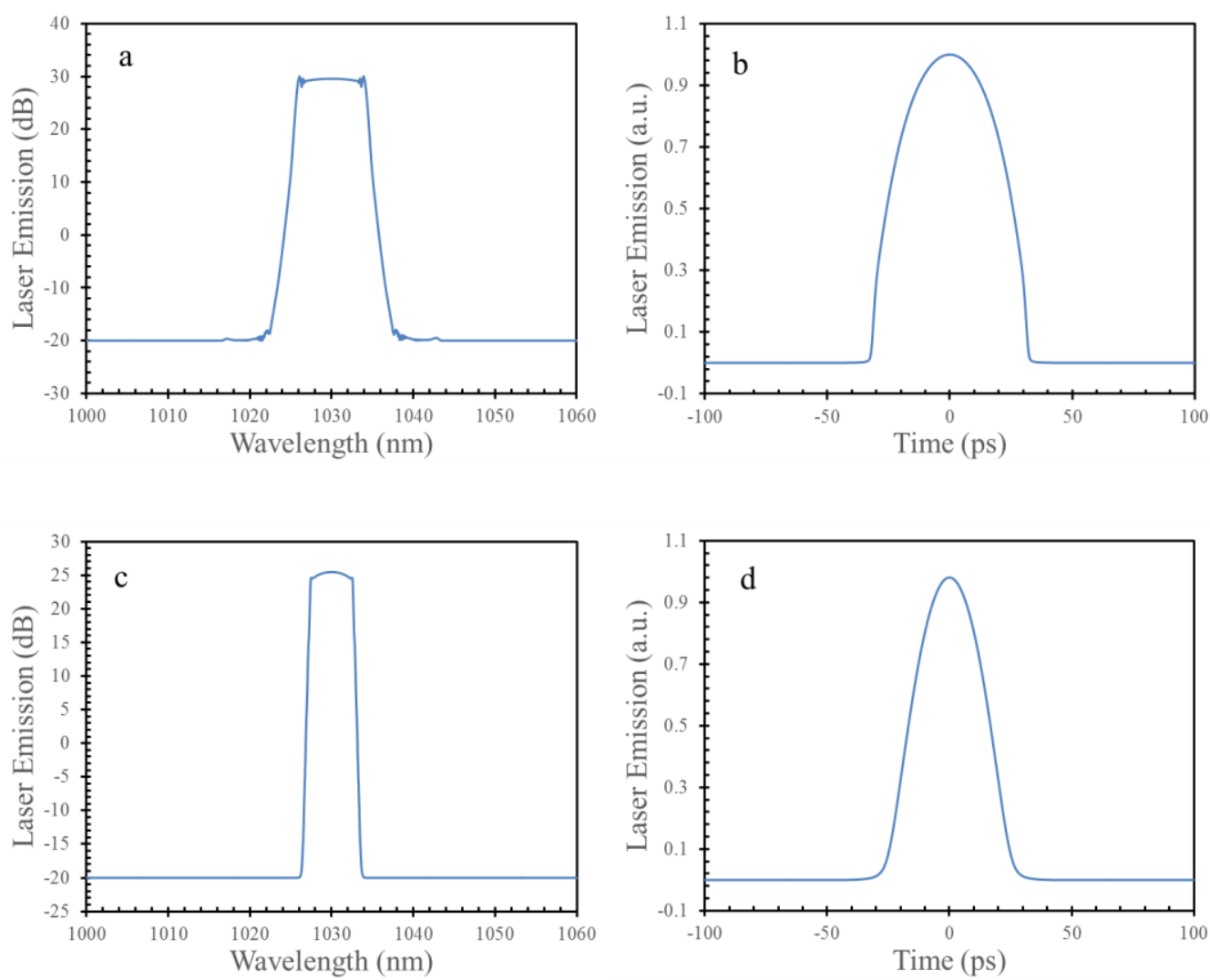

Fig. 3.5 Output spectrum and pulse shape with $50 \%$ power output (a, b); with $80 \%$ power output (c, d).

The laser can produce DS pulses when the output ratio is between $50 \%$ and $80 \%$. When the output ratio is at $50 \%$, the laser spectrum shows two typical "cat ears" shape (Fig. 3.5(a)), which is resulted from the severe SPM. The corresponding temporal pulse has a "ring-bell" shape as shown in Fig. 3.5(b). When the output ratio increases to $80 \%$, less power is left in the cavity, thus it can maintain low peak power inside, which will reduce the effect of SPM. Therefore, the corresponding spectrum has narrower bandwidth and two steep edges [Fig. 3.5(c)]. The pulse shape at this condition is more like Sech function as shown in Fig. 3.5(d). Smaller output coupler ratio will lead to larger amount of power remaining in the cavity, which means larger nonlinear phase shifts. ANDi modelocking is easier to obtain with a small nonlinear phase shift. That is why a large output coupler ratio is favored in the ANDi lasers. 
The dependence of the laser output parameters on the output coupler ratio is summarized in Fig.3.6. The extra PM fiber length and filter bandwidth is fixed at $60 \mathrm{~m}$ and $2.7 \mathrm{~nm}$ for all the simulation in this section. The pulse energy will increase almost linearly with the increase of output ratio as expected [Fig 3.6(b)]. Meanwhile, the pulse width will decrease when the output ratio rises [Fig 3.6(a)] due to less SPM inside the cavity. Therefore, the corresponding peak power can increase tremendously with the output ratio increasing [Fig 3.6(c)]. Thus, high output power ratio is preferred in the design of such ANDi lasers.
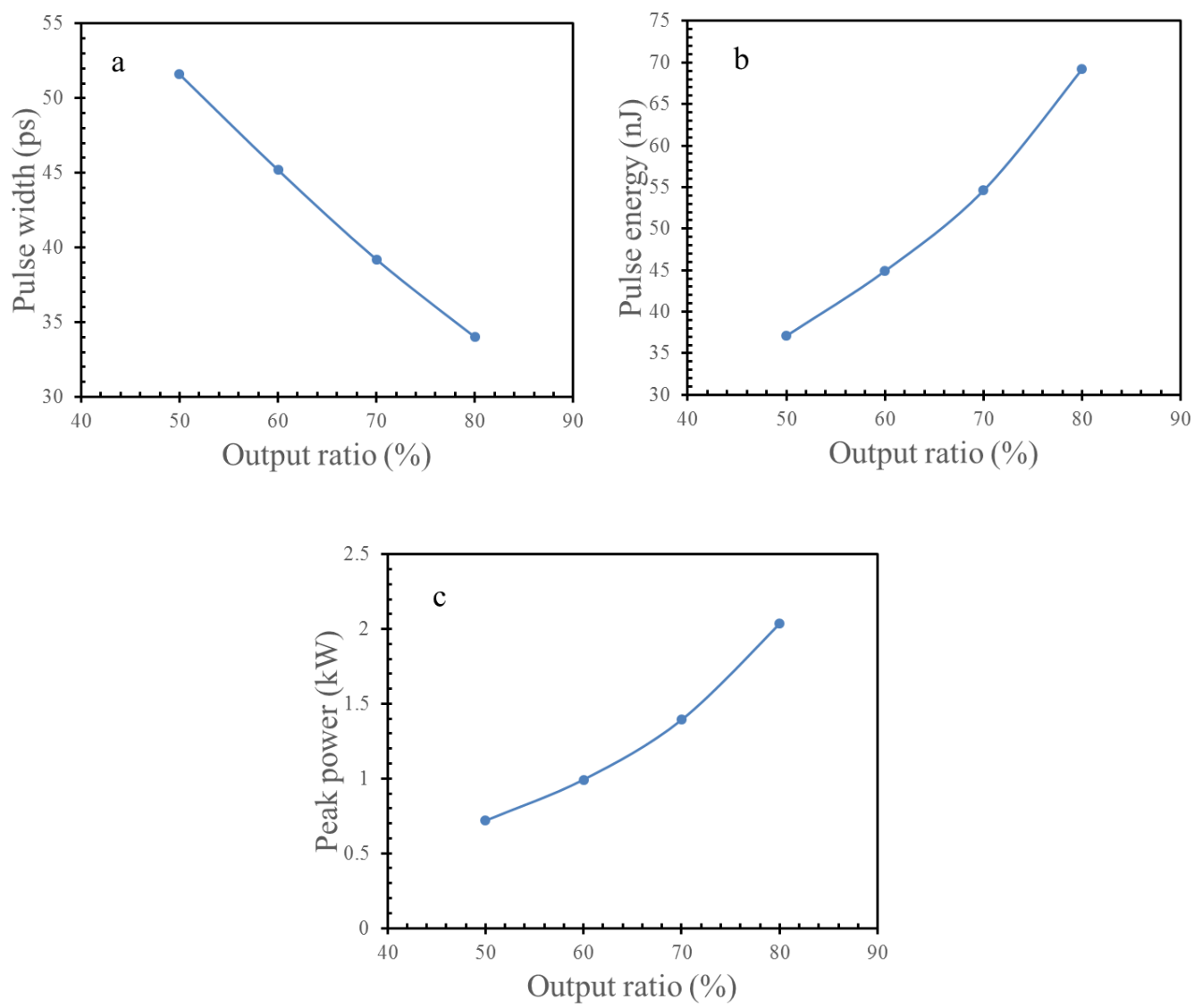

Fig. 3.6 Laser performance versus output ratio: (a) pulse width, (b) pulse energy, (c) peak power. 


\subsubsection{Spectral filter bandwidth}
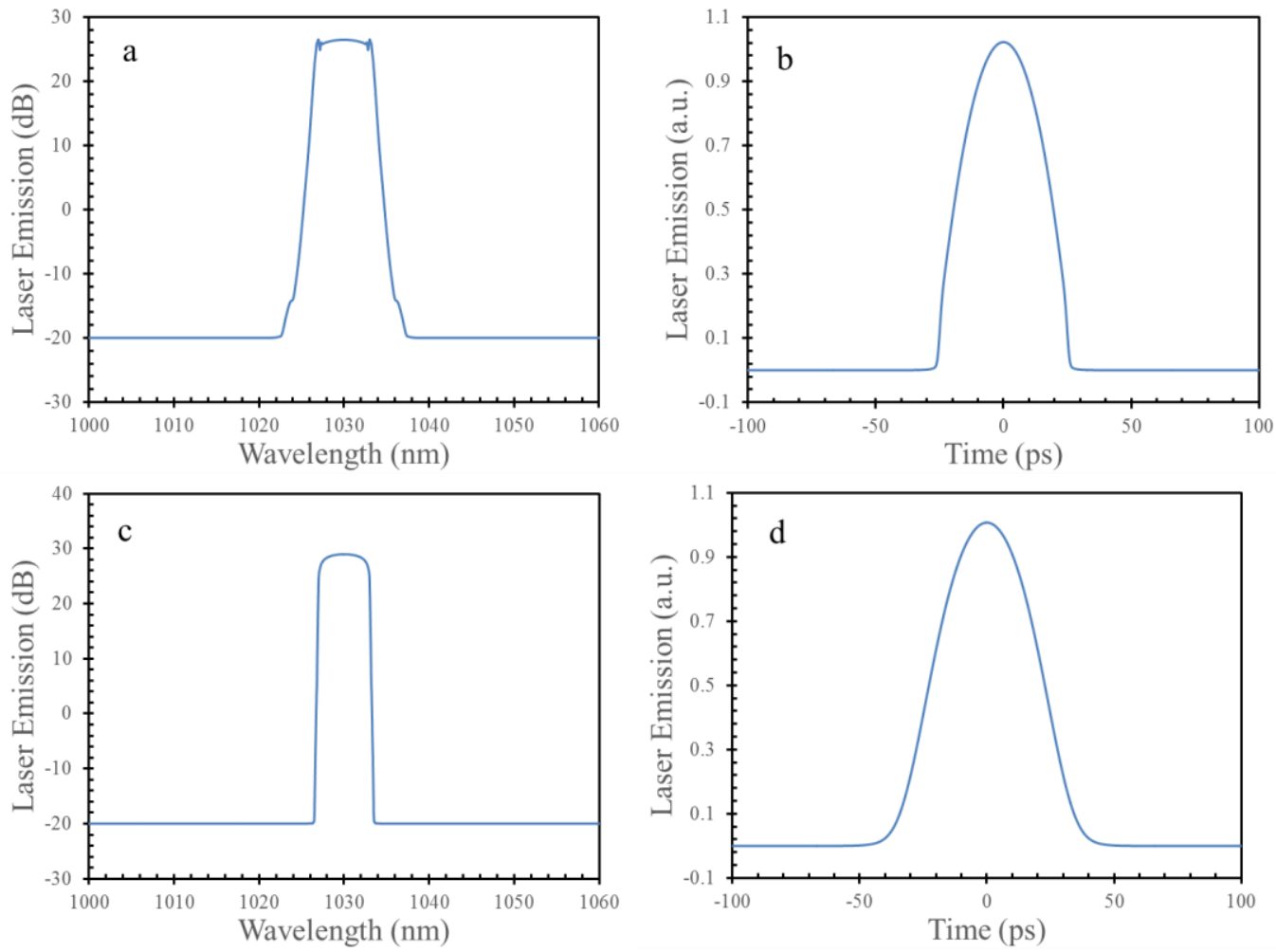

Fig. 3.7 Output spectrum and pulse shape with different filter bandwidth, with $2.2 \mathrm{~nm}$ (a, b) and $4.2 \mathrm{~nm}(\mathrm{c}, \mathrm{d})$.

The next laser parameter to be investigated is the effects of the spectral filter bandwidth. Figure 3.7 shows two simulation results with different filters' BW, 2.2 $\mathrm{nm}$ and $4.2 \mathrm{~nm}$ respectively. Other parameters are the same as described in Table 3.1\&3.2. Smaller filter bandwidth leads to higher cutting capacity both in time and frequency domain, which is very effective in the control of nonlinear phase shifts. Thus, the output pulse with a small filter BW [Fig. (3.7a)] will have broader spectral width compared to the one with large filter BW [Fig. (3.7c)].

The dependence of the laser output parameters on filter bandwidth is summarized in Fig.3.8. The output ratio and extra PM 980 fiber length is fixed at $70 \%$ and 60 $\mathrm{m}$ for all the simulation in this section. Small filter BW is more effective to the pulse width cutting [Fig 3.8(a)], thus the output energy and peak power is also smaller compared to those with larger filter BW [Fig 3.8(b, c)]. Therefore, 
broader filter BW is favored in the design of high energy ANDi lasers. However, the filter BW cannot be larger than $4.2 \mathrm{~nm}$ in this simulation, otherwise the cavity will start to produce noise-like pulses.
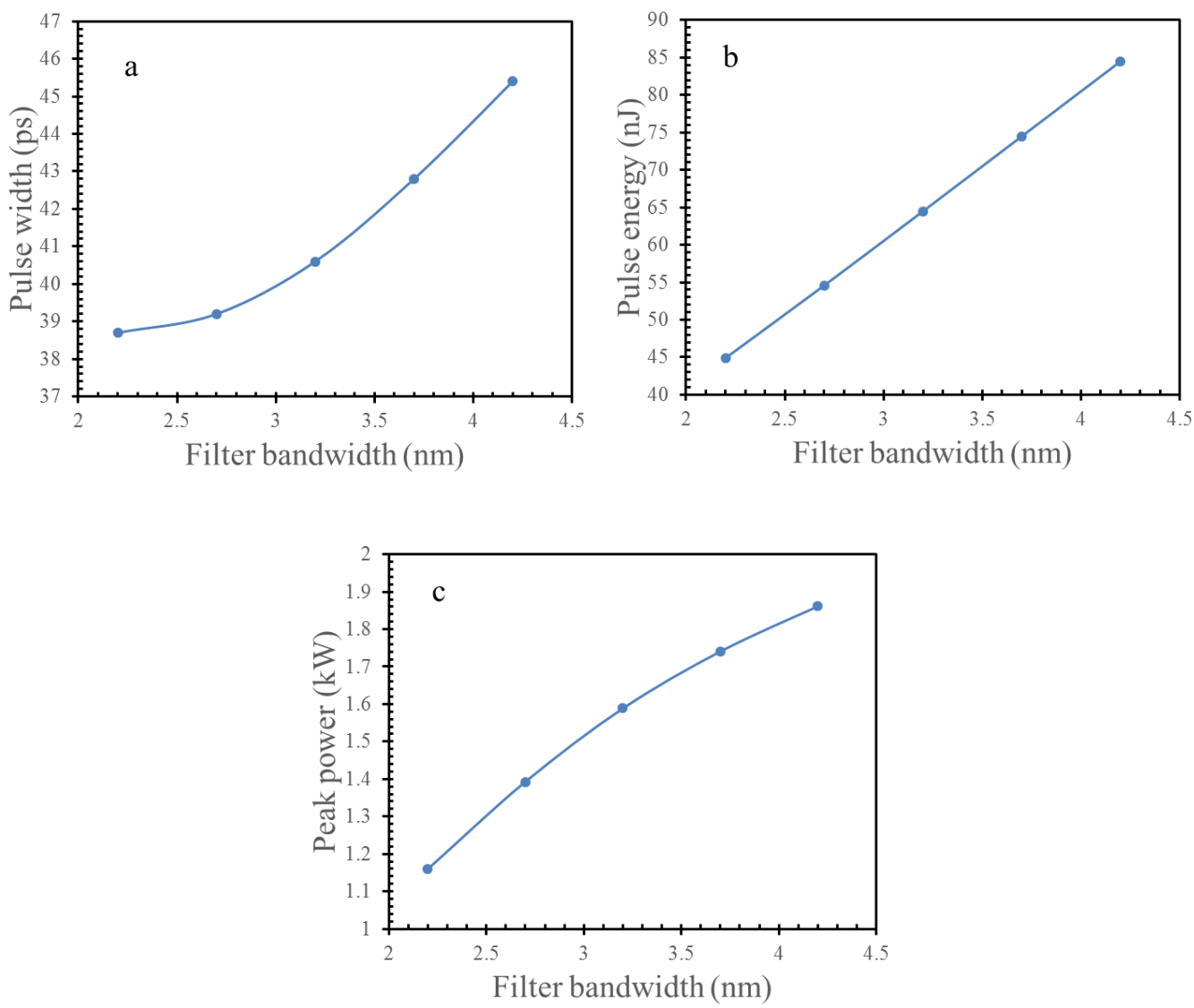

Fig. 3.8 Laser performance versus filter bandwidth: (a) pulse width, (b) pulse energy, (c) peak power.

\subsubsection{Main loop pump power}

The next laser parameter to be investigated is main loop pump power, which is proportional to the saturation energy $\left(E_{s}\right)$ of gain fiber. Figure 3.9 shows two simulation results with different $E_{\mathrm{s}}$, which are 0.7 and $0.3 \mathrm{~nJ}$. Other parameters are the same as described in Table 3.1\&3.2. Increasing the pump power will lead to a broadening of spectral width. However, the pump power cannot be increased without limitation. When the $\mathrm{E}_{\mathrm{s}}$ exceeds $0.7 \mathrm{~nJ}$, noise-like pulses were generated instead of the stable DS because of the ever-growing nonlinear effect. 
A spectral comparison between simulation and experiment is presented in Fig. 3.9(a). The experiment was carried out under the same cavity condition as the numerical simulation. The similar spectral width and SPM spectral ripple shape indicate a good agreement between the experiment and numerical simulation. The less symmetrical shape of the experimental spectrum could result from the improper length of the gain fiber. If the length of the gain fiber is too long, the short-wavelength component of the spectrum will be absorbed and converted into the long-wavelength component and thus lead to the asymmetrical shape of the spectrum.
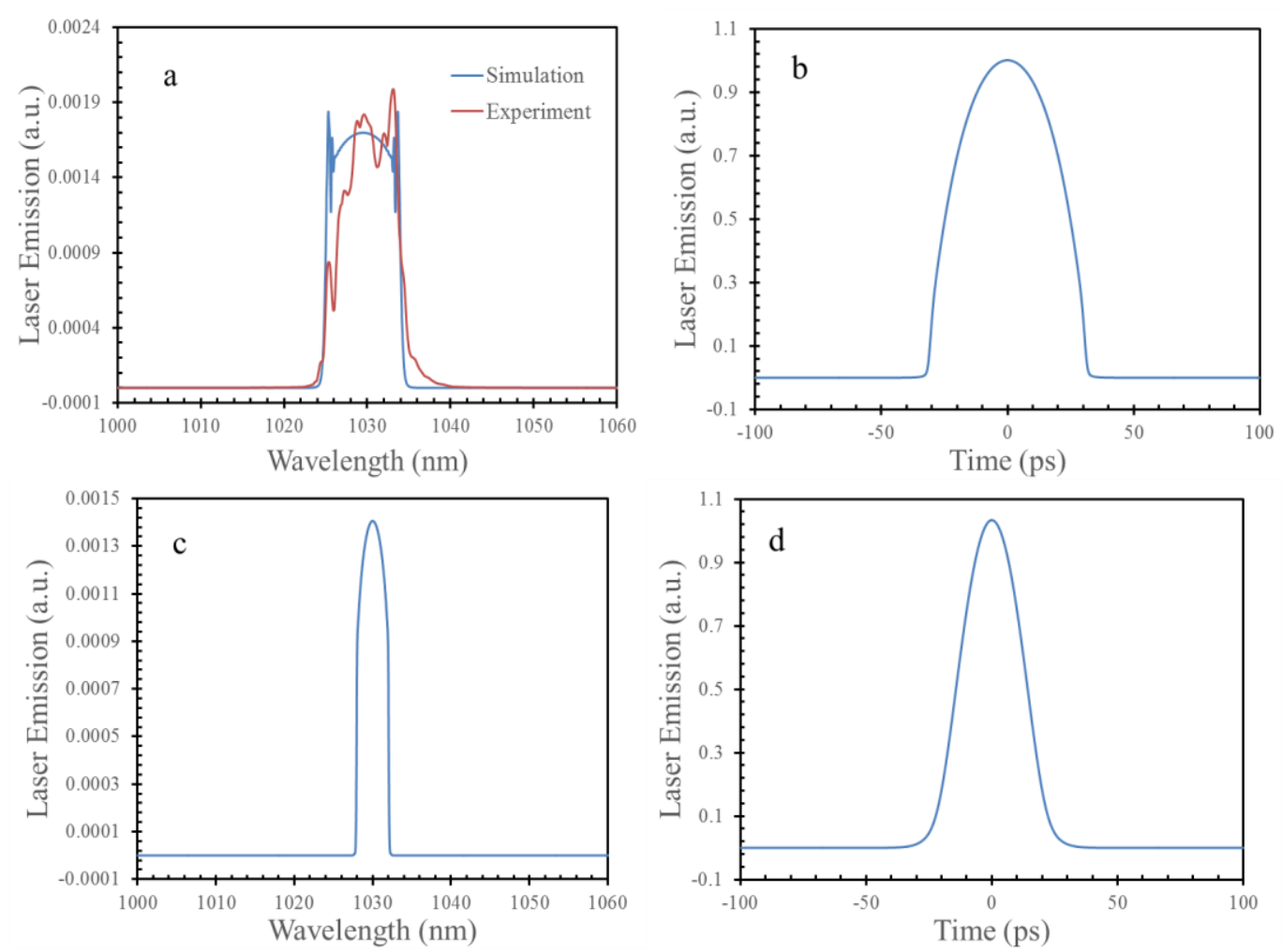

Fig. 3.9 Output spectrum and pulse shape with different saturation energy, with $0.7 \mathrm{~nJ}$ (a, b) and $0.3 \mathrm{~nJ}(\mathrm{c}, \mathrm{d})$; comparison between experiment and simulation (a).

The dependence of the laser output parameters on $\mathrm{E}_{\mathrm{s}}$ is summarized in Fig.3.10. The output ratio and extra PM 980 fiber length is fixed at $70 \%$ and $60 \mathrm{~m}$ for all the simulation in this section. The pulse width, pulse energy and peak power, all three parameters increase when the pump power rises [Fig 3.10] due to the 
increasing of SPM. Therefore, when all the other cavity parameter are fixed, one can increase the main loop pump power to get the higher output energy until the laser produces noise-like pulses.
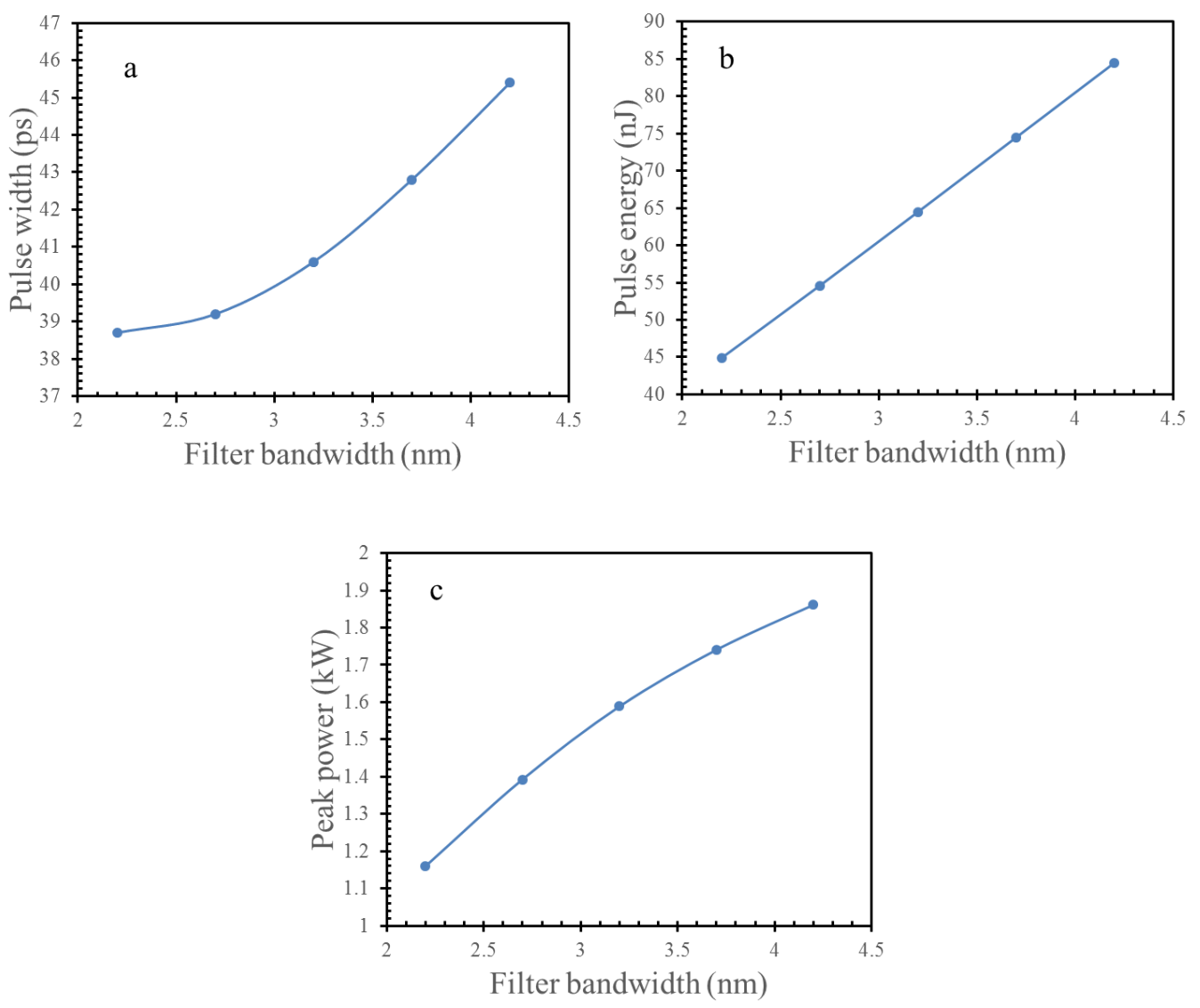

Fig. 3.10 Laser performance versus saturation energy: (a) pulse width, (b) pulse energy,

(c) peak power.

\subsubsection{NALM loop pump power}

The last laser parameter to be investigated is NALM loop pump power, which is proportional to the gain $(\mathrm{g})$ in Eq. 2.5. Figure 3.11 shows two simulation results with different $\mathrm{g}$, which are 3 and 5. Other parameters are the same as described in Table 3.1\&3.2. It can be seen that increasing the NALM pump power will lead to a pulse breaking phenomenon [Fig 3.11(d)]. The pulse splitting can be explained as following: when the NALM pump power increases, the spectral width and pulse width of the DS both increase due to the SPM. However, the spectral width 
broadening is limited by the gain bandwidth of Yb-ions. Once the spectral width reaches its maximum, its pulse width is also constrained and the corresponding DS pulse energy is clamped. Further increasing the pump, $\mathrm{P}_{\mathrm{NALM}}$, may not change the soliton pulse width but increase the gain for the dispersive waves which leads to the generation of a new soliton.
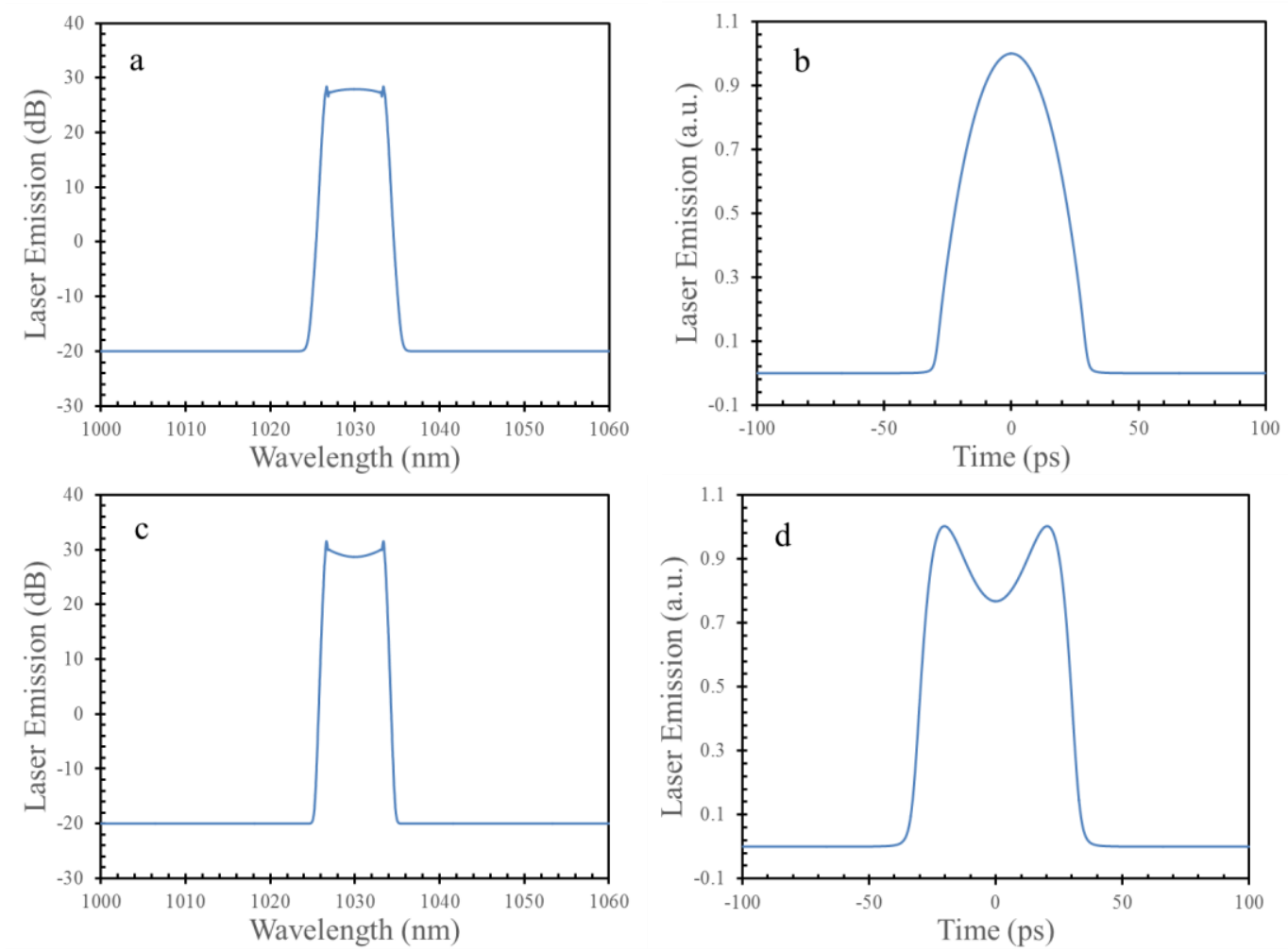

Fig. 3.11 Output spectrum and pulse shape with different NALM gain, with g $_{\text {NALM }}=3$ (a) and $\mathrm{g}_{\mathrm{NALM}}=5(\mathrm{~b})$

\subsubsection{Summary}

The simulations showed that longer cavity length, higher output ratio, higher pump power and broader filter bandwidth can lead to higher output pulse energy. However, all these parameters cannot be increased without limitation, otherwise noise-like pulses will be generated. Thus, a design guideline for the DS modelocked fiber lasers can be summarized: 
1. Determine the filter bandwidth and output ratio for the laser according to the simulations. In the case of NALM laser, filter bandwidth should be between 2-4 $\mathrm{nm}$ and output ratio could be $70 \%$ or $80 \%$.

2. Determine an approximate cavity length and build up the laser cavity.

3. Tune the pump power until you get a highest output energy as long as the laser is still at DS working regime.

4. Increase the cavity length by adding extra PM 980 fibers and then optimize the pump power.

5. Repeat step \#4 again until the working regime of DS become very tight by tuning the pump power. Then, this configuration gives you the highest output energy with the selected filter bandwidth and output ratio. 


\section{Chapter 4}

\section{SESAM mode-locked fiber laser}

In this chapter, I describe an all-fiber, environmentally-stable and ultra-compact ANDi fiber laser by using one mode-field-adaptor (MFA) to launch light onto the SESAM. A polarization-maintaining fiber loop mirror is used as a multifunctional component to realize the design of compact size. Self-starting and stable dissipative solitons mode-locking operation is achieved with $1.7 \mathrm{~nJ}$ pulse energy and 22 ps pulse width. A power stability test for over 4 hours confirms the environmentally-stable property of this laser.

\subsection{Introduction}

Among all the SAs or equivalent SAs, SESAM is most widely used in commercial mode-locked fiber lasers due to its relatively low-cost and welldeveloped production technology. However, it also has the drawbacks like relatively low damage threshold, uncertain long-time reliability and using bulk optics to focus the beam on its surface. Thus, environmentally-stable, compactsize and SESAM-based dissipative soliton fiber lasers are extremely demanded for the industrial application.

Several environmentally-stable SESAM-based DS fiber lasers have been reported recently. A self-starting self-similar all-PM components SESAM Yb-doped fiber laser was demonstrated in [74] with $1 \mathrm{~nJ}$ pulse energy; however dispersion management components (a pair of bulk gratings) complicate the laser configuration. Chong et al. reported an all-normal-dispersion (ANDi) SESAM mode locked fiber laser with $2.2 \mathrm{~nJ}$ pulse energy [75]. Although the laser is environmentally-stable, the bulk components undermine some of the benefits of fiber lasers. A high-power ANDi fs SESAM fiber laser was demonstrated by 
Lecaplain et al. using a LMA Yb-doped micro structure fiber [76]. Extreme high pulse energy up to $71 \mathrm{~nJ}$ is achieved, but the laser is not environmentally-stable and also contains lots of bulk components. The fiber lasers mentioned above may not be suitable as an ideal seed laser for industrial application.

In order to scale-up the pulse energy of SESAM-based DS fiber lasers, the beam spot launched onto the SESAM has to be enlarged to overcome the damage threshold of SESAMs. This can be accomplished by using a lens and a collimator. An environmentally-stable, compact-size and SESAM-based dissipative soliton fiber laser was demonstrated by me and Lei in 2014 [77]. However, the lens and collimator used to enlarge the beam size would influence the power stability, increase the laser cavity size and complicate the optical assembly. Such problems can be avoid by replacing the lens with a mode-field-adaptor (MFA).

An MFA is an essential device to efficiently transfer light from a standard single mode fiber to the $\mathrm{LP}_{01}$ mode of LMA fibers. Its performance is much superior to that of a regular splice, as an ordinary splice will produce significant loss and lead to a poor $\mathrm{M}_{2}$ factor. MFAs can generate lower losses than ordinary splices, thus ensuring higher power transmission compared to regular splicing. Therefore, using MFA to launch to light onto SESAMs can achieve the purpose of enlarging the beam size and maintain the all-fiber structure at the same time.

In this section, an all-fiber, environmentally-stable and ultra-compact ANDi fiber laser by using one MFA to launch light onto the SESAM was demonstrated. The MFA was used not only to achieve all-fiber structure, but also to enlarge the beam spot on the SESAM in order to obtain high pulse energy. A polarizationmaintaining fiber loop mirror is used as a multi-functional component to realize the design of compact size. Self-starting and stable dissipative soliton modelocking operation is achieved with $1.7 \mathrm{~nJ}$ pulse energy and 22 ps pulse width. The power stability test for over 4 hours confirms the environmentally-stable property of this laser. 


\subsection{Experimental setup}

The laser has a simple all-fiber configuration of only five components as illustrated in Fig. 4.1. The linear cavity is formed between a fiber loop mirror and a SESAM. The SESAM (from BATOP $\mathrm{GmbH}$ ) has a 33\% modulation depth, a $100 \mathrm{~nm}$ spectral bandwidth, and a relaxation time constant of $15 \mathrm{ps}$. An MFA is applied to enlarge the beam size on the SESAM for energy scale-up. One side of the MFA is a HI 1060 fiber, which is spliced to the rest PM cavity; the other side is a 20/400 LMA fiber, which has a flat cleave on the end and butt-connected to the SESAM directly. The gain medium of the laser is a piece of highly Yb-doped PM single mode fiber, $20 \mathrm{~cm}$ in length, with peak absorption of $170 \mathrm{~dB} / \mathrm{m}$ at 976 $\mathrm{nm}$. The pump source is a $400 \mathrm{~mW}$ single mode fiber pigtailed diode laser emitting at $976 \mathrm{~nm}$, which is coupled into the laser cavity through a 976/1030 PM multiplexer (WDM) (both axes pass). Rest of the fibers are PM980 fiber and the whole round-trip cavity length is $15.4 \mathrm{~m}$, corresponding to an overall cavity dispersion of $0.37 \mathrm{ps}^{2}$ at $1033 \mathrm{~nm}$.

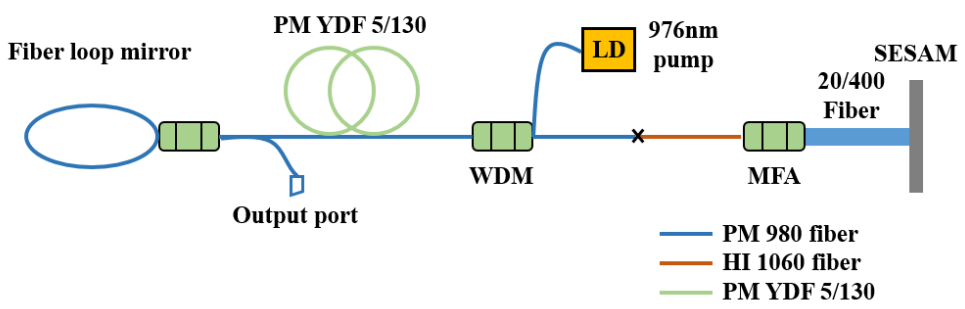

Fig. 4.1 Schematic diagram of the SESAM mode-locked fiber laser.

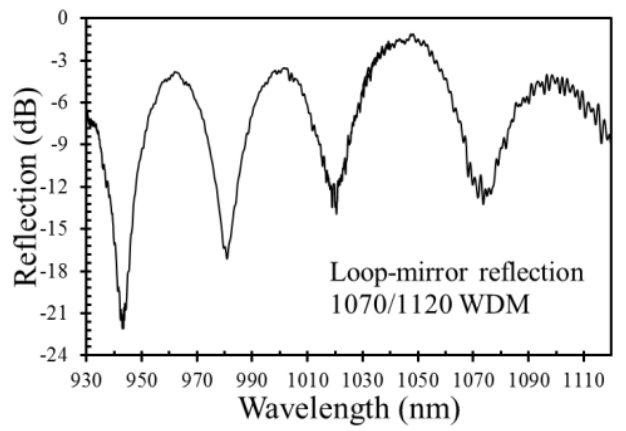

Fig. 4.2 Reflectivity spectrum of the PM loop mirror. 
A $2 \times 2$ PM fiber 1070/1120 nm WDM is employed to build a fiber loop mirror [75]. Two ports from one end are spliced together to form a loop, while the one port from the other ends is spliced to the cavity, and the other port is used as an output. Fig. 4.2 shows the slow axis reflectivity spectrum of the fiber loop mirror measured with an ASE source from $920 \mathrm{~nm}$ to $1120 \mathrm{~nm}$. A periodical reflectivity variation could be observed. The peak wavelength locates at $1044 \mathrm{~nm}$ with peak transmission (coupling efficiency) of about $73 \%$ and $30 \mathrm{~nm} 3 \mathrm{~dB}$ bandwidth between $1032 \mathrm{~nm}$ to $1060 \mathrm{~nm}$, which could provide strong spectral filtering of the highly chirped pulse to stabilize the mode locking operation. Thus, the fiber loop mirror serves a multiple purposes: as a highly reflective cavity mirror, a spectral bandpass filter and an output coupler.

\subsection{Experimental results and analysis}

Self-starting stable mode locked operation was obtained when the pump power was adjust between 59 to $222 \mathrm{~mW}$. The output power increases almost linearly with the pump power, as shown in Fig. 4.3(a), which gives a slope efficiency of $12 \%$. The maximum average output power of $21.7 \mathrm{~mW}$ was obtained at a pump power of $222 \mathrm{~mW}$. Further increase of the pump power led to a noise-like soliton. The pulse repetition rate was measured to be at $13 \mathrm{MHz}$ which gave the pulse energy of $1.7 \mathrm{~nJ}$.

The spectra of the mode-locked laser output at three different output power are shown in Fig. 4.3(b). Steep rising and falling edges could be observed in the spectrum under all three conditions, which is attributed to the dissipative solitons. Broader $10 \mathrm{~dB}$ bandwidth can be observed with higher output power. The central wavelength is at $1033 \mathrm{~nm}$ with a $10 \mathrm{~dB}$ bandwidth of $4.1 \mathrm{~nm}, 4.8 \mathrm{~nm}$ and $5.2 \mathrm{~nm}$, corresponding to $2.3 \mathrm{~mW}, 10.0 \mathrm{~mW}$ and $21.7 \mathrm{~mW}$ output power respectively. The spectrum broadening is due to the effect of self-phase modulation (SPM). Higher output peak power will lead to higher SPM, which will add more new frequency content into the spectrum. In order to compare the pulse peak power under different pump power, the pulse widths also need to be measured, which will be 
presented later. As the pump power increased, some sidebands appeared on the both side of the spectrum, while the central part of the spectrum remained the same. As the pump power increased further, the sidebands became more prominent, however it was still $30 \mathrm{~dB}$ less than the main spectrum peak at the highest output power, which did not influence the performance of the modelocked laser.
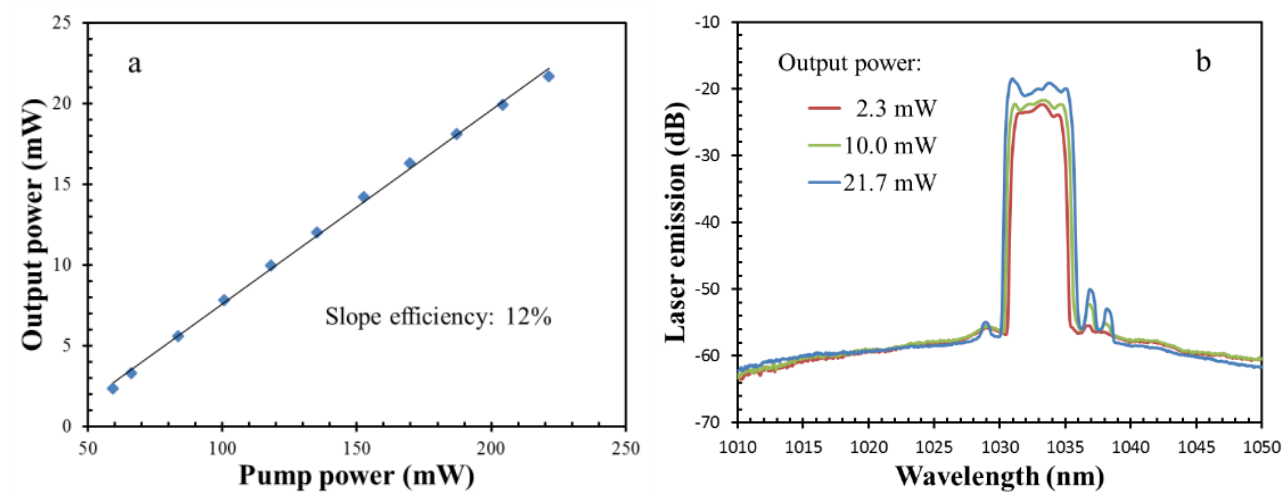

Fig. 4.3 (a) Laser output power as a function of pump power and (b) Output spectra of the mode locked laser under three different output powers.

The autocorrelation trace of the chirped pulses with two different output power was measured with an auto-correlator (Femtochrome FR 103-XL) as shown in Fig. 4.4. The measured pulse widths were $13.6 \mathrm{ps}$ and $22 \mathrm{ps}$ for output power of $2.3 \mathrm{~mW}$ and $21.7 \mathrm{~mW}$, respectively. Thus, the corresponding pulse peak powers were $13 \mathrm{~W}$ and $77 \mathrm{~W}$. The increasing of pulse peak power is due to the different increasing ratio between pulse energy and pulse width. The energy increased 9.4 times while the pulse width only increased 1.6 times, when the pump power increased from $59.6 \mathrm{~mW}$ to $221.5 \mathrm{~mW}$. Therefore, higher peak power under higher pump power will induce severer SPM, which will result in the spectrum broadening as shown in Fig. 4.3(b). Both measured pulse shapes match well with Sech fuctions. The pulse train measured with a photodetector (1 GHz bandwidth) is also shown in Fig. 4.3(b). It exhibits a pulse spacing of 76.9 ns, which matches well with the pulse round trip time of a $15.4 \mathrm{~m}$ long cavity length. 

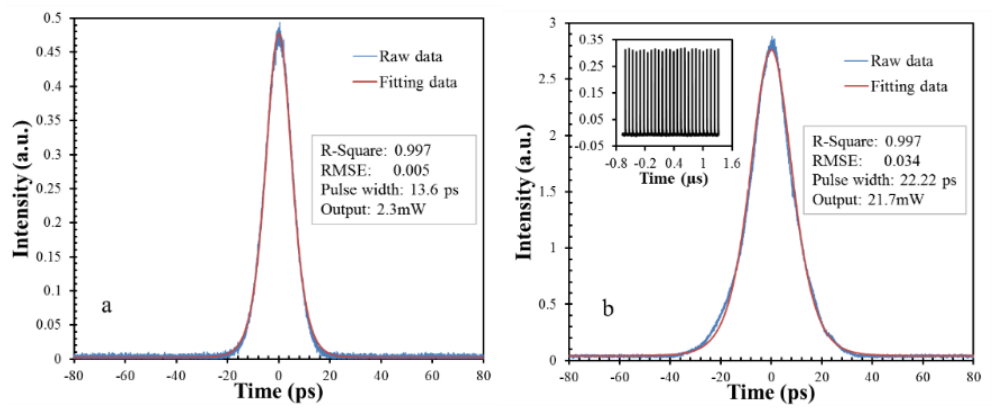

Fig. 4.4 Auto-correlator trace with an output power of (a) $2.3 \mathrm{~mW}$ and (b) $21.7 \mathrm{~mW}$.

The radio-frequency (RF) spectrum around the fundamental repetition is shown in Fig. 4.5(a), measured with a high resolution RF-spectrum analyzer. No obvious residual sidebands caused by Q-switched mode-locking could be observed. A 70 $\mathrm{dB}$ signal-to-noise ratio indicated excellent mode-locking stability and low pulse timing jitter. The RF spectrum consisting of a comb of harmonics, which corresponds to the repetition frequency of $12.96 \mathrm{MHz}$, is shown in Fig. 4.5(b). The corresponding resolutions for the RF spectrum around the fundamental and harmonics repetition were $10 \mathrm{~Hz}$ and $1 \mathrm{KHz}$, respectively.
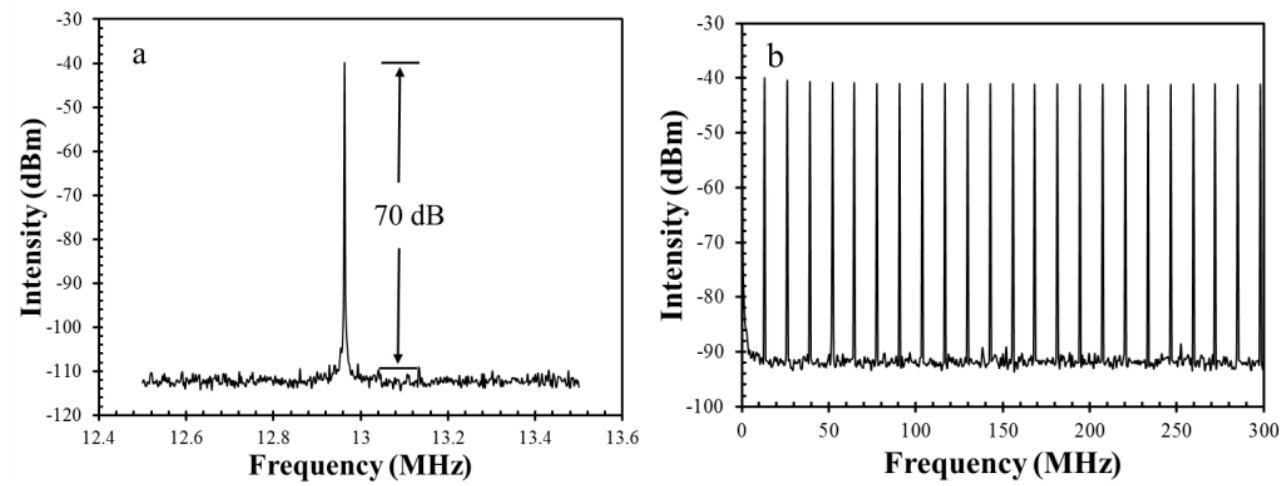

Fig. 4.5 Radio-frequency (RF) spectrum (a) around the fundamental and (b) harmonic repetition rates of the mode locked laser.

The power stability test of the mode-locked laser for over $4 \mathrm{~h}$ is shown in Fig. 4.6. A mean output power of $\sim 21.7 \mathrm{~mW}$ with $\sim 0.22 \mathrm{~mW} \mathrm{rms}$ instabilities over $4 \mathrm{~h}$ represents a $1 \%$ relative rms noise with respect to the average signal power, indicating the good power and mode-locking stability of the laser. For higher power operations, the beam spot size on the SESAM could be further increased to 
increase the damage threshold, for example, replacing the MFA with output fiber of $25 / 250$ size. Benefiting from the all-fiber and PM configuration, the mode locking operation could be maintained by tapping the fiber and by changing the environmental temperature.

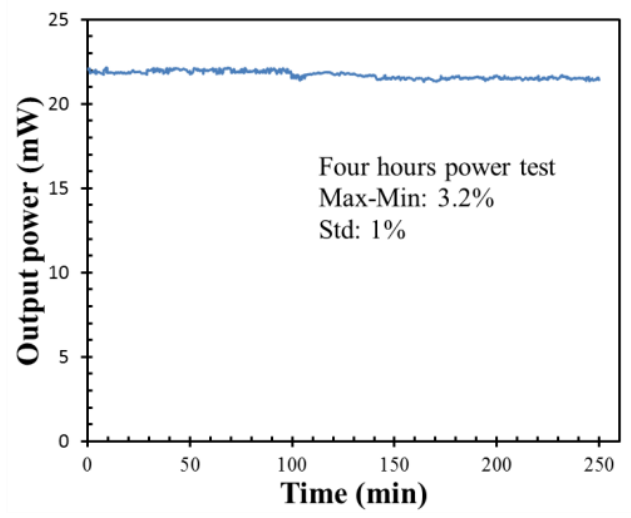

Fig. 4.6 Output power stability tested with a duration of $4 \mathrm{~h}$.

\subsection{Summary}

In summary, an all-fiber, environmentally-stable, ultra-compact and SESAMbased linear cavity mode-locking fiber laser has been demonstrated. A mode-field adaptor is employed to enlarge the beam size and launch light onto the SESAM in order to increase the output pulse energy. A polarization-maintaining fiber loop mirror is used as a multi-functional component (as a highly reflective cavity mirror, a spectral band-pass filter and an output coupler) to realize the design of compact size. Self-starting and stable dissipative soliton mode-locking operation is achieved with $1.7 \mathrm{~nJ}$ pulse energy and $22 \mathrm{ps}$ pulse width. Power stability test over four hours shows the laser's excellent environmental stability, which proves it to be an ideal seed laser for industrial application. 


\section{Chapter 5}

\section{NALM mode-locked fiber laser}

In this chapter, I demonstrate that stable DS mode-locking can be achieved in a long cavity ring laser configuration with an NALM. $32 \mathrm{~nJ}, 615$ fs de-chirped

pulse width were obtained with the Raman signal suppressed below -20 dB. With the increase of pump power, a transition of mode-locking from noise-like pulses with a strong Raman scattering to stable DS mode-locking accompanied by an abrupt $16 \mathrm{~dB}$ reduction of Raman emission was observed. The DS pulse can be de-chirped to 615 fs. This mode-locking is self-starting and the mode-locked pulse train shows excellent stability.

\section{$5.1 \quad$ Introduction}

Recently, DS mode-locked fiber lasers in all-normal dispersion cavities have been extensively studied owing to their high pulse energy and linearly chirped pulse, which can be further compressed. Various mode-locking mechanisms have been studied to achieve stable DS operation. Among them, NPR offers high pulse energy and SESAM offers environmentally-stable mode-locking [13, 80]. However NPR mode-locking often requires free-space components or polarization controllers which may not be suitable in industrial settings. The SESAM modelocked laser suffers from the low energy damage threshold and limited lifetime. Recently, Broderick et al. reported an all-fiber mode-locked laser with an NALM which achieved environmental-stable output with pulse energy of $16 \mathrm{~nJ}$ [31, 35, 36], much higher than those of SESAM mode-locked lasers. Their studies suggested that increasing the cavity length could be an effective method for scaling-up pulse energy and avoiding the wave-breaking as the NALM would amplify the stretched pulses. On the other side, it is well-known that long fiber length will enhance the non-linear effects such as stimulated Raman scattering 
(SRS). It is apparent that the interaction between the high energy DS pulse and non-linear effects becomes an interesting topic in the further scaling up of DS pulse energy.

By extending the cavity length, Raman-driven destabilization of mode-locked lasers was reported recently [73], - the laser operated in a noise-like regime in the presence of strong Stokes signal. The authors of ref. [73] concluded that SRS could constitute a fundamental limit to the energy scalability of the long cavity mode-locked fiber laser. However, another study, reported by Kharenko et al., showed that highly chirped stable DS can be obtained even with the presence of strong Raman scattering [37]. In their cavity design, a combination of a short piece of single-mode fiber and a long piece of polarization maintaining (PM) single-mode fiber was used to lengthen the cavity while prevented excessive NPR from breaking the wave. The pulse energy of $23 \mathrm{~nJ}$ was obtained, in which, $17 \mathrm{~nJ}$ belongs to DS. In their subsequent theoretical and experimental studies, they showed that the DS and Raman pulse form a complex, - the DS amplified the Raman pulse and the Raman pulse stabilized the DS via temporal and spectral filtering [38]. It should be noted that the laser cavity design used in ref. [37, 38] was different from that of ref. [36]; the former had no narrow-band spectral filtering at $1030 \mathrm{~nm}$ so the Raman signal re-entered the cavity after each round trip and the latter had a $1.7 \mathrm{~nm}$ band-pass filter at DS wavelength so the Raman signal was filtered out in each round trip. From practical point of view, the laser design in ref. $[37,38]$ employed a polarization controller for mode-locking while the lasers used in ref. [31, 35, 36, 73] were self-starting. Despite the success of Kharenko et al. in achieving stable DS mode-locking in the presence of strong Raman signal, the further energy scale-up was still limited by the SRS, not due to the stability of DS, but due to the energy diverging from DS to Raman signal. These studies clearly indicated the importance of interaction between SRS and DS in a long cavity laser - one needs to find a way to suppress the Raman signal while sustaining stable DS mode- locking at a high intra-cavity power level. 


\subsection{Experimental setup}

The design of the laser, depicted in Fig. 5.1, uses PM fiber and all PM components. The main loop consists of a 980/1040 nm wavelength division multiplexer (WDM \#1), a $50 \mathrm{~cm}$-long high-concentration Yb-doped PM fiber with an estimated core absorption of $750 \mathrm{~dB} / \mathrm{m}$ at $976 \mathrm{~nm}$, a segment of standard PM single-mode fiber (60 m), a PM isolator, a 30/70\% PM output coupler with the $70 \%$ transmission port used as an output and a narrow bandpass filter centered at $1030 \mathrm{~nm}$ with a bandwidth of $2.76 \mathrm{~nm}$. The NALM loop is connected to the main loop by a 40/60 PM coupler between the isolator and the output coupler. The loop consists of a $30 \mathrm{~cm}$-long high-concentration Yb-doped PM fiber, a 980/1040 nm WDM (\#2), and a segment of PM980 single mode fiber (2 m). The overall cavity length is $81 \mathrm{~m}$.

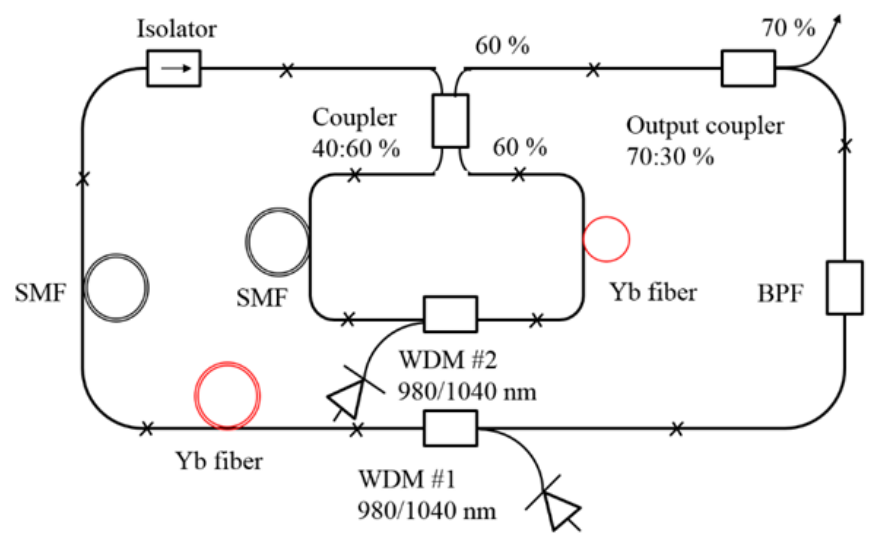

Fig. 5.1 Scheme of an all-fiber all-PM NALM DS fiber laser.

The autocorrelation trace of the chirped pulses was measured with an autocorrelator with a limited detecting range of 80 ps (Femtochrome FR 103-XL). The pulse train was measured with a photodetector ( $1 \mathrm{GHz}$ bandwidth). The radio-frequency $(\mathrm{RF})$ spectrum was measured with a high resolution RF-spectrum analyzer (Agilent N9320B). A grating pair with 1200 lines/mm (Newport) is used to externally compress the chirped pulses into fs pulses. 


\subsection{Experimental results}

In order to understand the interaction between the DS and Raman signals in a long-cavity ring laser, the emission spectra at the different pump power of the main loop, the mode-locked pulse train and its RF spectra before pulse compression were measured. The auto-correlator traces were recorded before and after pulse compression. Fig. 5.2 shows the selected spectra of the laser emission at four different main loop pump power, $\mathrm{P}_{\text {main, }}$, while the pump power in the NALM loop, $\mathrm{P}_{\mathrm{NALM}}$, is fixed. The laser starts to produce noise-like pulses with a characteristic smooth triangle-shaped spectrum peaked at $1030 \mathrm{~nm}$ and with a bandwidth of $2.0 \mathrm{~nm}$, as shown in Fig. 5.2(a), when $P_{\text {main }}$ reaches $162 \mathrm{~mW}$. The Raman signal at $1078 \mathrm{~nm}$ is visible, nevertheless at more than $40 \mathrm{~dB}$ below the laser emission. As the $\mathrm{P}_{\text {main }}$ increases, the Raman peak at $1078 \mathrm{~nm}$ increases rapidly. When $\mathrm{P}_{\text {main }}$ reaches $288 \mathrm{~mW}$, the Raman peak grows to $\sim 30 \mathrm{~dB}$ above the noise level with a bandwidth of $6.6 \mathrm{~nm}$, as shown in Fig. 5.2(b). The pulses remain in the noise-like regime. However, a transition of the mode-locking state is observed with the further increase of $\mathrm{P}_{\text {main }}$ to $320 \mathrm{~mW}$. The mode-locking changes from noise-like into DS whose spectrum in Fig. 5.2(c) shows the steeper edges with characteristic ripples on both side of the spectrum. The bandwidth of the DS is broadened significantly to $8.0 \mathrm{~nm}$ at $-3 \mathrm{~dB}$. This transition is accompanied by a $16 \mathrm{~dB}$ drop of the Raman peak. The mode-locking would remain in the DS regime with the further increasing of the pump power. Fig. 5.2(d) shows the broadened DS emission spectrum and the growing Raman signal when the pump power, $\mathrm{P}_{\text {main, }}$, reaches its maximum output of $445 \mathrm{~mW}$. The average power of the laser reaches $79 \mathrm{~mW}$ that gives the pulse energy of $32 \mathrm{~nJ}$. Notice that the Raman emission is $20 \mathrm{~dB}$ below the DS emission. 

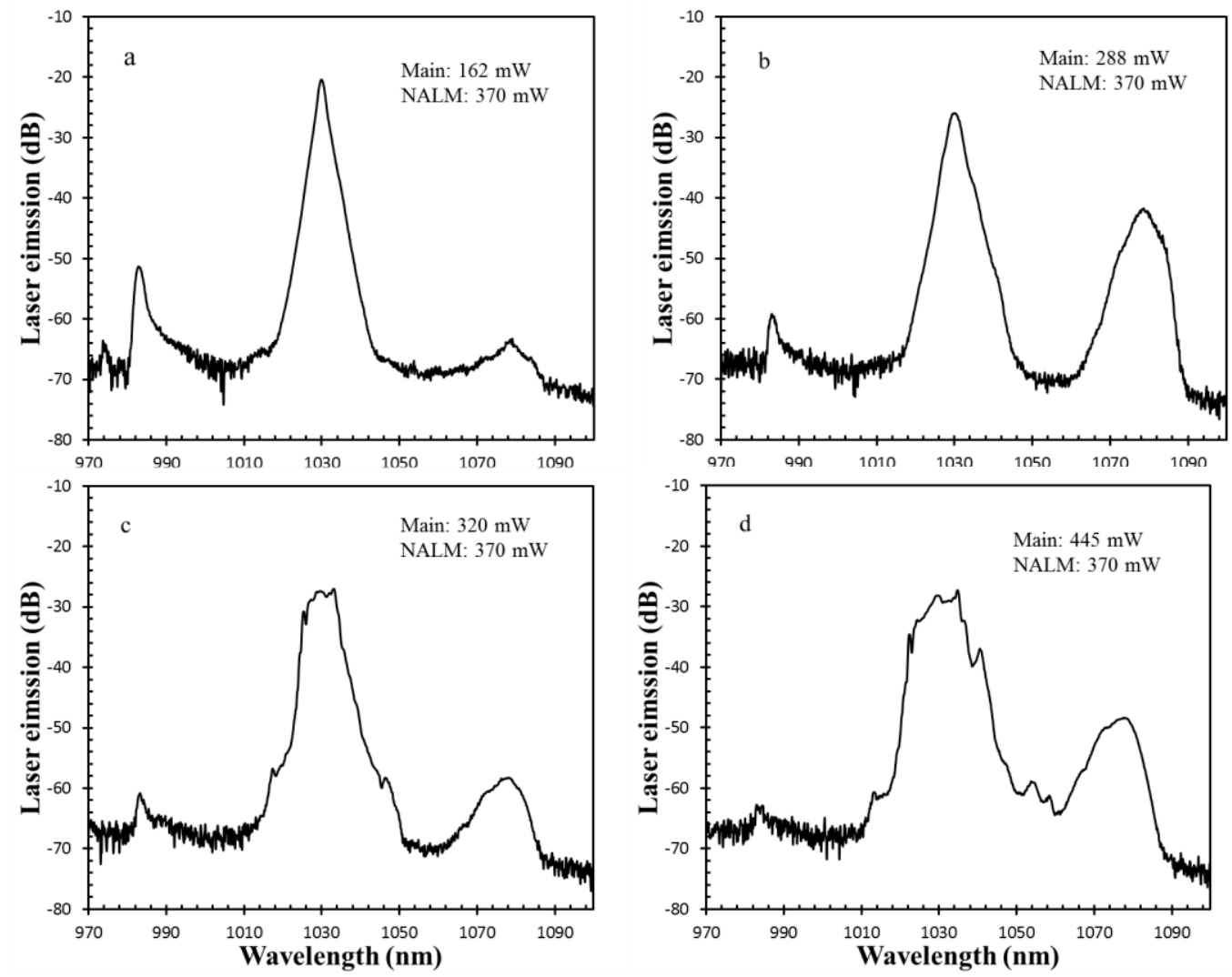

Fig. 5.2 Spectrum evolution of the NALM mode-locked laser.

The mode-locked pulse train is measured and shown in Fig. 5.3. The measured pulse spacing of $405 \mathrm{~ns}$ corresponds to a repetition rate of $2.47 \mathrm{MHz}$, matching well with the cavity length. No pulse splitting was observed. The RF spectra for both noise-like pulses and DS solitons were measured at a resolution of $10 \mathrm{~Hz}$ for the fundamental frequency, as shown in Fig. 4. For the noise-like pulses, its fundamental frequency spectrum in Fig. 5.4 (a) shows two side peaks as the result from the amplitude modulation. For the DS soliton, not only the signal-to-thenoise ratio is $10 \mathrm{~dB}$ higher than that of noise-like pulses, the side peaks also disappears completely. The RF spectrum with a high signal-to-noise ratio of 80 $\mathrm{dB}$ indicates high pulse-to-pulse stability of the DS soliton. 


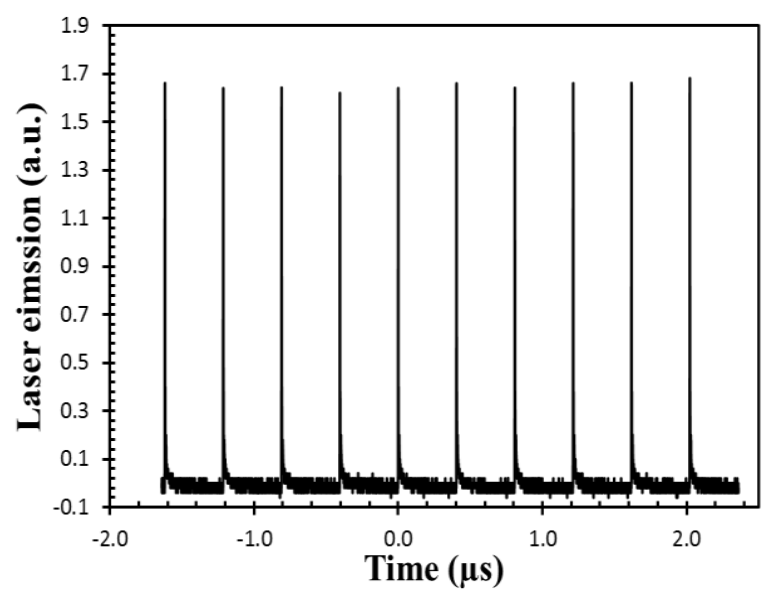

Fig. 5.3 Output pulse train of the NALM mode-locked laser in DS regime.
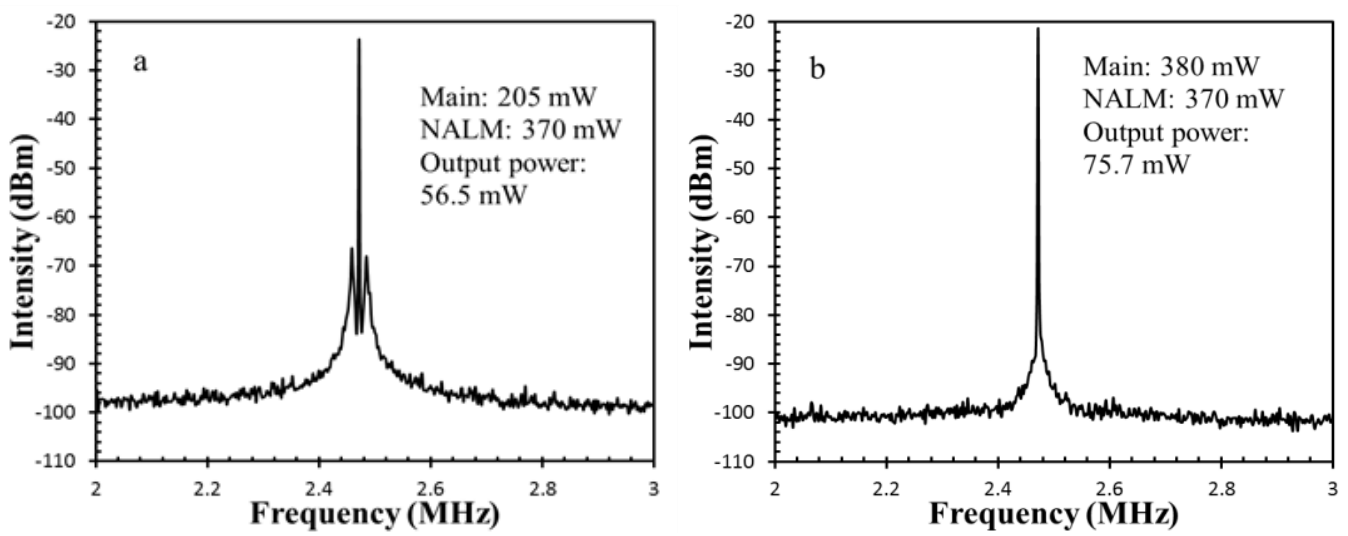

Fig. 5.4 RF spectrum at the fundamental repetition rate in the noise-like regime (a), and in the dissipative soliton regime (b).

In order to have a better understanding of the observed transition, the areas of the DS and Raman spectral peaks were integrated and plotted in Fig. 5.5. In the noiselike regime, the power of the Raman signal increases from negligible to $2.4 \mathrm{~mW}$ while the power of noise-like pulses decreased from $66.5 \mathrm{~mW}$ to $53 \mathrm{~mW}$ when the pump power $\mathrm{P}_{\text {main }}$ increases from $210 \mathrm{~mW}$ to $300 \mathrm{~mW}$. This confirms what reported in ref. [73] that the Raman signal became the limiting factor for energy scalability. However, when $\mathrm{P}_{\text {main }}$ increased to $320 \mathrm{~mW}$, the noise-like pulses switched to the DS regime with power surging from $53 \mathrm{~mW}$ to $73.6 \mathrm{~mW}$. The power of Raman emission dropped from $2.4 \mathrm{~mW}$ to $0.07 \mathrm{~mW}$. This switching from noise-like to DS soliton is interesting, not only for the understanding of 
mode-locking mechanism but also for the power scaling up of DS. The transition could be attributed to the shift of the transfer function of the NALM loop which is the mode-locking element in this laser. With the increase of pump, the power in the NALM loop also increases which shifts the maximum transmission to favor DS mode-locking. The transmitted power of NALM has been described in Eq. 2.5. Fig. 5.6 shows the transmitted peak power of the NALM at three different gains $(g=15,20,25)$ as a function of input peak power. The coupler ratio $\alpha=0.4$, loop length $\mathrm{L}=4 \mathrm{~m}$, and the wavelength is $1.03 \mu \mathrm{m}$. All three curves show a sinusoidal transmission profile with increasing modulation amplitudes. Higher gain leads to higher transmitted peak power and more transmitted peaks in a given input power range. If the input peak power is at the rising edge of the transfer function, the peak of the pulse will have the higher transmitted power than the rest of the pulse. Thus the pulse width of the output soliton will be trimmed, i.e. the NALM serves as a saturated absorber. In contrary, if the peak power of the incident soliton is at the falling edge, the peak of the pulse will have lower transmission than the rest the pulse. The pulse width will be broadened instead.

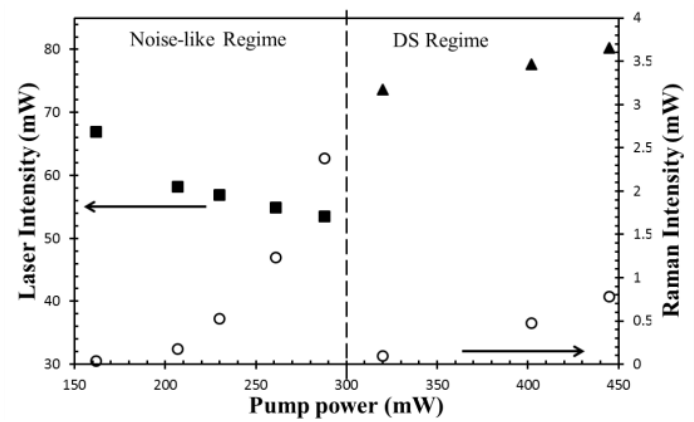

Fig. 5.5 Integrated power of the noise-like pulses ( $\boldsymbol{\square})$, DS ( $\mathbf{\Delta})$ and Raman emissions ( O) versus main loop pump power.

The transition from noise-like to DS by can be explained Fig. 5.6. At low input peak power, the NALM modulation is not sufficient to cut out the two pulse wings so the laser produces noise-like pulses [78]. At high input peak power, the higher modulation depth provided sufficient saturable absorption function for the laser to switch to DS operation. 


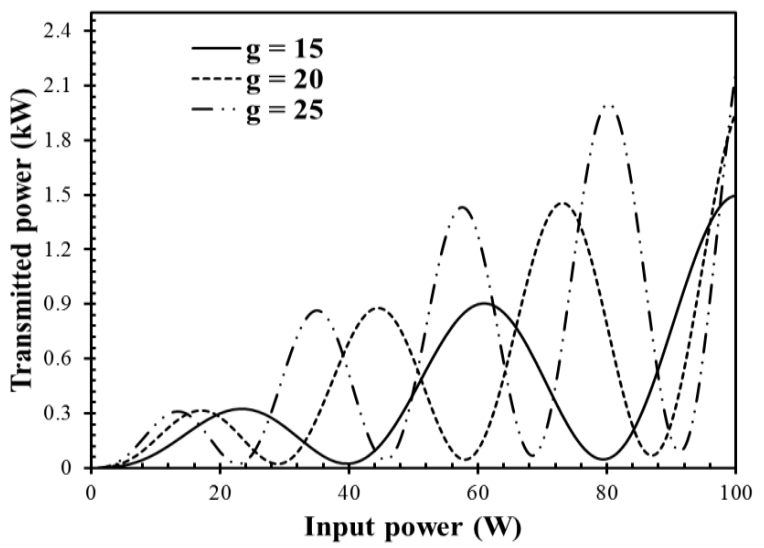

Fig. 5.6 Simulation of NALM's transmitted power against input power.

The reduction of the Raman signal accompanied with this transition may be explained by the reduction of the spectral power density of DS as its FWHM increased from $3.34 \mathrm{~nm}$ to $8.0 \mathrm{~nm}$ after transition. In addition, the peak power difference in two regimes may also contribute to the Raman signal reduction. The noise-like pulse contains many irregular short pulses whose peak power is much higher and thus is capable of inducing high Raman signal. When the modelocking transits to DS regime, its broad pulse width of $~ 60$ ps lowers the peak power that results in the lower induced Raman signal. In Fig. 5.2(d), the integrated Raman signal counts about $1 \%$ of power in total laser emission. As the pump power increases further in DS regime, the Raman signal begins to increase again, nevertheless at a slower rate than in the noise-like regime as shown in Fig. 5.5, which indicates that further DS energy scaling may still be limited by SRS.

The autocorrelation trace is presented in Fig. 5.7. For the noise-like pulse shown in Fig. 5.7(a), which shows a narrow sharp peak in the center of a broad pulse. Such feature is the characteristics for the noise-like pulses. In contrast, such a narrow peak is absent in the autocorrelation trace of DS pulses shown in Fig. 5.7(b). The pulse was cut on both sides due to the limited detection range of our autocorrelator ( $\sim 80 \mathrm{ps})$. A pair of volume grating was used to compress the DS pulses to fs. The fully de-chirped pulse is shown in Fig. 5.7(c) with a DS pulse width of $615 \mathrm{fs}$, which proves the linearly-chirp characteristics of the DS. 

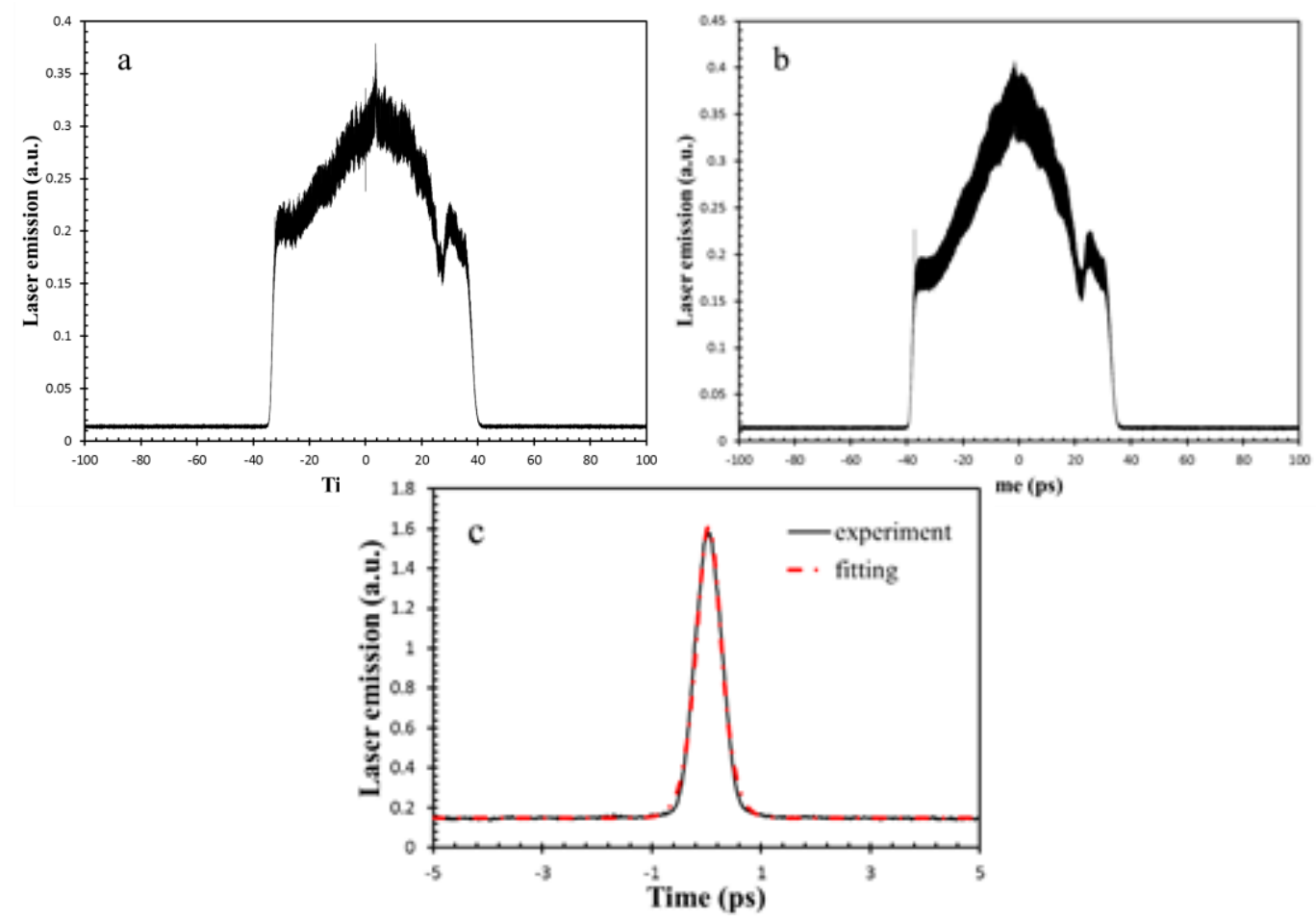

Fig. 5.7 Autocorrelation trace of the mode-locked laser (a) Noise-like; (b) DS; and (c) fully dechirped DS.

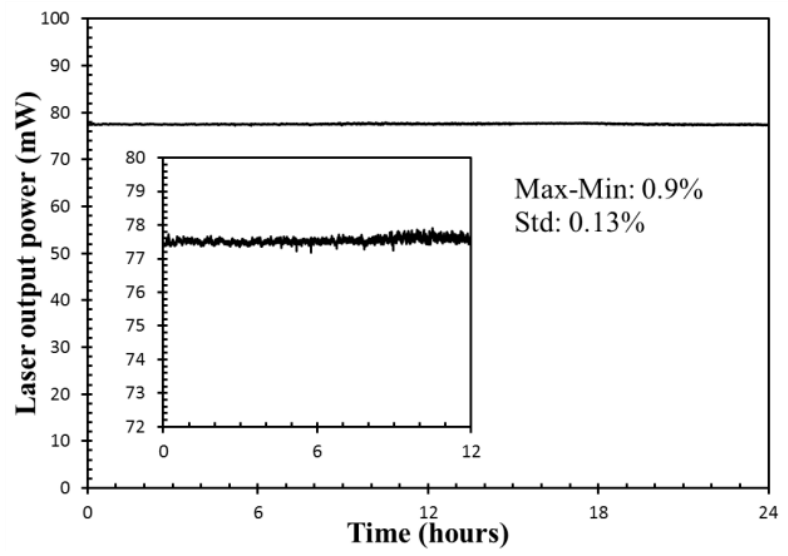

Fig. 5.8 Output power stability test in a duration of 24 hours.

To demonstrate the power stability of the DS in the presence of the Raman emission, the output power was measured over 24 hours as shown in Fig. 8. An average output power of $77.6 \mathrm{~mW}$ was measured which shows only $\sim 0.1 \mathrm{~mW}$ rms power variation, about $0.13 \%$ relative RMS noise. This test, together with 
the RF spectrum of DS presented in Fig. 5.4(b), demonstrated the excellent stability of the DS emission despite the presence of Raman emission.

\subsection{Summary}

I described a transition in mode-locking from unstable noise-like pulses to stable high energy DS in the presence of Raman signal in a long cavity NALM modelocked fiber laser. The DS of $32 \mathrm{~nJ}$ with a de-chirped pulse width of 615 fs was generated when the pump power surpassed the value that generated the noise-like pulses. The DS energy exhibited a leap after the transition compared with that of the noise-like pulses and the onset of DS was accompanied with an abrupt $16 \mathrm{~dB}$ reduction of Raman signal.

These results clearly showed that pulse energy scale-up can be realized by the extension of cavity length as long as the pump power exceeded the level that emitted noise-like pulses. The $32 \mathrm{~nJ}$ DS generated is a factor of 2 higher than the pulse energy of $16 \mathrm{~nJ}$ reported in [35] and 60\% higher than what was reported in [73]. Even in the presence of Stokes signal, the DS showed remarkable stability over a relatively long time. Our results further show that, in addition to the interactions between gain, nonlinearity and dispersion in the main loop, the transfer function of NALM at different power levels is also critical to the state of mode-locking. The understanding of these interactions will lead to better control over the mode-locking process and therefore achieving higher pulse energy. Due to its all-fiber configuration, linearly-chirped pulse and high pulse energy, this laser can be used as a stand-alone oscillator for scientific applications, or used as a seed laser for further amplification for industrial applications. 


\section{Chapter 6}

\section{Generation of multiple dissipative solitons}

In this chapter, a unique multi-pulsing phenomenon is investigated in the NALM dissipative soliton mode-locked fiber laser. $56.8 \mathrm{~nJ}$ single DS pulse operation can be achieved under certain pump power. Further increasing the pump power will lead to stable multi-pulsing. The separating distance between the split pulses can be step-tuned by changing the NALM loop's pump power.

\subsection{Introduction}

Besides being good pulsed oscillators, DS mode-locked fiber laser also provides an ideal platform for the studies of nonlinear dynamics because of the interaction between different physical phenomena inside the fiber cavity. Unlike traditional solitons which can be formed by the balance of nonlinear and anomalous dispersion inside the laser cavity [18], DS is based on a composite balance between several factors, such as chromatic dispersion, Kerr nonlinearity, bandwidth-limited gain from doped gain fibers, and intensity-dependent loss from saturated absorbers and spectral filtering [79]. The interplay among these physical effects would not only lead to stable single pulse dissipative solitons, but also produces some multi-pulsing phenomenon, such as soliton molecules, soliton rains and harmonic mode-locking [79].

A distinct characteristic of DS is their ability to aggregate into stable bound states, called "soliton molecules" [80]. In soliton molecules, several identical soliton pulses with similar intensity tend to group themselves into a stable packet. The time separation between two pulses is in the range of ps, which is much smaller than the cavity round-trip time (usually in the tenth of ns). Since the total energy of a soliton molecule is slightly different from the sum of the energies of its 
soliton constituents, dissipative processes are involved in progress of forming a soliton molecule, which implies the existence of a binding energy. The forming of soliton molecules is explained by self-phase locking of these pulses because the interaction of optical pulses through their tails is phase-sensitive [81, 82]. Other soliton molecule related dynamics such as long-period soliton pulsations [83], soliton collisions [84], and vibrations of soliton pairs [85] have also been explained by dissipative soliton concepts and models. Soliton rains is also one multi-pulsing phenomenon, generated in a regime when soliton pulses and $\mathrm{CW}$ components are of comparable strength [86]. These two components not only coexist but also interact in a dramatic way. In soliton rains, new dissipative soliton pulses will keep being generated from noise background with much lower intensity compared to the soliton bound. These pulses will drift at almost constant velocity until they reach a condensed soliton phase [87]. Harmonic mode-locking can also be considered as a multi-pulsing phenomenon, in which, one pulse splits into two equal-spacing pulses, which will lead to a multiple times of repetition rate. Such equal-spacing pulse-break can be understood as resulting from a weak repulsive force attributed to the gain medium depletion and the recovery time between subsequent solitons [88]. Another observation of a special multi-pulsing was reported by Zhao et al. in a gain-guided DS fiber laser, in which multipulsing with a $2.2 \mathrm{~ns}$ separation was observed [89]. This separation is 237 times the pulse duration, yet much smaller than the equal-spacing distance in case of harmonic mode-locking. However, the stability of such multi-pulsing has not been reported.

In the process of scaling up DS pulse energy, a new type of stable twin soliton was observed, whose temporal separation is in the tens of ns, very different from any soliton mentioned above. The discovery of this twin soliton and its properties will be described as following. 


\subsection{Experimental setup}

The design of the laser, depicted in Fig. 6.1, was almost as same as the one described in chapter 5, except for adding one piece of PM 085 LMA fiber (50 m) into the cavity. The dispersion of the PLMA fiber was depicted in Fig. 6.2, provided by Nufern. The GVD of the PLMA fiber is $2 / 3$ compared to that of the PM 980 fiber and the core area of the PLMA fiber is 2.6 times larger than that of the PM 980 fiber. Therefore, both GVD and SPM inside the PLMA fiber will be smaller than those if a same length PM980 fiber is used. If one want to scale-up the pulse energy by extending the cavity length, the PLMA fiber would be a good choice.

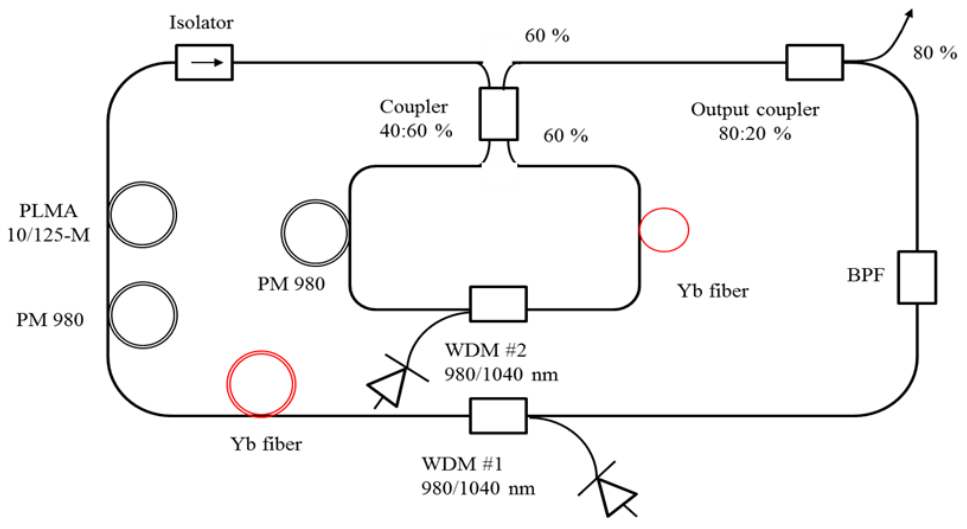

Fig. 6.1 Scheme of an all-fiber all-PM NALM DS fiber laser.

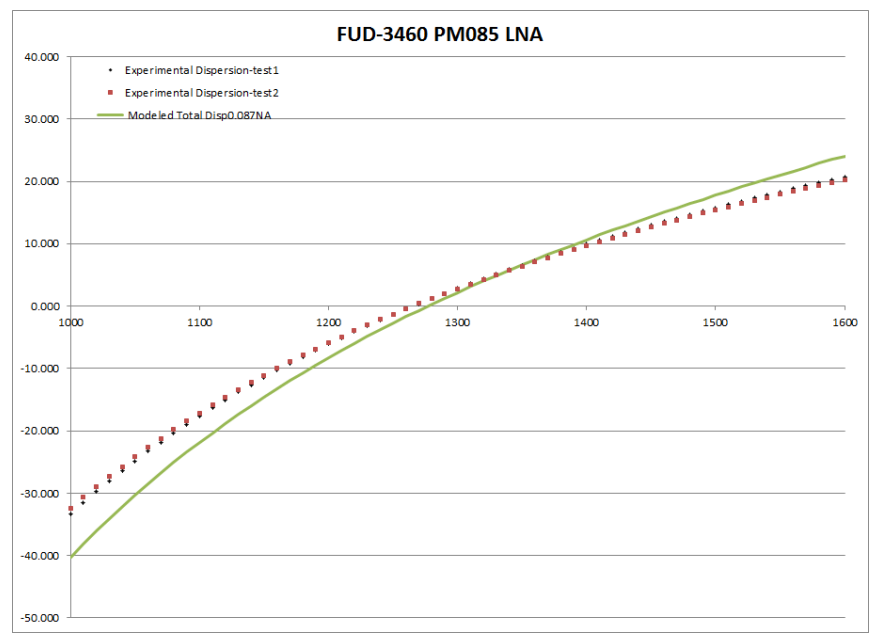

Fig. 6.2 Dispersion map of the PM085 LMA fiber by Nufern 


\subsection{Experimental results}

There are two pumps whose power can be tuned in this laser configuration. One is the pump in the main loop, $\mathrm{P}_{\text {main, }}$ which can change the working regime of the laser. The other is the pump in the NALM loop, $\mathrm{P}_{\mathrm{NALM}}$, which can change the modulation depth of the saturated absorber. Firstly, the PNALM was fixed at 377 $\mathrm{mW}$ and scanned the $\mathrm{P}_{\text {main }}$ to find different working regimes, as shown in Fig. 6.3a. The laser works at $\mathrm{CW}$ regime first when the $\mathrm{P}_{\text {main }}$ is lower than $273 \mathrm{~mW}$. A noise-like pulse will self-start when the $P_{\text {main }}$ exceeds $273 \mathrm{~mW}$. Its spectrum is shown in Fig. 6.4a with a characteristic smooth triangle-shape spectrum peaked at $1030 \mathrm{~nm}$. The spectrum width became broadened with the increase of $\mathrm{P}_{\text {main }}$ (Fig. $6.4 \mathrm{~b})$. The laser will enter into an unstable working regime when the $\mathrm{P}_{\text {main }}$ is between $482 \mathrm{~mW}$ and $542 \mathrm{~mW}$. Such regime is the middle area between noiselike pulses and stable DS. After the $P_{\text {main }}$ exceeds $542 \mathrm{~mW}$, the laser starts to produce stable DS, whose spectrum shows the steeper edges with characteristic ripples on both side of the spectrum (Fig. 6.4c). The changing of the working regime is accompanied by a sudden increase of laser output power as shown in Fig. 6.3a. The laser will not jump out from the DS regime even the $P_{\text {main }}$ reaches its maximum limit $(600 \mathrm{~mW})$. To sum up, when the $\mathrm{P}_{\text {NALM }}$ is fixed at $377 \mathrm{~mW}$, the laser will enter four different regimes $(\mathrm{CW}$ - noise like - unstable - stable DS) with the increase of $P_{\text {main. }}$.

Because the $\mathrm{P}_{\text {main }}$ reached its limit, in order to get higher DS output power, the $\mathrm{P}_{\text {main }}$ was then fixed at $578 \mathrm{~mW}$ and increased the $\mathrm{P}_{\mathrm{NALM}}$. The laser stays at single pulse DS regime when the $\mathrm{P}_{\mathrm{NALM}}$ is lower than $470 \mathrm{~mW}$. Four different pulsebreaking phenomenon can be observed after $\mathrm{P}_{\text {NALM }}$ exceeds this level as shown in Fig. $6.3 \mathrm{~b}$, which will be further described in detail in the following paragraph. The spectrum of such breaking pulses is quite similar to the single DS regime, only with slightly broaden bandwidth due to higher pump power and more characteristic ripples even shown in the bottom of the DS spectrum (Fig. 6.4d). 
The output power of the laser has a sudden increase from $78.5 \mathrm{~mW}$ to $84.4 \mathrm{~mW}$ when the pulse-breaking happens.
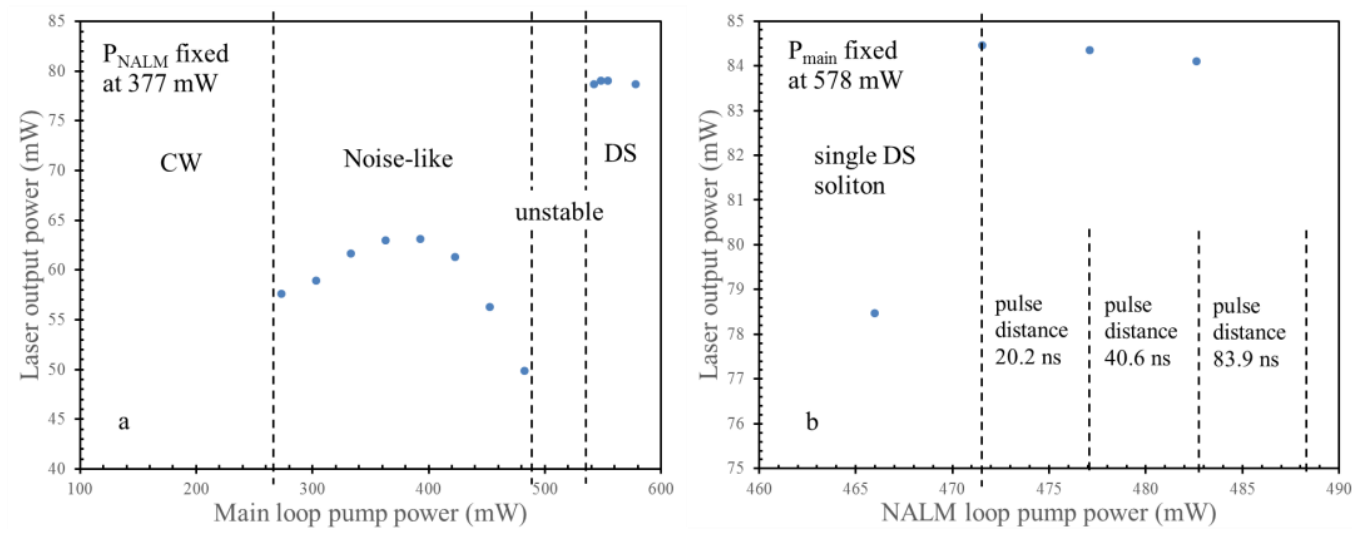

Fig. 6.3 Different working regimes of the NALM mode-locked laser.
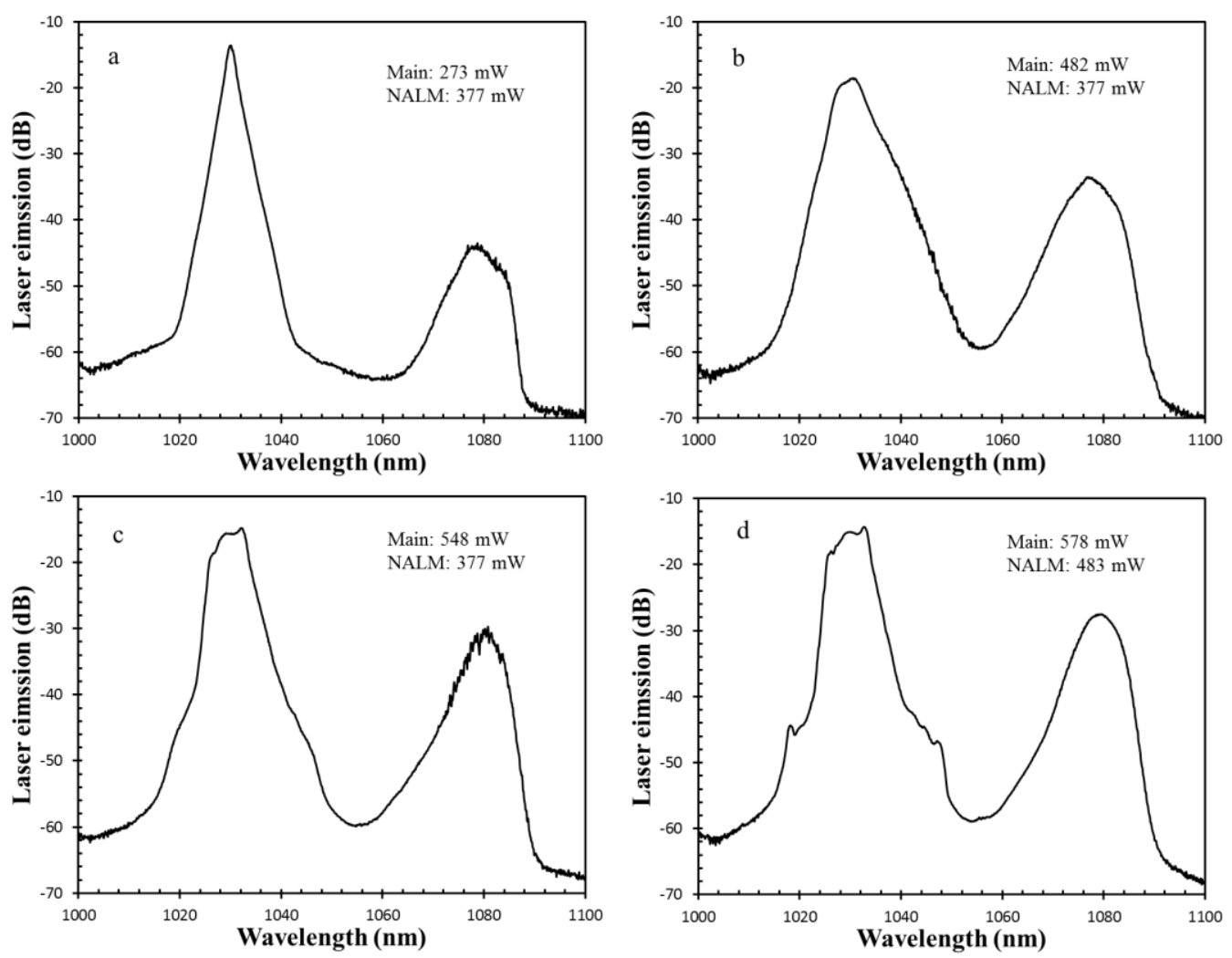

Fig. 6.4 Spectrum evolution of the NALM mode-locked laser.

The mode-locked pulse train in single DS pulse regime is shown in Fig. 6.5a with $\mathrm{P}_{\text {main }}=578 \mathrm{~mW}$ and $\mathrm{P}_{\mathrm{NALM}}=377 \mathrm{~mW}$. The measured pulse spacing of $713 \mathrm{~ns}$ 
corresponds to a repetition rate of $1.41 \mathrm{MHz}$, matching well with the cavity length of $143 \mathrm{~m}$. When the P PALM is increased to $471 \mathrm{~mW}$, a second pulse starts to appear as a shoulder of the original pulse and sweeps away immediately. The distance between this two pulses keeps increasing until it reaches $20.2 \mathrm{~ns}$, where the newly-generated pulse propagates with the same speed as the original pulse and become a stable state (Fig. 6.5b). When the PNALM is further increased to $477 \mathrm{~mW}$, the second pulse starts to move again until it reaches the distance of $40.6 \mathrm{~ns}$, which is another stable state (Fig. 6.5c). Same thing happens, when the $\mathrm{P}_{\mathrm{NALM}}$ is further increased to $483 \mathrm{~mW}$. At this pump level, the distance between the two pulses is increased to $83.9 \mathrm{~ns}$ (Fig. 6.5d). When the PNALM exceeds 487 $\mathrm{mW}$, the second pulse begins to drift away again but this time it would not stop no matter how much the pump power increases. I refer this case as an unstable drifting state. All these four pulse-breaking state have been summarized in Fig. $6.3 b$.
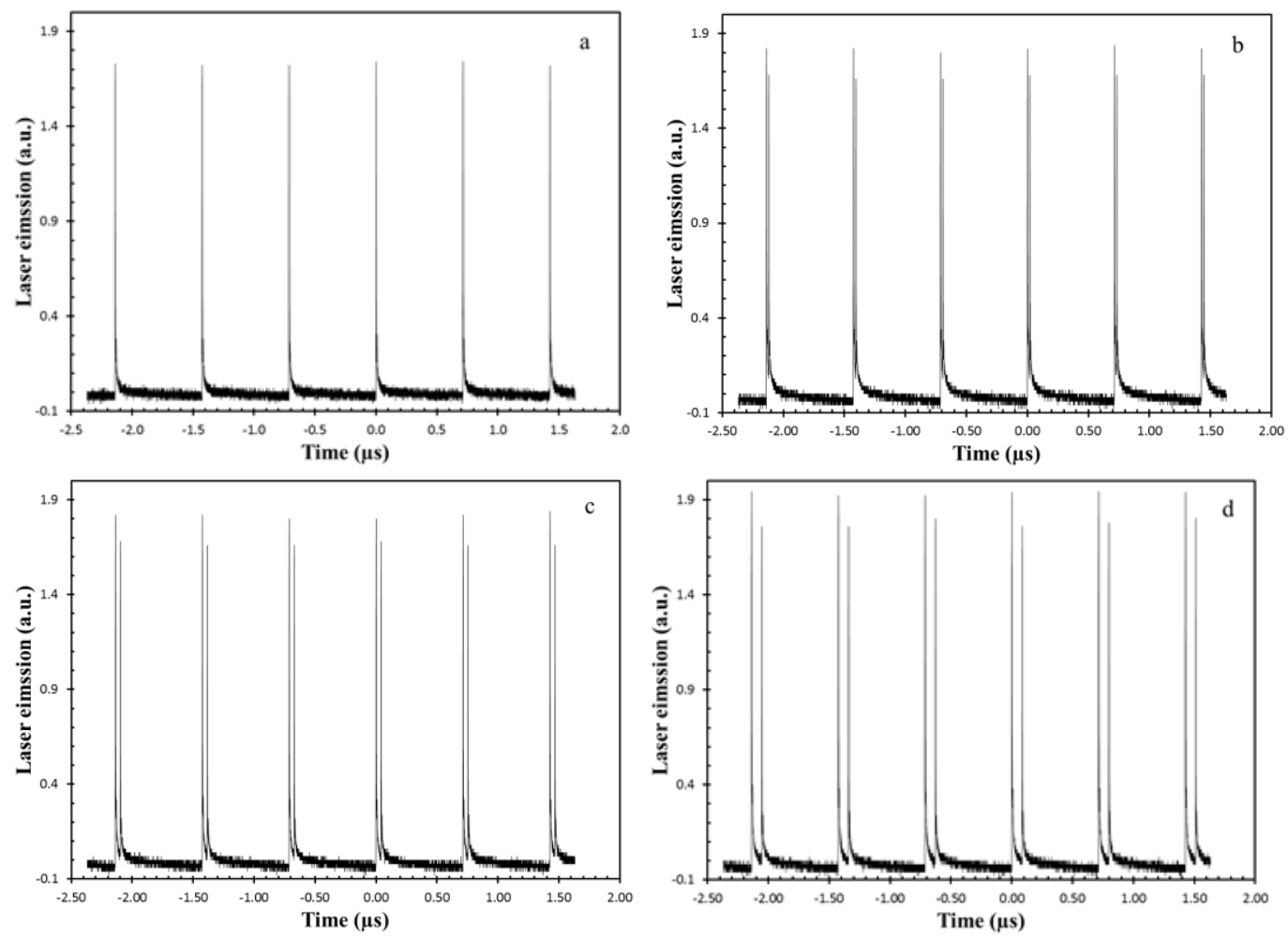

Fig. 6.5 Output pulse train of the NALM mode-locked laser in DS regime with different NALM pump power (a) $377 \mathrm{~mW}$, (b) $471 \mathrm{~mW}$, (c) $477 \mathrm{~mW}$, (d) $483 \mathrm{~mW}$. 

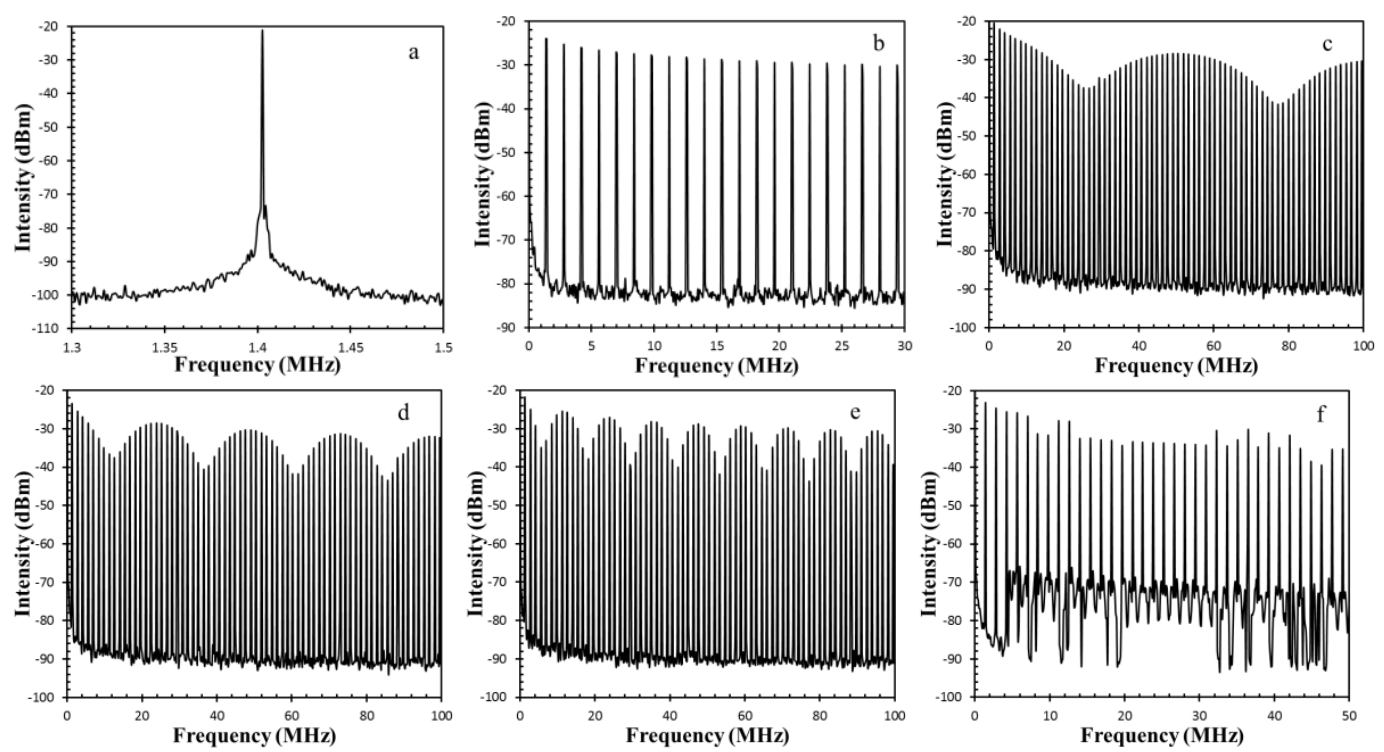

Fig. 6.6 RF spectrum at the fundamental repetition rate in the single pulse DS regime (a), harmonic RF spectrum of the single pulse DS regime (b), harmonic RF spectrum of the multi-pulse DS regime with separating distance of $20.2 \mathrm{~ns}$ (c), $40.6 \mathrm{~ns}$ (d), $83.9 \mathrm{~ns}$ (e), harmonic RF spectrum of the unstable multi-pulsing (f).

The RF spectra for the single pulse DS was measured at a resolution of $10 \mathrm{~Hz}$ for the fundamental frequency, as shown in Fig. 6.6a. The RF spectrum with a high signal-to-noise ratio of $80 \mathrm{~dB}$ and no side-peaks indicates high pulse-to-pulse stability and low amplitude modulation of the DS soliton. The fundamental RF spectra of the three stable pulse-breaking states are very similar to the single pulse results. The difference is their harmonic RF spectra. The harmonic RF spectrum of single DS pulse is shown in Fig. $6 \mathrm{~b}$ in the range of $30 \mathrm{MHz}$ and with a resolution of $1 \mathrm{kHz}$. The harmonic RF spectra of three stable pulse-breaking states are shown in Fig. 6.6c, d, e, respectively, in the range of $100 \mathrm{MHz}$. Periodical modulation with peaks and troughs can be observed in all three situations. The first peak of each spectrum is at $50 \mathrm{MHz}, 25 \mathrm{MHz}$ and $12 \mathrm{MHz}$, corresponding to the breaking distance of $20.2 \mathrm{~ns}, 40.6 \mathrm{~ns}$ and $83.9 \mathrm{~ns}$ respectively. Each spectrum has been measured over 2 hours and no fluctuation has been observed, which proves the high stability of such states. Fig. $6.6 \mathrm{f}$ shows the harmonic RF spectrum of the unstable state when the $\mathrm{P}_{\text {NALM }}$ is over $487 \mathrm{~mW}$. The higher background level and irregular RF peaks indicates the unstable characteristics of this state. 


\subsection{Discussions}

The wave-breaking phenomenon observed here has several unique characteristics: 1) the new-generated pulse has the similar intensity as the original pulse, 2) the distance between the two pulses is tens of ns, 3) when the pump power is adjusted at certain level such breaking states are very stable, and 4) the distance between the two pulses can be step-tuned by $\mathrm{P}_{\mathrm{NALM}}$. Therefore, this twin-soliton is a new type of soliton; unlike soliton molecular whose temporal separation is at the level of ps. It is also not like soliton rains whose breaking pulses have much lower intensity compared to the original pulse and they always keep drifting. It is also not harmonic mode-locking whose pulses are equally spacing.

Noticing that the first stable breaking separation, $20.2 \mathrm{~ns}$, is the round trip time of the NALM loop, this breaking phenomenon can be explained as such: when the pump power is increased, the spectral width and pulse width of the DS are also increased due to SPM, however, limited by the gain bandwidth of Yb. Once the spectral width reaches its limitation, its pulse width is also confined and the corresponding DS pulse is clamped. Therefore, further increasing the pump cannot change the soliton parameters but increases the gain for the dispersive waves. When the dispersive waves become intense, they will form a new soliton. Because of the gain medium depletion, the newly-generated pulse will drift away and tend to form an equal-spacing harmonic mode-locking. However, when the breaking distance reaches $20.5 \mathrm{~ns}$, an interaction between the two pulses happens because of the NALM structure. The leading pulse (original pulse) will enter the NALM loop first. After travelling by $20.2 \mathrm{~ns}$ (NALM loop length is $4.3 \mathrm{~m}$ ), it will come out from the loop. At this time, second pulse is just about to enter the NALM loop. Therefore, the two pulses will interact with each other, resulting in a phase-lock and thus maintain the pulse separation at $20.2 \mathrm{~ns}$. The other two stable breaking distance $40.6 \mathrm{~ns}$ and $83.9 \mathrm{~ns}$ are close to 2 and 4 times of the round-trip time of the NALM (20.2 ns). 


\subsection{Summary}

A new twin-soliton phenomenon in an all normal dispersion mode-locked fiber laser was demonstrated. The DS laser is based on a long-cavity ring structure, which uses a nonlinear amplified loop mirror (NALM) as the stature absorber. 50 nJ single DS pulse operation can be achieved under certain pump power. Further increasing the pump power will lead to three stable twin-soliton states with a temporal separation of $20.2 \mathrm{~ns}, 40.6 \mathrm{~ns}$ and $83.9 \mathrm{~ns}$ respectively. Such twinsoliton can be used in the application like ranging of moving object in $\mu \mathrm{m}$ scales. 


\section{Chapter 7}

\section{SMS fiber filters and lasers}

Single-mode- multimode- single-mode (SMS) fiber filters are the structures which use mode interference to achieve certain filter spectrum. The light from the single-mode fiber couples into many modes in the multimode fiber and the interference of these modes, filtered out by an output single-mode fiber, results in the periodic images of the input field on the output fiber axis. SMS fiber structures have been employed in many applications such as sensors for strain, temperature and refractive index. In this chapter, a new type of SMS filter near 1 um region and its applications, one in a high efficiency $\mathrm{CW}$ fiber lasers and the other in a Q-switched fiber laser were presented.

\subsection{SMS fiber filters}

\subsubsection{Experimental setup}

In an SMS filter, a piece of double cladding LMA fiber of $38 \mathrm{~cm}$ in length, with core/cladding diameters of $20 / 130 \mu \mathrm{m}$, was spliced between two single mode fibers (HI1060, Corning), as shown in Fig. 7.1. The mode-match program of the fusion splicer (Model FSU 995 FA) was used to reduce the splice loss. The total insertion loss of this SMS filter was measured to be at $1.2 \mathrm{~dB}$. One Yb-doped ASE (Amplified Spontaneous Emission) broadband light source from 1000 to $1120 \mathrm{~nm}$ and the other semiconductor light diode source (SLD) covering 900 to

$1030 \mathrm{~nm}$ were used as the light source and an optical spectrum analyzer (Model AQ-6317, Ando) was used to measure the spectrum of the filter at a resolution of $0.1 \mathrm{~nm}$. 


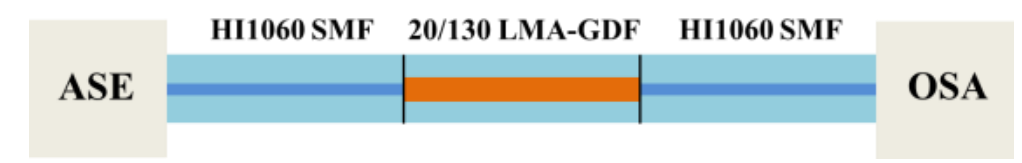

Fig. 7.1 Schematic diagram and the testing layout of SMS fiber filter.

To measure the strain dependence of the SMS transmission spectrum, I placed the SMS filter vertically with one end clamped on to a fixed fiber holder and the other end attached to a weight-carrying basket. I added subsequently the weight of $7.9 \mathrm{~g}$ each and recorded each spectrum. The strain, $\varepsilon$, corresponding to each weight was calculated to be 80.5 micro-strain from the following expression:

$$
\varepsilon=\frac{m g}{A Y}
$$

where $\mathrm{m}$ is the mass of the weight; $\mathrm{g}=9.8 \mathrm{~m} / \mathrm{s}^{2}$ is the acceleration due to gravity; $\mathrm{A}$ is the cross-section area of the fiber cladding and $\mathrm{Y}=72.5 \mathrm{Gpa}$ is the Yonge's module of the silica fiber.

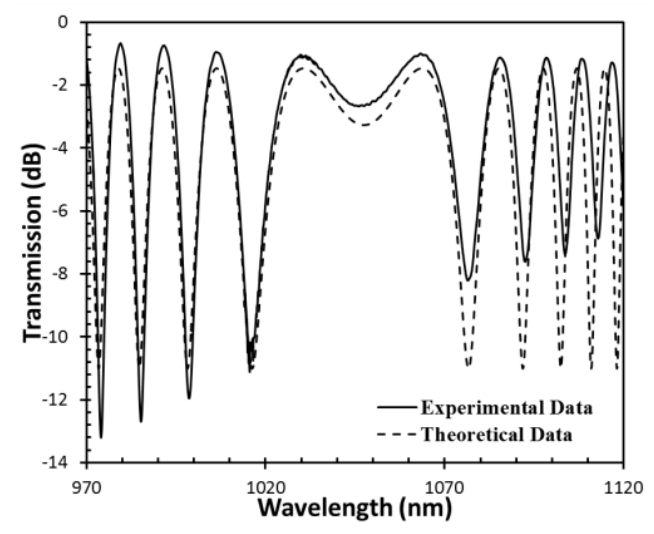

Fig. 7.2 Transmission spectrum from $970 \mathrm{~nm}$ to $1120 \mathrm{~nm}$ without strain (solid line); and simulated spectrum (dashed line).

The temperature dependence of the SMS transmission spectrum was also tested by placing the filter on a metal bar and inserting the structure into a $50 \mathrm{~cm}$ wide convection oven. Two access ports on both side of the oven were used to lead fiber pigtails in and out of the oven. The transmission spectra were measured when the temperature inside the oven became stable. 
The transmission spectrum of this SMS filter in the wavelength range of 970-1120 $\mathrm{nm}$ is plotted in Fig. 7.2, in which, nearly symmetric interference fringes can be observed on the both side of the critical wavelength, $\lambda_{0}$. The dispersion turning wavelength is at $1046 \mathrm{~nm}$, which resulted in a broad trough at this wavelength. For the wavelength shorter than $\lambda_{0}$, the spacing of the interference fringes increase with increasing wavelength. However, for the wavelength longer than $\lambda_{0}$, the fringe spacing decreases with increasing wavelength. The contrast of the interference fringes is about $12 \mathrm{~dB}$ on the short wavelength side and about $7 \mathrm{~dB}$ on the long wavelength side; which is much larger than the fringe contract between 4 to $5 \mathrm{~dB}$, observed in ref. [44] and [45]. Since the amount of light coupled from the single-mode fiber to the $\mathrm{LP}_{01}$ and $\mathrm{LP}_{02}$ mode are not the same, a large difference will lead to the low contrast of the interference fringes. The high contrast observed in Fig. 7.2 shows more even coupling into the two modes in the LMA fiber, which is advantageous for sensing applications. Since the V-number of the multimode fiber is about 6 , sufficiently large for this fiber to support about 10 modes, it is remarkable that the interference of spectrum looks similar to the two beam interference due to the relatively even coupling of light to $\mathrm{LP}_{01}$ and $\mathrm{LP}_{02}$ modes.

\subsubsection{Spectral simulation}

The transmission spectrum in Fig. 7.2 is interpreted as the interference of $\mathrm{LP}_{01}$ and $\mathrm{LP}_{02}$ modes in the core of the LMA fiber. To verify this, the dispersion of the propagation constants, $\beta_{1}$ and $\beta_{2}$ of the two modes was calculated and their difference, $\Delta \beta$, was used to calculate the transmission spectrum. Since material dispersions of the two modes are the same, only waveguide dispersions are considered in obtaining $\Delta \beta$ as a function of wavelength.

If the ratios of optical power transferred to the $\mathrm{LP}_{01}$ and $\mathrm{LP}_{02}$ modes from the lead-in single-mode fiber are $r_{1}$ and $r_{2}$, respectively, assuming only two-mode interference, the light transmitted through the SMS, T, can be written as: 


$$
T=\frac{P_{o u t}}{P_{\text {in }}}=r_{1}^{2}+r_{2}^{2}+2 r_{1} r_{2} \cos (\Delta \beta L)
$$

where $\mathrm{L}$ is the physical length of the LMA fiber. If the $\Delta \beta$ changes with the wavelength, the spacing between the interference peaks would changes correspondingly.

For a step-indexed fiber, the propagation constant for its $\mathrm{m}$-th symmetric mode can be expressed as:

$$
\beta_{0 m}=k_{0}\left[n_{2}^{2}+b_{0 m}\left(n_{1}^{2}-n_{2}^{2}\right)\right]^{1 / 2}
$$

where $\mathrm{m}=0,1,2,3, \ldots$ and $\mathrm{n}_{1}, \mathrm{n}_{2}$ stand for refraction index of core and cladding, respectively. $\mathrm{k}_{0}$ is the free space wavenumber. $\mathrm{b}_{0 \mathrm{~m}}$ is the normalized propagation constant, defined as:

$$
b_{0 m}=1-\frac{u_{0 m}^{2}}{V^{2}}
$$

where $\mathrm{V}$ is the normalized frequency and $\mathrm{u}_{0 \mathrm{~m}}$ is the normalized transverse propagation constant for each $\mathrm{m}$-th symmetric mode. The analytic procedure outlined in ref. [90] yields the normalized transverse propagation constant as a function of $\mathrm{V}$ :

$$
u_{0 m}(V) \approx u_{c, 0 m} \exp \left(\frac{\sin ^{-1}\left(s / u_{c, 0 m}\right)-\sin ^{-1}(s / V)}{s}\right)
$$

where $\mathrm{u}_{\mathrm{c}, 0 \mathrm{~m}}$ is the value of $\mathrm{u}_{0 \mathrm{~m}}$ at cut-off for the mode in question and $\mathrm{s}=\left(\mathrm{u}_{\mathrm{c}, 0 \mathrm{~m}}^{2}-\right.$ $1)^{1 / 2}$. For the $\mathrm{LP}_{01}$ mode, a more careful approximation yields:

$$
u_{01}(V) \approx \frac{(1+\sqrt{2}) V}{1+\left(4+V^{4}\right)^{1 / 4}}
$$

Thus the $\Delta \beta$ between $\mathrm{LP}_{01}$ and $\mathrm{LP}_{02}$ mode can be calculated from Eq. 7.3 to 7.6 as 


$$
\Delta \beta=\beta_{01}-\beta_{02}
$$

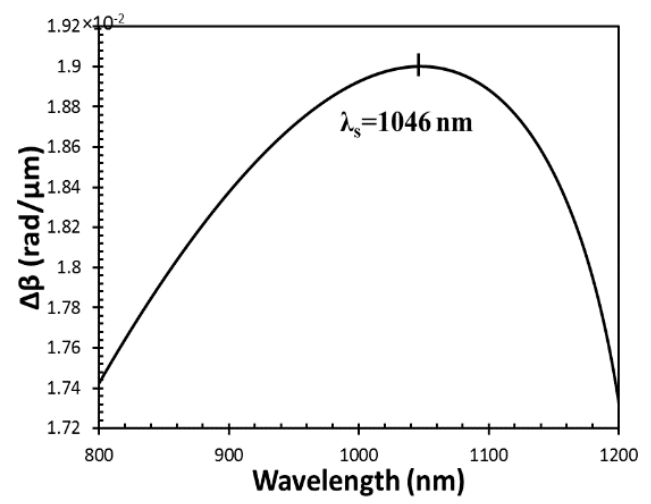

Fig. 7.3 Calculated propagation constant difference, $\Delta \beta$, between the $\mathrm{LP}_{01}$ and $\mathrm{LP}_{02}$ modes as a function of wavelength.

The calculated $\Delta \beta$ vs. $\lambda$ curve is illustrated in Fig. 7.3. The propagation constant difference reaches a maximum value of $0.019 \mathrm{rad} / \mu \mathrm{m}$ at the wavelength of 1046 $\mathrm{nm}$, which is very close to our experiment observation.

Using the calculated dispersion profile of $\Delta \beta$, the transmission spectrum of the SMS filter can be calculated using Eq. (7.2) as shown in Fig. 7.2. The spectrum matches reasonably well with the measured spectrum. The simulation also shows how to shift the critical wavelength; for example, increasing the core diameter of the LMA fiber will shift $\lambda_{0}$ to a longer wavelength.

\subsubsection{Results and discussions}

In order to explore the possibility of applying this filter for sensing application, the strain and temperature dependence of the filter transmission spectra were characterized. The LMA fiber was selected to be $38 \mathrm{~cm}$ in length to accommodate the width of the temperature chamber. The tensile strain was applied to the filter by adding weight to a hook that was attached to one end of the fiber. When the filter is subjected to strain, the spectral shifts were also observed for these interference fringes: the fringes on the right side of the $\lambda_{0}$ show red shift and the fringes on the left side show blue shift. Furthermore, the fringe close to the $\lambda_{0}$ 
shows substantially large shift than those further away from $\lambda_{0}$. The spectral shifts of the first and second peak on the longer wavelength side of the critical wavelength were recorded as a function of strain as shown in Fig. 7.4(a). The first peak is red-shifted at a rate of $3.1 \mathrm{pm} / \mu \varepsilon$ which is 2.6 time higher than what can be obtained from an FBG sensor in a silica fiber [91]. The second peak is also red-shifted, nevertheless, at a lower rate of $1.8 \mathrm{pm} / \mu \varepsilon$. The fact that difference interference peaks shift at different rates could be useful for sensing application.
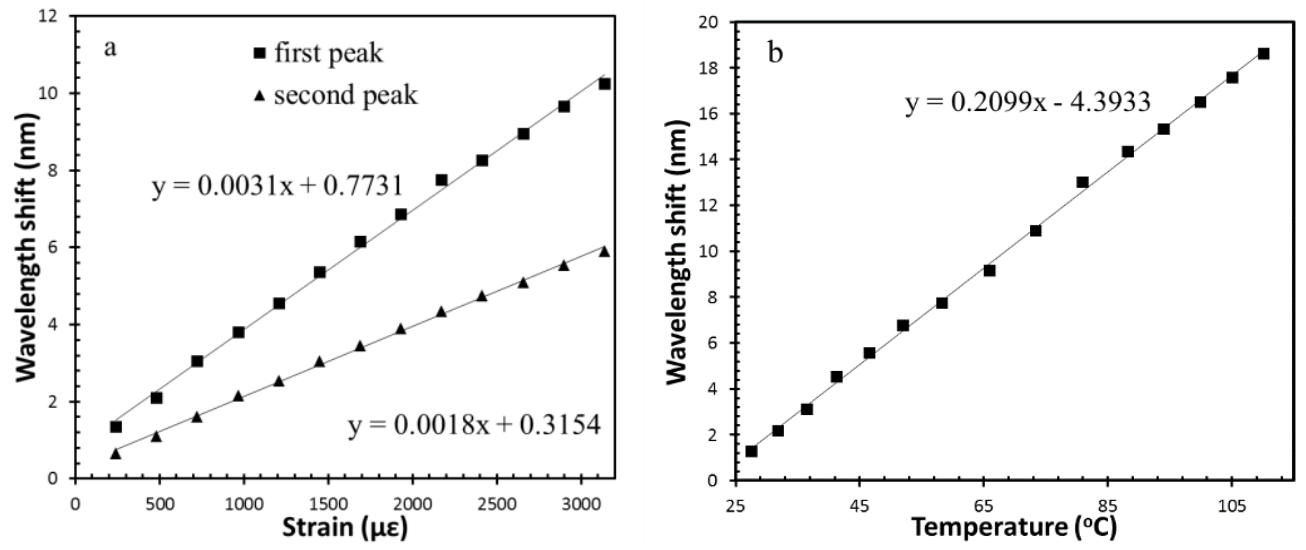

Fig. 7.4 (a) Wavelength shift as a function of strain; and (b) Wavelength shift as a function of temperature.

The transmission spectra measured at two different strain levels were shown in Fig. 7.5(a), in which, the transmission and bandwidth of the broad peak/trough vary with the strain, mainly caused by the phase changing when the length of the LMA fiber was extended by the strain. The transmission at the critical wavelength was measured as a function of strain which fits very well with a sinusoidal function, as shown in Fig. 7.5(b). This property can be used to make a variable optical coupler or variable optical attenuator (VOA) near the wavelength $\lambda_{0}$. A rectangular-shaped pass band with a broad bandwidth of $\sim 20 \mathrm{~nm}$ was obtained at a strain value of $1450 \mu \varepsilon$. The pass band could also be obtained by adjusting the LMA length without strain. Since this filter has an open band within the Ybdoped fiber emission wavelength and has a fiber output, it can be used directly to vary the output power of an $\mathrm{Yb}$-doped fiber laser. 

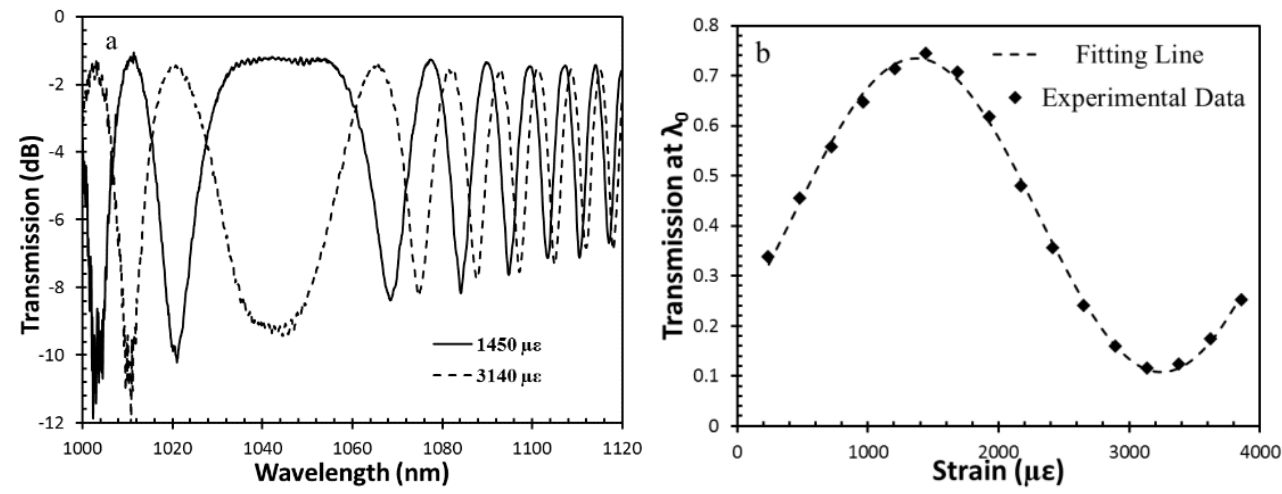

Fig. 7.5 Transmission spectra at two different strain level; and (b) the transmission at the critical wavelength as a function of applied strain.

The same filter was then placed inside a temperature chamber to characterize its temperature response. As shown in Fig. 7.4(b), the first interference peak on the long wavelength side of $\lambda_{0}$ is red-shifted at a rate of $0.21 \mathrm{~nm} / \mathrm{C}$, which is about 20 times higher than the temperature shift of an FBG in C-band [91]. This result shows that this SMS filter can be used as a very sensitive temperature sensor.

\subsection{High efficiency SMS fiber laser with diffraction limited beam output}

In this section, I present the design and test results of an $\mathrm{Yb}$-doped fiber laser operating at $1088 \mathrm{~nm}$. Both ends of the LMA fiber were spliced to the singlemode double-cladding fibers to form an SMS structure. The laser is in all-fiber configuration with two fiber Bragg gratings (FBGs) inscribed onto the two pieces of SMFs respectively to form a laser cavity. Using a $976 \mathrm{~nm}$ laser diode as pump source, output power up to $38.5 \mathrm{~W}$ was generated with a slope efficiency of $70 \%$. Output beam quality was measured with a laser beam profiler and proved to be in a single-transverse-mode. The purpose of this work is to achieve high efficiency $\mathrm{CW}$ fiber laser while still maintaining diffraction limited beam output.

\subsubsection{Experiments}

In an LMA double-cladding fiber, the cladding absorption of the pump power will increase with the diameter of core. For example, the cladding absorption can 
reach $\sim 10 \mathrm{~dB} / \mathrm{m}$ at $976 \mathrm{~nm}$ in a $20 / 130 \mu \mathrm{m}$ core/cladding diameter fiber which is much higher than the cladding absorption in a $6 / 125 \mu \mathrm{m}$ fiber $(\sim 1.7 \mathrm{~dB} / \mathrm{m})$. However, even with such a high absorption, the gain fiber of a few meters is still required for efficient absorption of pump light. With the length of the multimode fiber extended to about 1 to $2 \mathrm{~m}$ long in an SMS filter with a circular-core LMA, our experiment shows that the periodic high transmission peaks can still be observed. An example of such spectrum is shown in Fig. 7.6(a) for an SMS filter with a 25/125 $\mu$ m LMA fiber of 1.35 meter long spliced between two Hi-1060 single-mode fibers, in which, transmission peaks are spaced at every $1.9 \mathrm{~nm}$.
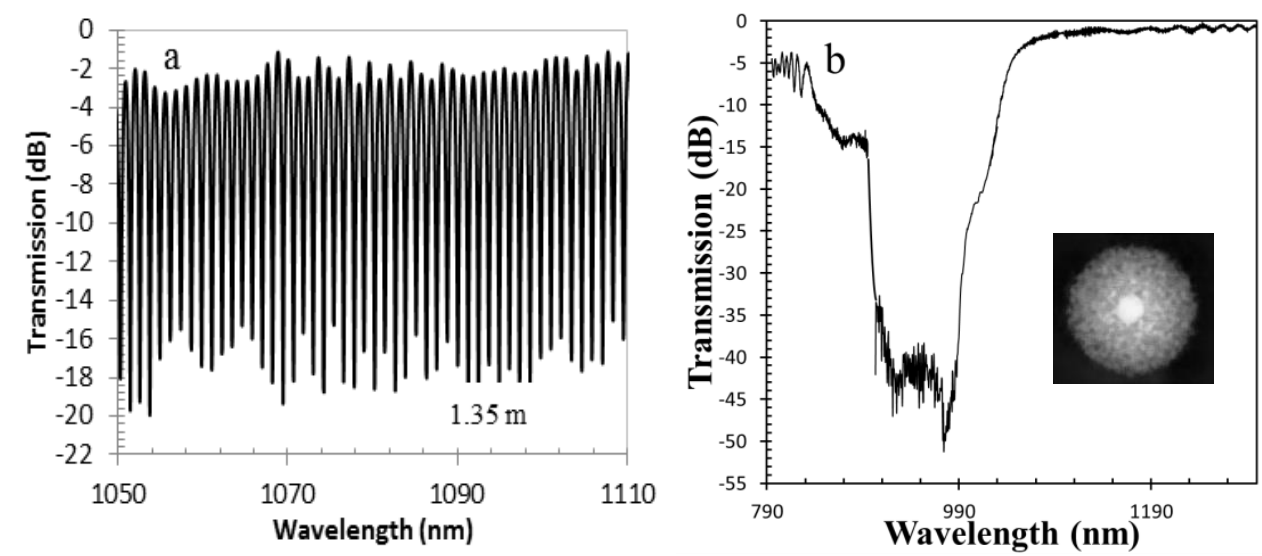

Fig. 7.6 Transmission spectrum of the SMS filter with (a) a passive multimode fiber with a circular cladding; and (b) a gain fiber with an octagonal cladding. The insert shows the photo of the gain fiber core.

The fiber laser consists of an Yb-doped double-cladding fiber of $1.5 \mathrm{~m}$ long spliced between two single-mode fibers (Nufern, SM-GDF-10/125) to form an SMS filter as shown in Fig. 7.7. The core and cladding diameters of the gain fiber are 20 and $130 \mu \mathrm{m}$ respectively. The $\mathrm{V}$-number of the core is 4.36 , which allows about 6 modes to propagate in the core. The cladding has an octagonal shape whose cladding absorption was measured to be $3.3 \mathrm{~dB} / \mathrm{m}$ at $915 \mathrm{~nm}$; therefore the cladding absorption at $976 \mathrm{~nm}$ is estimated to be $\sim 10 \mathrm{~dB} / \mathrm{m}$. A highly reflective FBG with a center wavelength at $1088.8 \mathrm{~nm}$ and a reflectivity of larger than $99 \%$ was written in one of the SMFs. A wavelength matching output coupler (OC) FBG with a reflectivity of $16 \%$ was written in the other SMF to form a laser 
cavity. A $976 \mathrm{~nm}$ diode with a multimode fiber of $105 / 125 \mu \mathrm{m}$ core/cladding diameters as an output fiber was used as a pump source which was spliced to the pigtail of the highly reflective (HR) FBG. Thus the laser was constructed in an all-fiber configuration with all joints fusion spliced. The output coupler FBG was spliced to an optical cable with an APC connector, made with the same 10/125 $\mu \mathrm{m}$ core/cladding diameter fiber. The residual pump power was stripped by coating the splice point with a high index coating.

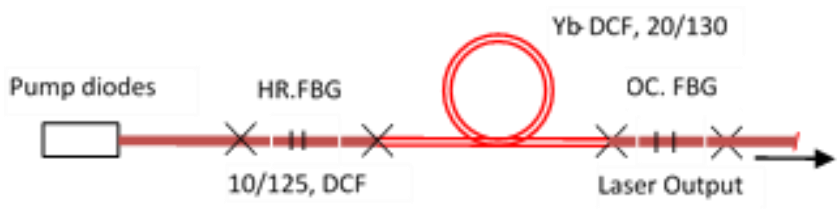

Fig. 7.7 Schematic diagram of the SMS fiber laser.

Transmission spectra were measured with an optical spectrum analyzer (Ando, Model: AQ-6317) at a resolution of $0.1 \mathrm{~nm}$. The output laser beam quality was measured by a beam profiler (Thorlabs, Model: BP109-IR).

The transmission spectrum of the SMS laser (without grating) was measured as shown in Fig.7.6 (b). Instead of interference fringes with a contrast of $\sim 16 \mathrm{~dB}$, shown in Fig. 7.6(a), a broad absorption spectrum of $\mathrm{Yb}$ from 850 to $1050 \mathrm{~nm}$ dominates the spectrum. The interference fringes with a contrast of $4 \mathrm{~dB}$ were observed on the short wavelength side of the Yb-absorption and the fringes with a low contrast of $\sim 1 \mathrm{~dB}$ from 1150 to $1190 \mathrm{~nm}$ were also observed. The interference fringes are still present; the lack of high contrast on the longer wavelength side could be attributed to the octagonal cladding shape, which might also modify the shape of core. The insert in Fig. 7.6(b) shows a photo of the $20 \mu \mathrm{m}$ core which exhibits a slightly deformed circular shape, especially on the left side. However a relative flat transmission spectral region from 1050 to $1100 \mathrm{~nm}$ should benefit the $\mathrm{Yb}$-doped fiber lasers design, because any wavelength in this region could be selected as the lasing wavelength. There is no stringent requirement to match the 
lasing wavelength to a transmission peak. At the lasing wavelength of $1088 \mathrm{~nm}$ the insertion loss of the SMS filter is about $1.6 \mathrm{~dB}$.

\subsubsection{Results and discussions}

The power conversion efficiency of the SMS fiber laser was characterized first. The pump diode was not wavelength stabilized so its wavelength shifted with temperature at a rate of $0.35 \mathrm{~nm} / \mathrm{C}$, which led to a low efficiency when the pump power was below $35 \mathrm{~W}$. However as the pump power increased from $35 \mathrm{~W}$ to $70 \mathrm{~W}$, the pump wavelength moved close to $976 \mathrm{~nm}$, a slope efficiency of $70 \%$ was obtained as shown in Fig. 7.8(a). This efficiency is very close to the efficiency of a cw fiber laser without SMS structure. An output power of $38.5 \mathrm{~W}$ was obtained at a pump power of $70 \mathrm{~W}$, which is much higher than the output power reported in [53].
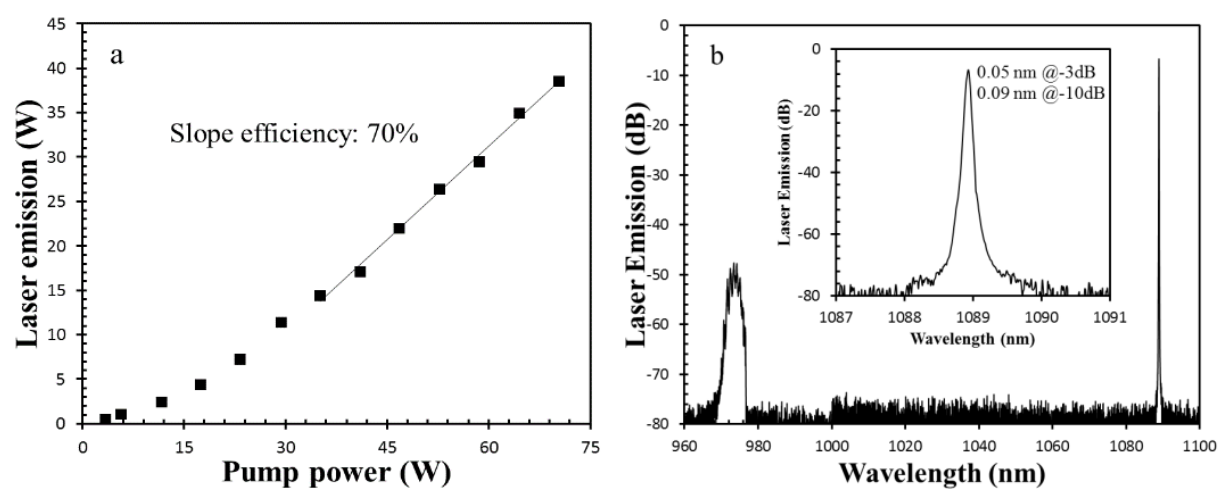

Fig. 7.8 (a) Laser output power as a function of pump power; and (b) Laser emission spectrum with output power at $38.5 \mathrm{~W}$.

The spectrum of the laser output was measured by coupling the laser beam into an APC connector of an optical jump cable with a lens. Amount of power coupled in can be reduced by defocusing the lens. The APC connector on the other end of the cable is connected to an OSA for spectral measurement. As shown in Fig. 7.8(b), the laser emission showed an excellent optical signal to noise ratio of over $70 \mathrm{~dB}$ at $38.5 \mathrm{~W}$ of output power. At that output power, the pump wavelength shifted to the peak absorption of $\mathrm{Yb}$-ion at $976 \mathrm{~nm}$ with its peak at about $40 \mathrm{~dB}$ below the 
laser emission. The laser emission also exhibited a very narrow linewidth of 0.05 $\mathrm{nm}$ at $-3 \mathrm{~dB}$, shown as the inset in Fig. 7.8(b). The narrow linewidth was attributed to the large core size and relatively short cavity length that significantly reduced the laser line broadening caused by self-phase modulation.

An important feature of this design is to retain the high beam quality at its output. The output beam was characterized by a laser beam profiler. Fig. 7.9 shows a 3-D output laser beam profile measured at the output power of $38.5 \mathrm{~W}$. The plots on the left part of the figure show the profiles of the cross sections in $\mathrm{x}$ and $\mathrm{y}$ direction and associated Gaussian fits respectively. As can be seen, the beam profiles exhibited near Gaussian distributions, which proved the high beam quality of the SMS laser output.

In order to prove that the SMS design is essential to the high beam quality, the beam quality of the laser emission directly from the $20 / 130 \mu \mathrm{m}$ gain fiber was also measured. The SMF with the output coupler FBG was removed and the end of the 20/130 gain fiber was cleaved at $90^{\circ}$ so its flat end face with a $4 \%$ reflectivity served as an output coupler. The deterioration of beam quality of this multimode fiber laser is very obvious as shown in Fig. 7.10. The center of the beam in $\mathrm{x}$-direction becomes flat-top with interference ripples, indicating the existence of more than one mode and the interference between the modes.
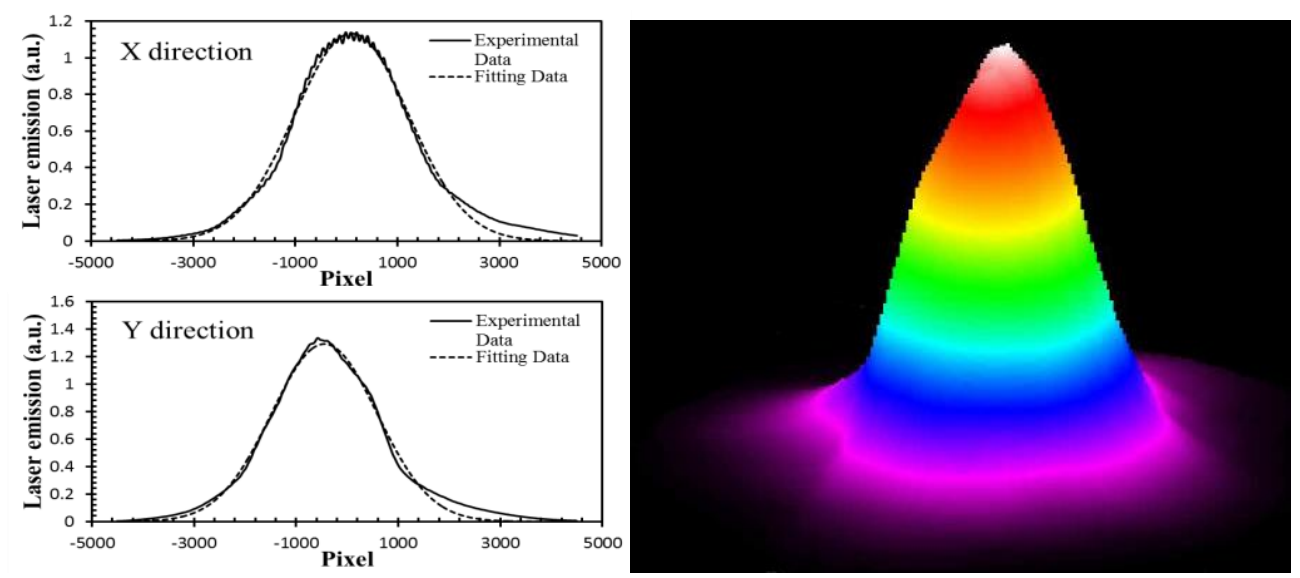

Fig. 7.9 Output laser beam quality of SMS laser measured by beam profile at the output power of $38.5 \mathrm{~W}$. 

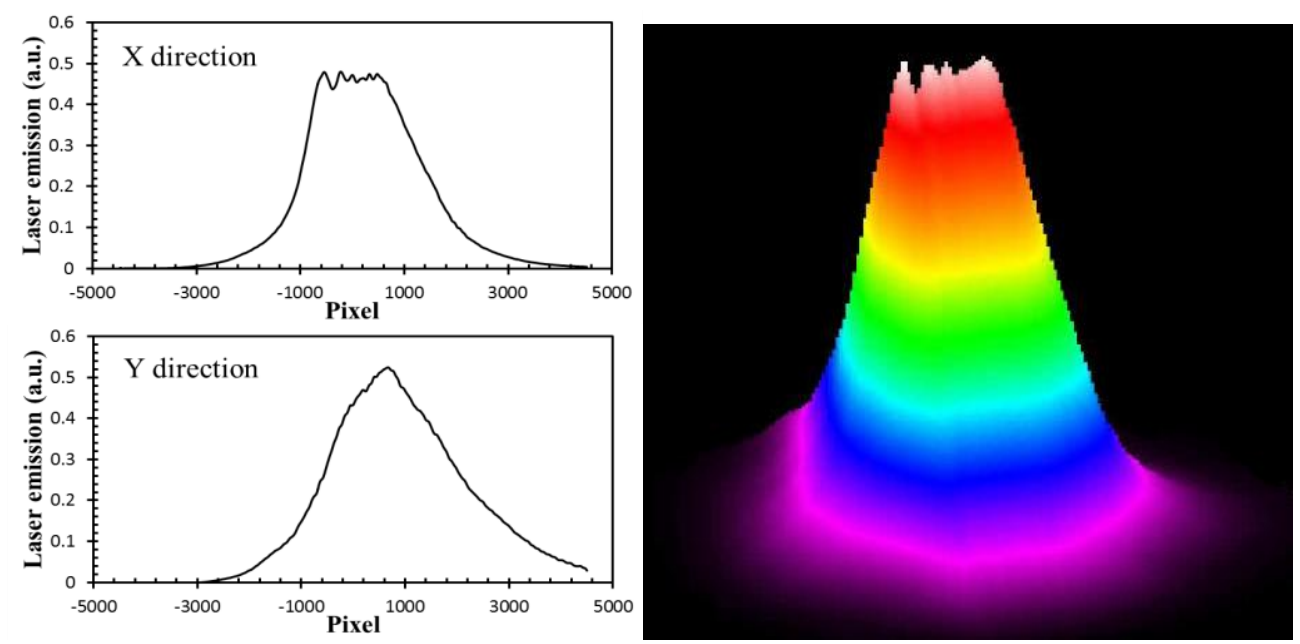

Fig. 7.10 Output laser beam profile from the multimode fiber at the output power of $34.1 \mathrm{~W}$.

In order to evaluate the effect of the SMS structure on the performance of the laser, the stability of the laser output power was measured over 30 minutes. The laser exhibited excellent power stability with a power variation of less than $1 \%$ in standard deviation, as shown in Fig. 7.11.

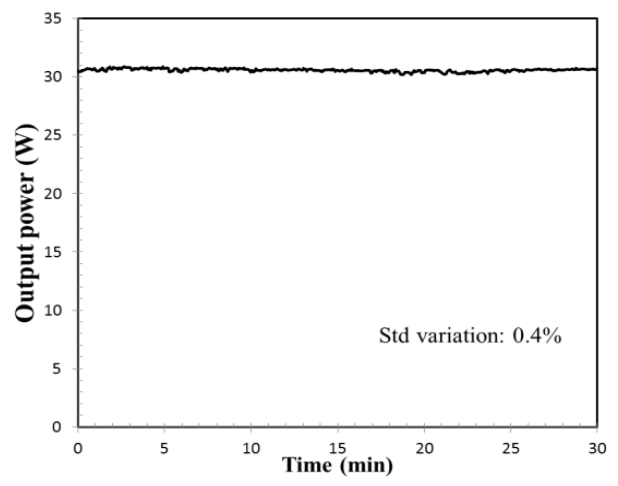

Fig. 7.11 Output power stability of the laser.

\subsection{SMS Q-switched fiber lasers for inhibiting nonlinear effect}

Q-switched fiber lasers are important in laser material processing, second or third harmonic generation and other applications because of its high peak power and high energy output $[92,93]$. Among many designs of Q-switched Yb-doped fiber lasers, a passively Q-switched laser in an all-fiber configuration is particularly interesting because of its robust structure, stable output, compact size and 
maintenance-free operation. Significant progress has been made in the development of this type of laser, especially in scaling up its peak power, particularly with $\mathrm{Yb}$-doped gain fiber. $\mathrm{Hu}$ et al. reported recently an all-fiber passively Q-switched Yb-doped fiber laser with Sm-doped fiber as a saturable absorber [94]. The laser generated $70 \mu \mathrm{J}, 41 \mathrm{~ns}$ output pulses with a peak power of $1.7 \mathrm{~kW}$. However both stimulated Brillouin scattering (SBS) and stimulated Raman scattering (SRS) were observed in the laser output spectra, in which, the broad SRS emission diverted substantial amount of the laser emission to unwanted longer wavelength at $57 \mathrm{~nm}$ away from the laser wavelength. Upadhyaya et al. reported a Q-switched 10- $\mu \mathrm{m}$ core Yb-doped fiber laser with a peak power of $1.3 \mathrm{~kW}$ [95]. An SRS emission peak, shifted by $56 \mathrm{~nm}$ from the laser emission was observed. SRS was also observed in high power fiber amplifiers. Zhao et al. demonstrated a $1 \mathrm{MW}$ peak power, sub-nanosecond master oscillator power amplifier [96]. A distinctive Raman peak was observed in its output spectrum. Malinowski et al. demonstrated an all-fiber pulse laser system with a seed laser and three cascading amplifiers to achieve $180 \mathrm{~kW}$ peak power and $265 \mathrm{~W}$ average power [97]. As much as $18 \%$ of the output was diverted to the Raman emission with a delivery fiber of $1.7 \mathrm{~m}$ long. High peak power and short pulse duration can also lead to wide self-phase modulation (SPM) spectra as observed by LeHneis et al. [98] in a passively Q-switched Nd: YVVO4 microchip laser system, in which, the compressed pulses of $5.9 \mathrm{ps}$ in a single-mode fiber of only $0.3 \mathrm{~m}$ in length lead to SPM broadening as much as $40 \mathrm{~nm}$ (at $-20 \mathrm{~dB}$ ). These examples show that though the progress has been made, researchers in the design of the state-of-the-art high pulse energy and peak power Q-switched laser still face the challenge of overcoming SRS which diverts the laser emission to unwanted Raman emission at the longer wavelength and SPM which significantly broadens the laser emission linewidth. SBS also affects the power scaling up, nevertheless only to the lasers with a very narrow linewidth. For an all-fiber Qswitched laser, because of its sub-nanometer linewidth due to many longitudinal 
modes in cavity, SPM and SRS are the main obstacles to overcome in order to scale up the pulse energy and peak power [99].

One solution to suppress nonlinear effects is to use a large-mode-area (LMA) fiber since the threshold of SPM and SRS is inversely proportional to the LMA [100]. Although SMS structured laser can maintain good beam quality as described in section 7.2, its capacity of suppress nonlinear effects was not demonstrated due to the low average power.

In this section, the design and performance of a Q-switched $\mathrm{Yb}$-doped fiber laser in an SMS structure with a peak output power close to $1 \mathrm{~kW}$ was presented. The laser generated $92 \mu \mathrm{J}, 100 \mathrm{~ns}$ Q-switched pulses at $100 \mathrm{kHz}$. The output spectra of this laser were compared with those of another Q-switched Yb-doped fiber laser with similar output peak power, but not in an SMS structure. The results showed that the Q-switched laser in SMS structure completely inhibited the SRS and significantly reduced SPM.

\subsubsection{Experiments}

The Q-switched fiber assembled in an SMS configuration is shown in Fig. 7.12, in which, a $3 \mathrm{~m}$ long gain fiber with a core and a cladding diameter of 20 and 130 $\mu \mathrm{m}$ respectively, is spliced between two pieces of single-mode fibers with a core diameter of $10 \mu \mathrm{m}$. The $\mathrm{V}$-number of the gain fiber is 4.36, which allows about 6 modes to propagate in the core. The cladding has an octagonal shape whose cladding absorption was measured to be $3.3 \mathrm{~dB} / \mathrm{m}$ at $915 \mathrm{~nm}$; therefore the cladding absorption at $976 \mathrm{~nm}$ is estimated to be $\sim 10 \mathrm{~dB} / \mathrm{m}$. The single-mode fiber on the left of the gain fiber has an FBG of 99\% reflection at $1064 \mathrm{~nm}$ inscribed in it. The left end of the highly reflective FBG is spliced to the multimode output fiber of a $976 \mathrm{~nm}$ pump diode, used as a pump source. The right end of the gain fiber is spliced to a piece of $1.2 \mathrm{~m}$ long $\mathrm{Yb}$-doped fiber with core/cladding diameters of $10 / 130 \mu \mathrm{m}$ which serves as a saturable absorber (SA). These three fibers were spliced concentrically using a mode-matching program to 
reduce the splice loss. The total insertion loss of the SMS structure was $1.8 \mathrm{~dB}$ measured at $1090 \mathrm{~nm}$. The right end of the SA fiber is spliced to a single-mode fiber of $10 / 125 \mu \mathrm{m}$ core/cladding diameters with a low reflective FBG of $10 \%$ reflection inscribed in its core which is used as an output coupler. An anglepolished connector with a pigtail made with the same fiber as the output FBG fiber is used for the laser output. Its splicing point to the output FBG was coated with a high-index coating to remove residual pump light in the cladding. The Qswitched fiber laser, thus built, is in all-fiber configuration with all components spliced together.

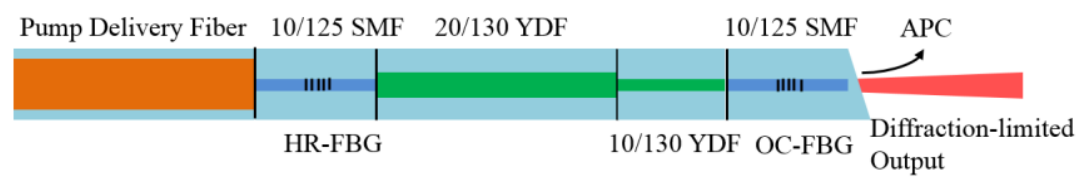

Fig. 7.12 Schematic diagram of the Q-switched SMS fiber laser.

To measure the laser emission spectrum or Q-switched pulse train, the laser output was imaged, by a lens with a $62 \mathrm{~mm}$ long focal length, onto an anglepolished connector of a patch cord. The other end of the patch cord can be inserted into either a photodiode (NewFocus model: 1811) or an optical spectral analyzer (ANDO, model: AQ6317). The amount of light coupled into the patch cord can be reduced by inserting a neutral density filter in the optical path or defocusing the beam.

\subsubsection{Results and discussions}

The mechanism of Q-switching using the same $\mathrm{Yb}$-doped gain fibers of different core diameters is described in ref. [102, 103]. In this laser the Yb-doped fibers of a 20 and $10 \mu \mathrm{m}$ diameter cores are used as the gain fiber and SA fiber respectively. When pump power is absorbed in the gain fiber, the amplified spontaneous emission (ASE) starts to build up. Nevertheless the high loss of the SA in the cavity prevents the lasing. When ASE reaches a sufficiently high level to bleach the SA, the sudden increase of Q-factor in the cavity initiates the Q- 
switch process since the higher population inversion has already established in the gain fiber. The Q-switched pulse depletes population inversion which returns the cavity to an absorbing state. A larger diameter ratio between the gain fiber and the SA fiber allows more energy stored in the gain fiber before Q-switching. In order to assess the capacity of the SMS structure on suppressing SPM and SRS, the performance of the laser was characterized first.
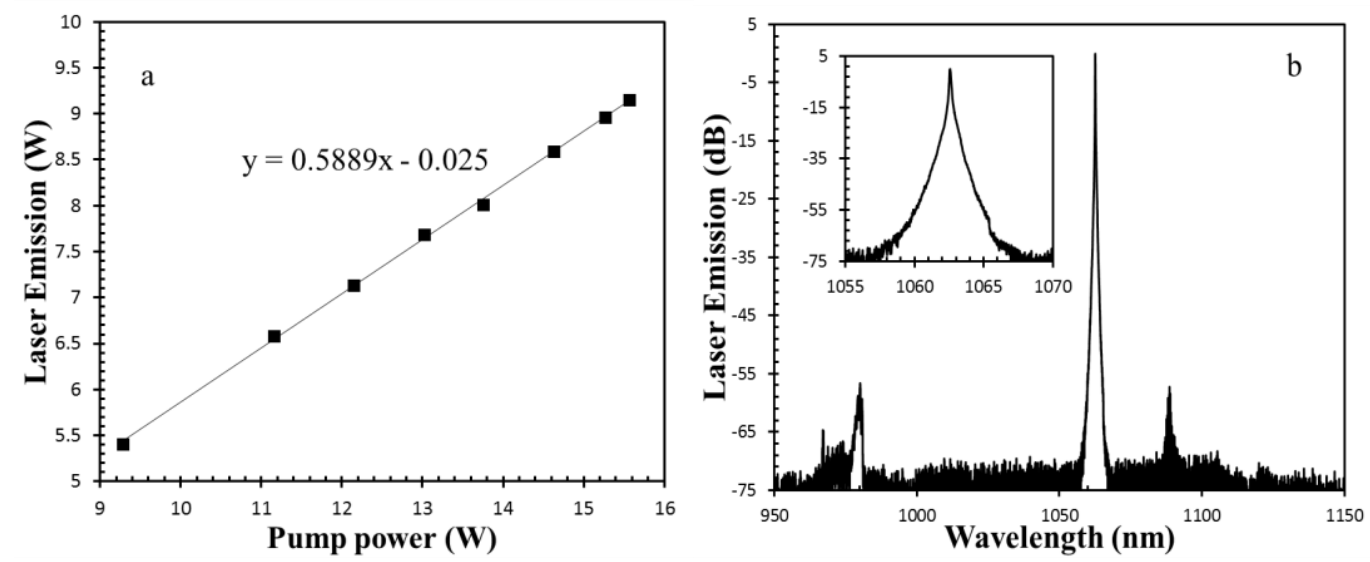

Fig. 7.13 (a) Average output power versus average pump power; (b) Emission spectrum of the laser at $100 \mathrm{kHz}$ in $200 \mathrm{~nm}$ span with the inset showing the spectral detail.

The average output power of the laser was measured as a function of the average pump power and the result was plotted in Fig. 7.13 (a). At $100 \mathrm{kHz}$, the average output power of $9.15 \mathrm{~W}$ was obtained that gave the energy per pulse of $92 \mu \mathrm{J}$. The least-square fitting gave a slope efficiency of $59 \%$ which was almost the same as a Q-switched laser without an SMS structure. The $976 \mathrm{~nm}$ pump diodes were driven by a pulsed current source to suppress relaxation oscillation pulses [104]. At a repetition rate of $100 \mathrm{kHz}$, the pulse width of the driving current was reduced to $1.8 \mu$ s to prevent the start of the relaxation pulse. The laser emission spectrum was measured at the average output power of 9.15W and is plotted in Fig. 7.13(b), in which, the $1064 \mathrm{~nm}$ laser emission shows an excellent optical signal to noise ratio of $70 \mathrm{~dB}$. The residual power at $976 \mathrm{~nm}$ can still be seen; nevertheless at $55 \mathrm{~dB}$ below the laser emission, which confirms the high cladding absorption even with only 3 meters of the gain fiber. 
The Q-switched laser pulse train and a single pulse were shown in Fig. 7.14 (a) and (b) respectively. The pulse shape is asymmetrical with a steep rising edge and a slower tailing edge which is typical for the Q-switched pulses. The full-width at half maximum (FWHM) of the pulse is $100 \mathrm{~ns}$ which gives a peak power of 920 W. The peak power is more than six times higher than that produced by the Qswitched laser with a Sm-doped SA reported in [104].
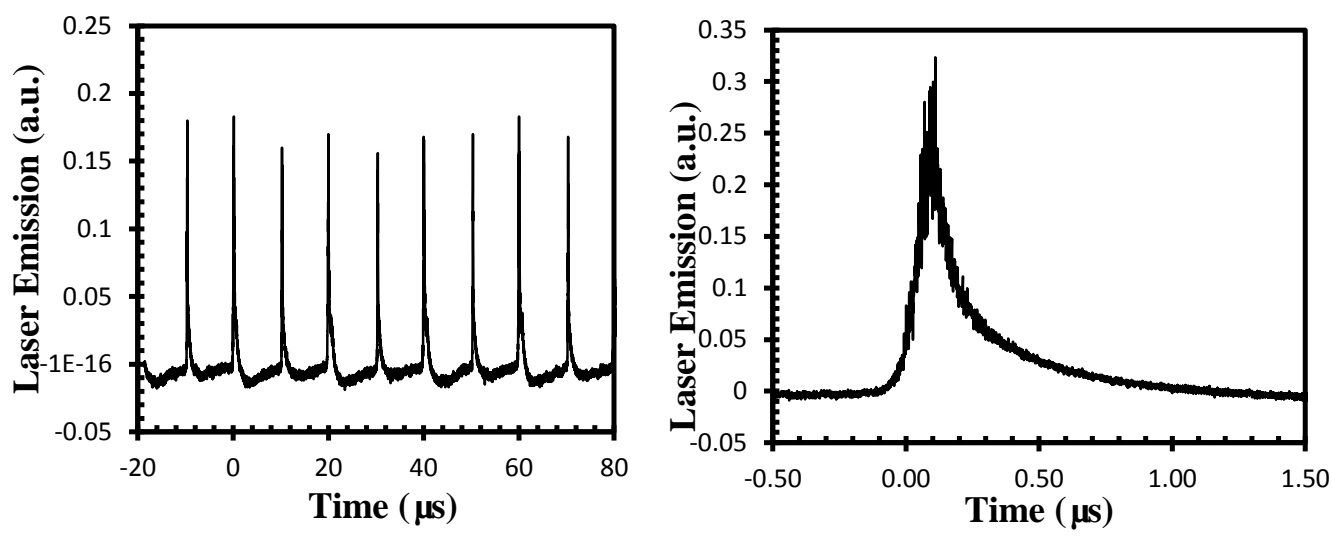

Fig. 7.14 (a) Oscilloscope trace of the Q-switching pulses at $100 \mathrm{kHz}$ rate; (b) a single pulse with $92 \mu \mathrm{J}$ energy and a $100 \mathrm{~ns}$ (FWHM) pulse width.

The linewidth broadening of the laser with the increase of peak power can be seen in Fig. 7.15(a) from the spectra measured at different peak power levels. The linewidth started and remained at $\sim 0.08 \mathrm{~nm}$ until the peak power reached $500 \mathrm{~W}$; then it increases almost linearly to $0.12 \mathrm{~nm}$ at a peak power of $920 \mathrm{~W}$, as shown in Fig. 7.15(b).

In order to assess the effectiveness of the SMS structure on the inhibition of nonlinear effects, the emission spectrum of this laser with that of another laser with a similar output peak power, but, not in SMS structure ware compared. The reference laser was built using the components similar to those used in the SMS laser as shown in Fig.7.12 with the exception of the gain fiber - a $7 \mathrm{~m}$ long $\mathrm{Yb}$ doped gain fiber with core/cladding diameters of $10 / 130 \mu \mathrm{m}$ was used to replace the $20 / 130 \mu \mathrm{m}$ core/cladding Yb-doped gain fiber. Thus this fiber laser is not an SMS structure. A longer gain fiber is necessary to fully absorb the pump power 
since a smaller core leads to a low cladding pump absorption of $3.9 \mathrm{~dB} / \mathrm{m}$ at 976 $\mathrm{nm}$. The reference laser emitted $7.97 \mathrm{~W}$ of average power at a repetition rate of $100 \mathrm{kHz}$ limited by the pump power. The pulse width of the laser was $100 \mathrm{~ns}$ that gave a peak power of $800 \mathrm{~W}$.
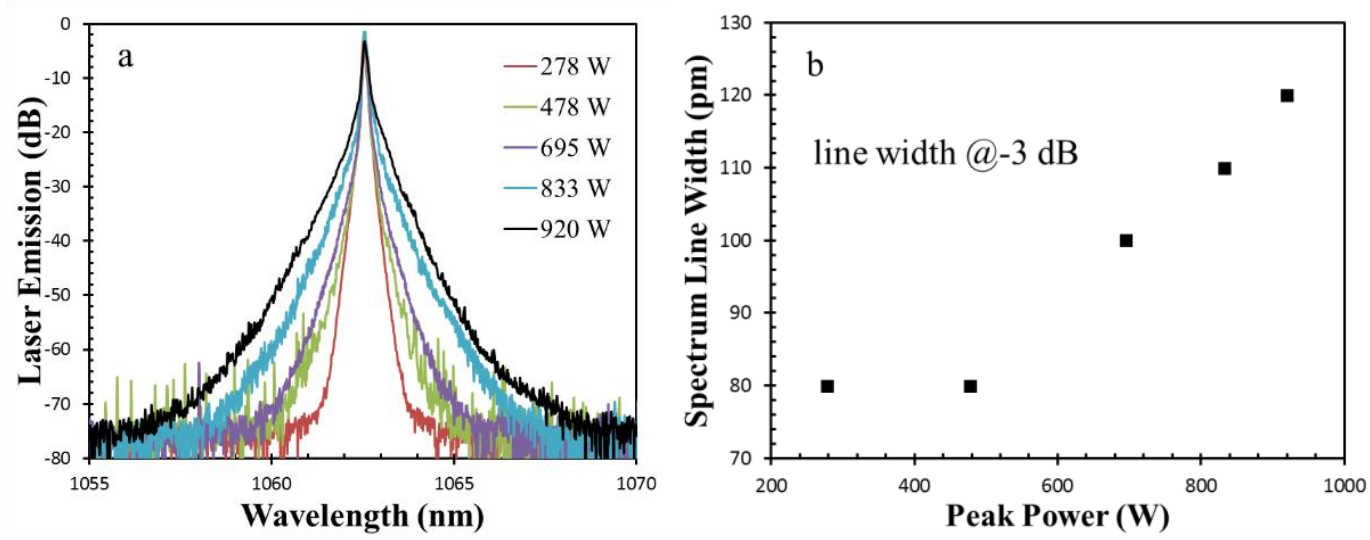

Fig. 7.15 (a) Laser spectra at different peak power; (b) Spectral line width as a function of peak power.
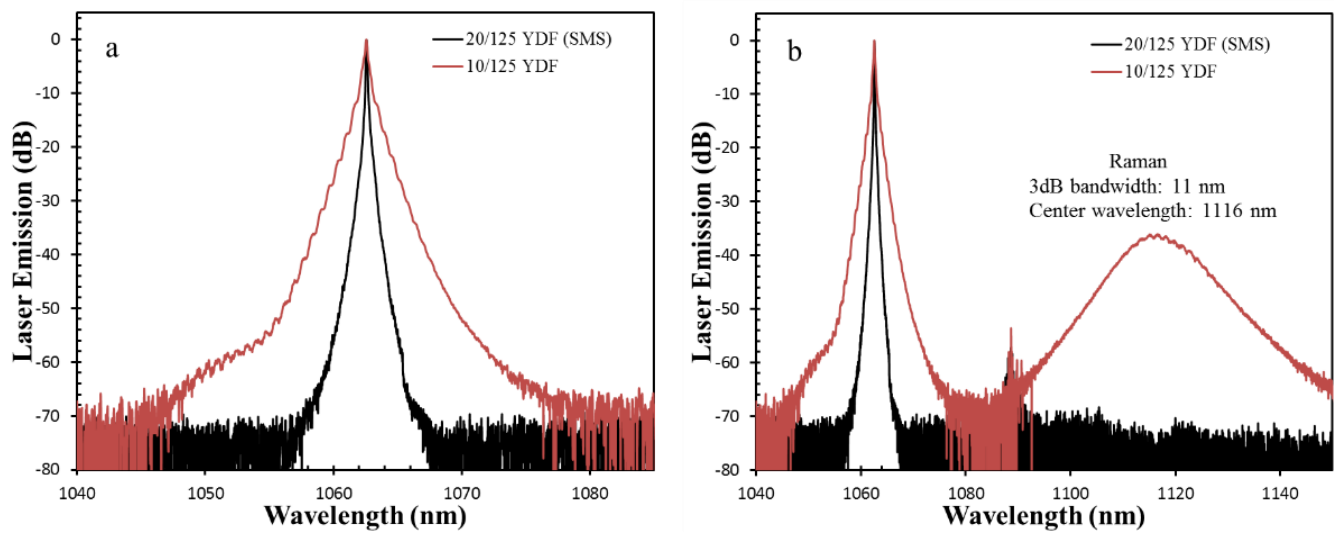

Fig. 7.16 A comparison of the laser emission spectra between the SMS structured laser and the reference laser.

When the emission spectra of two lasers are plotted together in Fig. 7.16(a), we can see a significant difference in the bandwidths between two spectra - the bandwidth at $-10 \mathrm{~dB}$ of the reference laser is $1.12 \mathrm{~nm}$, about 3.5 times broader than that of the SMS-structured laser. The spectrum from the reference laser shows clearly the SPM effect with the ripples coming from interference of the same frequency shifts, resulting from the time derivative of the pulse envelope 
above and below the inflection points. On the contrary, the spectrum of the SMSstructured laser shows hardly any ripple despite its relatively higher peak power. A comparison of the bandwidth between two lasers at three different intensity levels is given in Table 1.

When the emission spectra of two lasers are plotted in an extended spectral range to include Raman spectrum, as shown in Fig. 7.16(b), the difference is more striking. The reference laser spectrum shows a broad Raman emission peak, centered at $1116 \mathrm{~nm}$ with a FWHM of $11 \mathrm{~nm}$. The optical signal to noise ratio of the Raman peak is about $35 \mathrm{~dB}$. On the contrary, the spectrum from the SMSstructured laser shows no Raman emission at all. It is evident, from this comparison that the use of SMS structure effectively inhibits the SRS effect.

Table 7.1: Parameters comparison between SMS laser and a reference laser.

\begin{tabular}{|c|c|c|c|}
\hline & SMS Q-switched laser & Reference laser \\
\hline \multirow{2}{*}{\multicolumn{2}{|c|}{ Peak Power (W) }} & 920 & 800 \\
\hline \multirow{2}{*}{$\begin{array}{c}\text { Bandwidth } \\
(\mathrm{nm})\end{array}$} & $-3 \mathrm{~dB}$ & 0.12 & 0.24 \\
\cline { 2 - 4 } & $-10 \mathrm{~dB}$ & 0.32 & 1.12 \\
\cline { 2 - 4 } & $-20 \mathrm{~dB}$ & 1.16 & 3.52 \\
\hline \multicolumn{2}{r|}{} & 0 & $34 \mathrm{~dB}$ \\
\hline \multicolumn{2}{r|}{ Raman } & \multicolumn{2}{c}{} \\
\hline
\end{tabular}

I expect the pulse width to be shortened with the increase of pump current amplitude. When the pump light intensity is high, the ASE in the gain fiber takes less time to reach the level to bleach the saturable absorber. In addition high pump intensity leads to a larger population inversion in the gain fiber which will deplete faster after the onset of Q-switching. Indeed, when the pulse width is plotted versus the pump power in Fig. 7.17, an asymptotic decrease of the pulse width can be seen. Since the average output power also increases with the increase of 
pump power, the peak power is expected to increase at a faster rate, which is also confirmed in Fig. 7.17.

Since the output fiber is still a single-mode fiber with core/cladding diameters of $10 / 130 \mu \mathrm{m}$ and a NA of 0.075 , the laser output beam retains a Gaussian profile, similar to the results of a CW fiber laser with an SMS structure presented in the previous section.

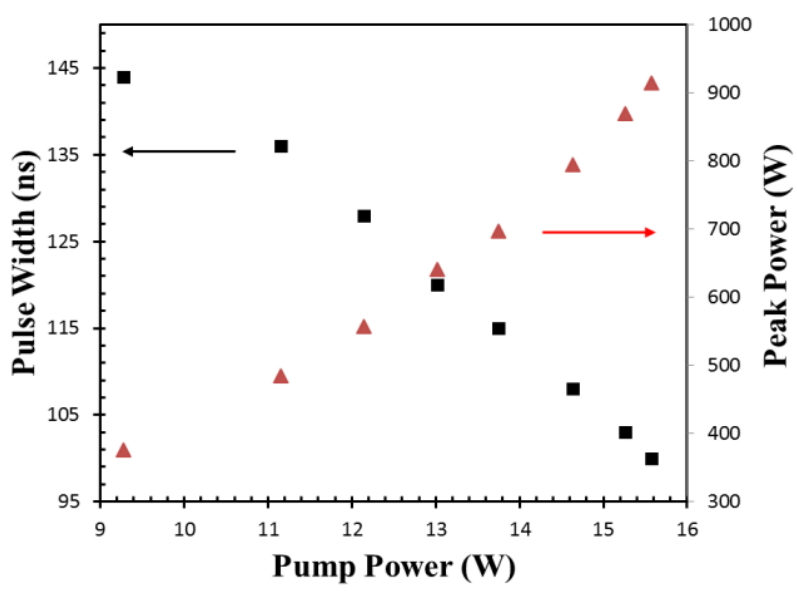

Fig. 7.17 The SMS-structured laser pulse width (black); and peak power (red) as a function of average pump power.

\subsection{Summary}

In this chapter, I have presented a new type of SMS filter at $1046 \mathrm{~nm}$, one high efficiency SMS CW fiber lasers and one SMS Q-switched fiber laser.

Firstly, I have successfully demonstrated an SMS filter which has a mode dispersion difference maximum wavelength at $1046 \mathrm{~nm}$, right at the $\mathrm{Yb}$-doped fiber laser emission wavelength region. The filter used a commercially-available LMA fiber with a step-indexed core. The transmission spectrum was calculated based on the assumption of two mode interference, which matches well with the measured spectrum. The strain and temperature dependence of the transmission spectrum were also characterized which show many potential applications of this filter. 
Secondly, an SMS Yb-doped fiber laser operating at $1088 \mathrm{~nm}$ is also been demonstrated. An LMA gain fiber of $1.5 \mathrm{~m}$ in length was spliced between two FBGs, inscribed in two SMFs respectively, to form an SMS filter and a laser cavity. Using a $976 \mathrm{~nm}$ laser diode as pump source, the laser generated an output power up of $38.5 \mathrm{~W}$ with a slope efficiency of $70 \%$. Output beam quality was characterized and proved to be in a single-transverse-mode. This work proved that one can take the advantage of high cladding absorption of a large core in the gain fiber while avoiding the beam quality deterioration with the SMS structure.

Thirdly, I further designed an all-fiber Q-switched Yb-doped laser with a peak power close to $1 \mathrm{~kW}$ using an SMS structure. The laser achieved a reasonably high slope efficiency of 59\%. More importantly, it proves that the SMS structure is an effective design to inhibit non-linear effects - the SPM-induced linewidth is significantly reduced and stimulated Raman emission is completely eliminated. Among many methods developed for achieving high peak power and high pulse energy, our results showed a new design for scaling-up the peak power to multiple $\mathrm{kW}$ level while maintaining good beam quality and keeping the non-linear effects at low level. 


\section{Chapter 8}

\section{Summary and perspectives}

\subsection{The main feature of my DS mode-locked lasers}

The main features of DS mode-locked fiber lasers described in this dissertation are:

- The DS fiber laser used all-fiber, all-PM structure and NALM as equivalent SA to achieve self-starting and environmentally-stable DS mode-locking. It overcomes the drawback of NPE mode-locking.

- The laser generated significantly high pulse energy of $56 \mathrm{~nJ}$, which is 3.5 times higher than previously reported pulse-energy [35]. It overcomes low energy limitation of SESAM mode-locked fiber lasers.

- The laser generated stable, high energy DS while limiting Raman emission to less than a few percent in the output.

\subsection{The performance of my DS mode-locked lasers}

In this dissertation, two configurations of all-fiber all-PM passively DS modelocked fiber lasers have been presented. One is based on semiconductor saturable absorber mirror (SESAM); the other is using nonlinear amplified loop mirror (NALM).

In chapter four, an all-fiber, environmentally-stable and ultra-compact ANDi fiber laser by using one MFA to launch light onto the SESAM was demonstrated. A PM fiber loop mirror is used as a multi-functional component to realize the design of compact size. The purpose of using an MFA instead of a lens to launch light onto the SESAM is not only to achieve all-fiber structure, but also to enlarge the beam spot on the SESAM for the sake of high pulse energy. Self-starting and 
stable dissipative soliton mode-locking operation is achieved with $1.7 \mathrm{~nJ}$ pulse energy and 22 ps pulse width. The power stability test for over 4 hours confirms the environmentally-stable property of this laser.

Despite the good stability of this SESAM mode-locked fiber laser, its output energy is still limited to less than $2 \mathrm{~nJ}$. Therefore, other alternative SAs have to be employed for the purpose of achieving both environmentally-stable output and higher single pulse energy. In chapter five and six, I presented that stable DS mode-locking can be achieved in a long cavity ring laser with a NALM. Different output energy has been achieved by different cavity length. $32 \mathrm{~nJ}, 615$ fs dechirped pulse width were obtained with the Raman signal suppressed below -20 $\mathrm{dB}$ in the $81 \mathrm{~m}$ long cavity. This mode-locking is self-starting and the modelocked pulse train shows excellent stability, which is confirmed by the RF spectrum and by a power stability test over 24 hours. Further increasing the cavity length to $143 \mathrm{~m}$ can lead to stable $56.8 \mathrm{~nJ}$ single dissipative soliton with $1.4 \mathrm{MHz}$ repetition rate, which can also be dechirped back to 750 fs by a pair of volume gratings.

\subsection{The new phenomena observed in the NALM mode-locked lasers}

While trying to further scale-up the pulse energy of NALM mode-locked fiber lasers, I also found out some interesting new phenomena which are related to the working principle of the NALM loop.

Firstly, an NALM-based mode-locked fiber laser with an overall cavity length of $81 \mathrm{~m}$ was constructed. By increasing the pump power, a working regime transition from noise-like to DS was observed. This transition is accompanied by a $16 \mathrm{~dB}$ drop of the Raman peak. The transition from noise-like to DS can be explained by the transfer function of NALM. At low input peak power, the NALM modulation is not sufficient to cut out the two pulse wings so the laser produces noise-like pulses. At high input peak power, the higher modulation provided sufficient saturable absorption function for the laser to switch to DS 
operation. The reduction of the Raman signal accompanied with this transition can be explained by the difference of the peak power in two regimes.

Secondly, the cavity length was increased by adding $12 \mathrm{~m}$ PM 980 fiber and $50 \mathrm{~m}$ PLMA 10/125-M fiber. A unique multi-pulsing phenomenon in the NALM-based mode-locked fiber laser was observed. Stable $56.8 \mathrm{~nJ}$ single dissipative soliton was generated at an $1.4 \mathrm{MHz}$ repetition rate. Further increasing the gain inside NALM will lead to a pulse breaking to form two stable solitons whose separation is determined by the round-trip time of the NALM loop. The temporal spacing between the split pulses can be step-tuned by changing the gain of NALM. Such multi-pulsing not only possesses high energy but also has unique dynamics which does not fall into any types of soliton reported, such as soliton molecules, soliton rains or harmonic mode-locking. The RF spectra of different separation states were measured which proved the excellent stability of such multi-pulsing states. Such stability is resulting from all-fiber and all polarization maintaining configuration. The laser may have many penitential applications since it has high pulse energy and it can operate in both single soliton and multi-pulsing.

\subsection{A summary of the simulation results}

The forming of a stable dissipative soliton (DS) is the result of a balance between gain, loss, nonlinearity and dispersion. All of these factors could be modified by the nonlinear Schrodinger equation (NLSE). In chapter three, a numerical model for DS mode-locked fiber laser is presented. Several parameters can affect the performance of DS laser including nonlinear phase shift accumulated by the pulse, spectral filter bandwidth, and group-velocity dispersion. Trends in the laser performance as varying these parameters are presented, which can guide the experimental design of DS fiber lasers.

The simulations showed that smaller filter bandwidth and higher output coupler ratio can lead to more stable mode-locking pulses in the design of ANDi modelocked fiber lasers. When these two parameter are fixed, one can further extend 
the cavity length to get higher energy output, as long as the cavity can still produce stable DS solitons.

\subsection{SMS fiber filters and lasers}

Using large-mode-area (LMA) fibers is an effective way to overcome the nonlinearity which limits the further scale-up of the pulse energy. Therefore, learning the property of single-mode- multimode- single-mode (SMS) structure is essential for achieving higher energy DS mode-locked lasers. In chapter eight, I designed and demonstrated a new type of SMS filter at $1046 \mathrm{~nm}$, one high efficiency SMS CW fiber laser and one SMS Q-switched fiber laser which can effectively inhibited the SRS and significantly reduced SPM.

An SMS fiber filter using a step-indexed LMA fiber, which has a critical wavelength at $1046 \mathrm{~nm}$, was firstly designed and demonstrated. A broad transmission peak or trough at $1046 \mathrm{~nm}$ is very sensitive to strain or temperature variation. The interference fringes on either side of the critical wavelength show blue or red shift, respectively, when the filter is subjected to strain and temperature changes. The transmission spectrum was simulated based on the interference of two modes, $\mathrm{LP}_{01}$ and $\mathrm{LP}_{02}$, and their propagation constant difference. The simulated spectrum matches reasonably well with the experimental spectrum. The filter can be used as a variable output coupler or for fiber optic sensors.

An all-fiber, high efficiency Yb-doped laser operating at $1088 \mathrm{~nm}$ with an SMS structure is presented secondly. An LMA gain fiber of $1.5 \mathrm{~m}$ length, with 20/130 $\mu \mathrm{m}$ core/cladding diameters was used to increase the absorption, and a diffractionlimited Gaussian output beam was obtained from the single-mode output fiber. The laser generated an output power up to $38.5 \mathrm{~W}$ with a slope efficiency of $70 \%$. The output beam qualities, with and without SMS structure, were compared, which showed that the fiber laser with the SMS structure can achieve high gain, short fiber length, and excellent beam quality. 
The third SMS design presented is a Q-switched Yb-doped fiber laser in a SMS structure to inhibit fiber nonlinear effects. The laser-generated Q-switched pulses with a peak power close to $1 \mathrm{~kW}$ (pulse width and energy of $100 \mathrm{~ns}$ and $92 \mu \mathrm{J}$, respectively). The output spectrum of this laser was compared with that of another Q-switched Yb-doped fiber laser built in a conventional configuration with similar output peak power. The results showed that the SMS Q-switched laser completely inhibited the stimulated Raman scattering and significantly reduced self-phase modulation.

\subsection{Future work}

Although 56 nJ DS output has been achieved in this work, further energy scale-up is hindered by the nonlinear effect. In chapter 6, SPM has been proved to be the main cause to the pulse splitting. In chapter 5, SRS has also been proved to the main factor to the energy scale-up in a long cavity mode-locked fiber laser. Therefore, future work should be concentrated on overcoming these limitations. Replacing all single-mode fiber with LMA one could be an effective method. Recently, Chen et.al reported the direct generation of $0.4 \mu \mathrm{J}, 7 \mathrm{~kW}$ ultrabroadband picosecond noise-like pulses from an Yb-doped all-fiber oscillator based on dual NOLMs by designing the laser cavity with all-LMA fiber [105]. However, two polarization controllers were involved in their design and $0.4 \mu \mathrm{J}$ pulse energy were achieved under noise-like pulse condition. If the fiber in the NALM laser described in this work is also replaced by the LMA fiber, there is a good possibility for the output pulse energy to be increase to $\mu \mathrm{J}$ level.

Another way to achieve $\mu \mathrm{J}$ level pulse energy is using optical amplification. The NALM-based mode-locked fiber laser has proved itself to be an excellent seed laser for further amplification. The energy threshold for the material processing of glass, ceramics and semiconductors is around $\mu \mathrm{J}$ level. Thus, the laser's energy needs to be amplified by at least 100 times to reach that requirement. This can be achieved by applying one or two chirped pulse amplifiers (CPA). 
Another possible future work for this laser is supercontinuum generation. If the pulse of $56 \mathrm{~nJ}$ can be dechirped into 600 fs by a chirped FBG, the peak power could easily reach $30 \mathrm{~kW}$ (counting the loss from the CFBG and circulator). Thus, an all-fiber and cost-effective supercontinuum generation light source can be easily developed by combining this mode-locked fiber laser and a piece of PCF. As different fiber laser systems with higher stability are developed, the number of application will also continue to grow up, and in the future, mode-locked fiber lasers will find more applications in much wider commercial markets. 


\section{References}

[1] I. Hartl, G. Imeshev, L. Dong, G. C. Cho \& M. E. Fermann, "Ultra-compact dispersion compensated femtosecond fiber oscillators and amplifiers," Paper CThG1 in Conf. on Lasers Electro-Optics (OSA, 2005).

[2] M. O. Pedersen, et al. "High power polarization maintaining supercontinuum source," Paper p1-1 in Las. Electro-Opt. 2007 Inter. Quant. Electron. Conf. (2007).

[3] I. Hartl, et al. "Cavity-enhanced similariton Yb-fiber laser frequency comb: $3 \times 1014 \mathrm{~W} / \mathrm{cm}^{2}$ peak intensity at $136 \mathrm{MHz}$," Opt. Lett. 32, 2870-2872 (2007).

[4] J. M. Dudley, G. Genty \& S. Coen, "Supercontinuum generation in photonic crystal fiber," Rev. Mod. Phys. 78, 1135-1184 (2006).

[5] M. Nagai, E. Matsubara, M. Ashida, J. Takayanagi \& H. Ohtake, "Generation and detection of $\mathrm{THz}$ pulses with a bandwidth extending beyond $4 \mathrm{THz}$ using a sub-picosecond Yb-doped fiber laser system," IEEE Transactions on Terahertz Science and Technology, 4, 440-446 (2014).

[6] P. Grelu, \& N. Akhmediev, "Dissipative solitons for mode-locked lasers," Nature Photonics, 6, 84-92 (2012).

[7] H. H. Hopkins \& N. S. Kapany, "A flexible fibrescope, using static scanning," Nature 173, 39-41 (1954).

[8] C. K. Kao, "Nobel Lecture: sand from centuries past: send future voices fast," Rev. Mod. Phys. 82, 2299-2303 (2010).

[9] E. Snitzer, "Optical maser action of $\mathrm{Nd}^{+3}$ in a barium crown glass," Phys. Rev. Lett. 7, 444-446 (1961).

[10] E. Desurvire, J. R. Simpson \& P. C. Becker, "High-gain erbium-doped traveling-wave fiber amplifier," Opt. Lett. 12, 888-890 (1987).

[11] R. Mears, L. Reekie, I. M. Jauncey \& D. N. Payne, "Low-noise erbium-

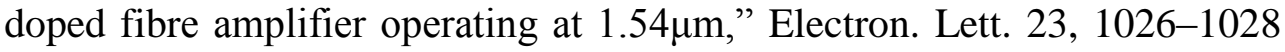
(1987).

[12] M. E. Fermann \& I. Hartl, "Ultrafast fiber lasers," Nature Photonics 7, 868874 (2013).

[13] W.H. Renninger and F.W. Wise, "Dissipative soliton fiber laser," Chapter 4, 1st edition, Wiley-VCH Verlag GmbH \& Co. KGaA 2012.

[14] M. Fermann, M. Hofer, F. Haberl \& S. P. Craig-Ryan, "Femtosecond fiber laser," Electron. Lett. 26, 1737-1738 (1990). 
[15] K. Tamura, E. P. Ippen, H. A. Haus \& L. E. Nelson, "77-fs pulse generation from a stretched-pulse mode-locked all-fiber ring laser," Opt. Lett. 18, 1080-1082 (1993).

[16] A. C. Tien, S. Backus, H. Kapteyn, M. Murnane \& G. Mourou, "Short-pulse laser damage in transparent materials as a function of pulse duration," Phys. Rev. Lett. 82, 3883-3886 (1999).

[17] http://www.cmxr.com/index.php

[18] N. Akhmediev \& A. Ankiewicz. Solitons, nonlinear pulses and beams Ch. 2 (Chapman and Hall, 1997).

[19] S. Kelly. "Characteristic sideband instability of periodically amplified average soliton," Electronics Letters, 28, 806-807 (1997).

[20] A. Chong, W. H. Renninger and F. W. Wise, "All-normal-dispersion femtosecond fiber laser with pulse energy above $20 \mathrm{Nj}$," Opt. Lett. 32, 24082410 (2007).

[21] Q. Bao, H. Zhang, Y. Wang, Z. Ni and D. Y. Tang, "Atomic - layer graphene as a saturable absorber for ultrafast pulsed lasers," Advanced Functional Materials, 19, 3077-3083 (2009).

[22] L. Zhao, D. Tang, H. Zhang, T. Cheng, H. Tam and C. Lu, "Dynamics of gain-guided solitons in an all-normal-dispersion fiber laser," Opt. Lett. 32, 1806-1808 (2007).

[23] A. Chong, J. Buckley, W. H. Renninger and F. W. Wise, "All-normaldispersion femtosecond fiber laser," Opt. Exp. 14, 10095-10100 (2006).

[24] E. Picholle, C. Montes, C. Leycuras, O. Legrand \& J. Botineau, "Observation of dissipative superluminous solitons in a Brillouin fiber ring laser," Phys. Rev. Lett. 66, 1454-1457 (1991).

[25] E. V. Vanin et al. "Dissipative optical solitons," Phys. Rev. A 49, 28062811 (1994).

[26] O. Okhotnikov, T. Jouhti, J. Konttinen, S. Karirinne and M. Pessa, "1.5- $\mu$ m monolithic GaInNAs semiconductor saturable-absorber mode locking of an erbium fiber laser," Opt. Lett. 28, 364-366 (2003).

[27] S. Y. Set, H. Yaguchi, M. Jablonski, Y. Tanaka, Y. Sakakibara, A. G. Rozhin, M. Tokumoto, H. Kataura, Y. Achiba and K. Kikuchi, "A noise suppressing saturable absorber at $1550 \mathrm{~nm}$ based on carbon nanotube technology," Optical Fiber Communication Conference, 132-137 (2003).

[28] L. Zhao, D. Tang, H. Zhang, X. Wu, Q. Bao and K. P. Loh, "Dissipative soliton operation of an ytterbium-doped fiber laser mode locked with atomic multilayer graphene," Opt. Lett. 35, 3622-3624 (2010). 
[29] V. Matsas, T. Newson, D. Richardson and D. Payne, "Selfstarting passively mode-locked fiber ring soliton laser exploiting nonlinear polarisation rotation," Electron. Lett. 28, 1391-1393 (1992).

[30] F. Ilday, F. Wise \& T. Sosnowski, "High-energy femtosecond stretchedpulse fiber laser with a nonlinear optical loop mirror," Opt. Lett. 27, 15311533 (2002).

[31] C. Aguergaray, N. G. R. Broderick, M. Erkintalo, J. S. Y. Chen, and V. Kruglov, "Mode-locked femtosecond all-normal all-PM Yb-doped fiber laser using a nonlinear amplifying loop mirror," Opt. Express 20(10), 10545-10551 (2012).

[32] K. Özgören and F. Ilday, "All-fiber all-normal dispersion laser with a fiberbased Lyot filter,” Opt. Lett. 35, 1296-1298 (2010).

[33] K. Kieu and F. Wise, "All-fiber normal-dispersion femtosecond laser," Opt. Express, 16, 11453 (2008).

[34] R. Gumenyuk, I. Vartiainen, H. Tuovinen and O. G. Okhotnikov, "Dissipative dispersion-managed soliton $2 \mu \mathrm{m}$ thulium/holmium fiber laser," Opt. Lett. 36, 609-611 (2011).

[35] M. Erkintalo, C. Aguergaray, A. Runge, and N. G. R. Broderick, "Environmentally stable all-PM all-fiber giant chirp oscillator," Opt. Express 20(20), 22669-22674 (2012).

[36] C. Aguergaray, R. Hawker, A. Runge, M. Erkintalo and N. G. R. Broderick, "120 fs, $4.2 \mathrm{~nJ}$ pulses from an all-normal-dispersion, polarizationmaintaining, fiber laser,” Appl. Phy. Lett. 103(12), 121111 (2013).

[37] D. S. Kharenko, E. V. Podivilov, A. A. Apolonski, and S. A. Babin, "20 nJ 200 fs all-fiber highly-chirped dissipative soliton oscillator," Opt. Lett. 37, 4104-4106 (2012).

[38] A. E. Bednyakova, S. A. Babin, D. S. Kharenko, E. V. Podivilov, M. P. Fedoruk, V. L. Kalashnikov, and A. Apolonski, "Evolution of dissipative solitons in a fiber laser oscillator in the presence of strong Raman scattering," Opt. Express 21, 20556-20564 (2013).

[39] A. Kumar, R. K. Varshney, and P. Sharma. "Transmission characteristics of SMS fiber optic sensor structures." Optics communications 219.1: 215-219 (2003).

[40] W. S. Mohammed, P. W. E. Smith and Xijia Gu, "All-fiber multimode interference bandpass filter," Opt. Lett., vol. 31, no. 17, pp. 2547-2549, Sept. 2006.

[41] S. M. Tripathi, A. Kumar, R. K. Varshney, Y. B. P. Kumar, E. Marin, and J.-P. Meunier, "Strain and temperature sensing characteristics of singlemode-multimode-single-mode structures," J. Lightw. Technol., 27, 2348-2356 (2009). 
[42] Y. Zhang, X. Tian, L. Xue, Q. Zhang, L. Yang, and B. Zhu, "Super-high sensitivity of fiber temperature sensor based on leaky-mode bent SMS structure,” IEEE Photon. Technol. Lett., 25, 560-563 (2013).

[43] H. Xue, H. Meng, W. Wang, R. Xiong, Q. Yao, and B. Huang, "Singlemode-multimode fiber structure based sensor for simultaneous measurement of refractive index and temperature," IEEE Sensors Journal., 13, 4220-4223 (2013).

[44] S. M. Tripathi, A. Kumar, E. Marin, and J.-P. Meunier, "Critical wavelength in the transmission spectrum of SMS fiber structure employing $\mathrm{GeO}^{2-}$ doped multimode fiber" IEEE Photon. Technol. Lett. 22, 799-801 (2010).

[45] E. Salik, M. Medrano, G. Cohoon, J. Miller, C. Boyter, and J. Koh, "SMS Fiber Sensor Utilizing a Few-Mode Fiber Exhibits Critical Wavelength Behavior,” IEEE Photon. Technol. Lett. 24, 593-595 (2012).

[46] D. J. Richardson, J. Nilsson, and W. A. Clarkson, "High Power fiber lasers: current status and future," J. Opt. Soc. Am. B 27, 63-92 (2010).

[47] J. He, S. Du, Z. Wang, Z. Wang, J. Zhou and Q. Lou, "Linearly-polarized short-pulse AOM Q-switched $978 \mathrm{~nm}$ photonic crystal fiber laser," Opt. Express 21, 29240-29245 (2013).

[48] H. L. Offerhaus, N. G. Broderick, D. J. Richardson, R. Sammut, J. Caplen and L. Dong, "High-energy single-transverse-mode Q-switched fiber laser based on a multimode large-mode-area erbium-doped fiber," Opt. Lett. 23, 1683-1685 (1998).

[49] U. Griebner, R. Koch, and H. Schonnagel, "Efficient laser operation with nearly diffraction-limited output from a diode-pumped heavily Nd-doped multimode fiber," Opt. Lett. 21, 266-268 (1996).

[50] A. Polynkin, P. Polynkin, A. Schülzgen, M. Mansuripur, and N. Peyghambarian, "Watts-level, short all-fiber laser at $1.5 \mathrm{~mm}$ with a large core and diffraction-limited output via intracavity spatial-mode filtering," Opt. Lett. 30, 403-405 (2005).

[51] P. Wang, L. J. Cooper, J. K. Sahu, and W. A. Clarkson, "Efficient singlemode operation of a cladding pumped ytterbium-doped helical-core fiber laser," Opt. Lett. 31, 226-228 (2006).

[52] L. Dong, J. Li, and X. Peng, "Bend-resistant fundamental mode operation in ytterbium-doped leakage channel fibers with effective areas up to 3160 $\mu \mathrm{m}^{2}$," Opt. Express 14, 11512-11519 (2006).

[53] X. Zhu, A. Schulzgen, H. Li, L. Li, Q. Wang, S. Suzuki, V. L. Temyanko, J. V. Moloney, and N. Peyghambarian, "Single-transverse-mode output from a fiber laser based on multimode interference," Opt. Lett., 33, 908-910, (2008). 
[54] X. Zhu, A. Schulzgen, H. Li, L. Li, V.L. Temyanko, J.V. Moloney, N. Peyghambarian, "High-Power Fiber Lasers and Amplifiers Based on Multimode Interference," IEEE Journal of Selected Topics in Quantum Electronics, 15, 71-78, (2009).

[55] Thual, O. \& Fauve S. Localized structures generated by subcritical instabilities. J. Phys. France 49, 1829-1833 (1988).

[56] Soto-Crespo, J. M., Akhmediev, N. \& Afanasjev, V. Stability of the pulselike solutions of the quintic complex Ginzburg-Landau equation. J. Opt. Soc. Am. 13, 1439-1449 (1996).

[57] Kutz, J. N. Mode-locked soliton lasers. SIAM Rev. 48, 629-678 (2006).

[58] J. Limpert, T. Schreiber, T. Clausnitzer, K. Zöllner, H.-J. Fuchs, E.-B. Kley, H. Zellmer, A. Tünnermann. High-power femtosecond Yb-doped fiber amplifier. Opt. Express, 10(14), 628-638 (2002).

[59] F. O. Ilday, J. R. Buckley and F. W. Wise. Self-Similar Evolution of Parabolic Pulses in a Laser. Phy. Rev. Lett., 92(213902) (2004).

[60] P. V. Mamyshev and S. V. Chernikov. Ultrashort-pulse propagation in optical fibers. Opt. Lett. 15(19), 1076-1078 (1990).

[61] http://www.batop.de

[62] N.J. Doran and David Wood, "Nonlinear-optical loop mirror," Opt. Lett., 13(1), 56-58 (1988).

[63] M. E. Fermann, F. Haberl, M. Hofer and H. Hochreiter. "Nonlinear amplifying loop mirror," Opt. Lett., 15(13), $752-754$ (1990).

[64] O. G. Okhotnikov, L. Gomes, N. Xiang, T. Jouhti, and A. B. Grudinin. "Mode-locked ytterbium fiber laser tunable in the $980-1070 \mathrm{~nm}$ spectral range." Opt. Lett., 28(17): 1522-1524 (2003).

[65] L. Gomes, L. Orsila, T. Jouhti, and O. G. Okhotnikov. "Picosecond SESAM-based ytterbium mode-locked fiber lasers." IEEE Journal of Selected Topics in Quantum Electronics, 10(1): 129-136 (2004).

[66] X. Tian, M. Tang, P. Shum, Y. Gong, C. Lin, S. Fu, and T. Zhang. "Highenergy laser pulse with a submegahertz repetition rate from a passively mode-locked fiber laser." Opt. Lett., 34(9): 1432-1434 (2009).

[67] P. C. Becker, N. A. Olsson and J. R. Simpson. "Erbium-Doped Fiber Amplifiers: Fundamentals and Technology", 1st ed., Academic Press, San Diego (1999).

[68] E. Desurvire, D. Bayart, B. Desthieux and S. Bigo. "Erbium-Doped Fiber Amplifiers", 1st ed., John Wiley \& Sons Inc., New York (2002).

[69] V. Cautaerts, D. J. Richardson, R. Paschotta, and D. C. Hanna. "Stretched pulse $\mathrm{Yb}^{3+}$ : silica fiber laser," Opt. Lett., 22, 316-318 (1997). 
[70] L. Lefort, J. H. V. Price, D. J. Richardson and J. Weston. "Practical low noise stretched-pulse $\mathrm{Yb}^{3+}$-doped fiber laser," Opt. Lett., 27, 291-293 (2002).

[71] H. Lim, F. O. Ilday, and F. W. Wise. "Generation of $2 \mathrm{~nJ}$ pulses from a femtosecond ytterbium fiber laser," Opt. Lett., 28, 660-662 (2003).

[72] A. Chong, W. H. Renninger and F. W. Wise. "Properties of normaldispersion femtosecond fiber lasers," J. Opt. Soc. Am. B, 25(2), 1522-1524 (2003).

[73] C. Aguergaray, A. Runge, M. Erkintalo, and N. G. R. Broderick, "Ramandriven destabilization of mode-locked long cavity fiber lasers: fundamental limitations to energy scalability," Opt. Lett. 38, 2644-2646 (2013).

[74] I. Hartl, G. Imeshev, L. Dong, G. C. Cho, and M. E. Fermann, "UltraCompact Dispersion Compensated Femtosecond Fiber Oscillators and Amplifiers," in Technical Digest (CD) (Optical Society of America, 2005), CThG1.

[75] C. Nielsen, B. Ortaç, T. Schreiber, J. Limpert, R. Hohmuth, W. Richter, and A. Tünnermann, "Self-starting self-similar all-polarization maintaining Ybdoped fiber laser," Opt. Express 13, 9346-9351 (2005).

[76] S. Masuda, S. Niki, and M. Nakazawa, "Environmentally stable, simple passively mode-locked fiber ring laser using a four-port circulator," Opt. Express 17, 6613-6622 (2009).

[77] L. Zhang, J. Zhou, Z. Wang, X. Gu and Y. Feng, " SESAM Mode-Locked, Environmentally Stable, and Compact Dissipative Soliton Fiber Laser," IEEE Photonics Tech. Lett. 26, 1314-1316 (2014).

[78] L. M. Zhao, A. C. Bartnik, Q. Q. Tai, and F. W. Wise, "Generation of $8 \mathrm{~nJ}$ pulses from a dissipative-soliton fiber laser with a nonlinear optical loop mirror," Opt. Lett., Vol. 38, no. 11, pp. 1942-1944 (2013).

[79] P. Grelu, N. Akhmediev, "Dissipative solitons for mode-locked lasers," Nature Photonics 6, 84-92 (2012).

[80] P. Grelu and J. M. Soto-Crespo, Temporal soliton 'molecules' in modelocked lasers: Collisions, pulsations and vibrations. Lect. Notes Phys. 751, 137-173 (2008).

[81] J. P. Gordon, "Interaction forces among solitons in optical fibers," Opt. Lett. 8(11), 596-598 (1983).

[82] B. A. Malomed, "Bound solitons in the nonlinear Schrödinger-GinzburgLandau equation," Phys. Rev. A 44, 6954-6957 (1991).

[83] J. M. Soto-Crespo, M. Grapinet, P. Grelu and N. Akhmediev, "Bifurcations and multiple-period soliton pulsations in a passively mode-locked fiber laser," Phys. Rev. E 70, 066612 (2004). 
[84] P. Grelu, N. Akhmediev, "Group interactions of dissipative solitons in a laser cavity: the case of 2+1," Opt. Express 12(14), 3184-3189 (2004).

[85] M. Grapinet and P. Grelu, "Vibrating soliton pairs in a mode-locked laser cavity," Opt. Lett. 31(14), 2115-2117 (2006).

[86] S. Chouli and P. Grelu, "Rains of solitons in a fiber laser," Opt. Express 17(14), 11776-11781 (2009).

[87] S. Chouli and P. Grelu, "Soliton rains in a fiber laser: An experimental study,” Phys. Rev. A 81, 063829 (2010).

[88] J. Nathan Kutz, B. C. Collings, K. Bergman, and W. H. Knox, "Stabilized pulse spacing in soliton lasers due to gain depletion and recovery," IEEE J. Quantum Elect. 34(9), 1749-1756 (1998).

[89] L. M. Zhao, D. Y. Tang, T. H. Cheng, et al., "Generation of multiple gainguided solitons in a fiber laser," Opt. Lett. 32(11), 1581-1583 (2007).

[90] J. A. Buck, Fundamental of Optical Fibers, 2nd ed. Hoboken, NJ, USA: Wiley, 2004, ch. 3.

[91] A. Kersey et al., "Fiber grating sensors," J. Lightw. Technol., 15(8), 14421463 (1997).

[92] D. J. Richardson, J. Nilsson, and W. A. Clarkson, "High power fiber lasers: current status and future perspective," J. Opt. Soc. Am. B 27, B63-B92 (2010).

[93] W. Shi, Q. Fang, X. Zhu, R. A. Norwood, and N. Peyghambarian, "Fiber lasers and their applications," Appl. Opt. 53, 6554-6568 (2014).

[94] M. Hu, B. He, H. Liu, Y. Yang, Y. Zheng, X. Chen, L. Zhang, and J. Zhou, "High-peak power, all-fiber passively Q-switched laser using a Sm-doped fiber saturable absorber," IEEE J. Lightwave Technol. 32, 2510-2515 (2014).

[95] B. N. Upadhyaya, A. Kumar, U. Chakravarty, S. M. Oak, M. R. Shenoy, and K. Thyagarajan, "Analysis of output pulse characteristics in Q-switched Yb-doped fiber laser,” IEEE J. Quantum Electron. 47, 786-794 (2011).

[96] J. Zhao, P. Yan, J. Shu, and S. Ruan, "1 MW peak power, sub-nanosecond master oscillator fiber power amplifier," in Symposium on Photonics Optoelectronics (SOPO) (IEEE, 2011).

[97] A. Malinowski, P. Gorman, C. A. Codemard, F. Ghiringhelli, A. J. Boyland, A. Marshall, M. N. Zervas, and M. K. Durkin, "High-peakpower, high energy, high average power pulsed fiber laser system with versatile pulse duration and shape," Opt. Lett. 38, 4686-4689 (2013).

[98] R. Lehneis, A. Steinmetz, J. Limpert, and A. Tünnermann, “All-fiber pulse shortening of passively Q-switched microchip laser pulses down to sub-200 fs," Opt. Lett. 39, 5806-5809 (2014). 
[99] C. Larsen, M. Giesberts, S. Nyga, O. Fritzau, B. Jungbluth, H. D. Hoffmann, and O. Bang, "Gain-switched all-fiber laser with narrow bandwidth," Opt. Express 21, 12302-12308 (2013).

[100] C. C. Ranaud, H. L. Offerhaus, J. A. Alvarez-Chavez, J. Nilsson, W. A. Clarkson, P. W. Turner, D. J. Richardson, and A. B. Grudinin, "Characteristics of Q-switched cladding-pumped ytterbium-doped fiber lasers with different high-energy fiber designs," IEEE J. Quantum Electron. 37, 199-206 (2001).

[101] J. He, S. Du, Z. Wang, Z. Wang, J. Zhou, and Q. Lou, "Linearly polarized short-pulse AOM Q-switched $978 \mathrm{~nm}$ photonic crystal fiber laser," Opt. Express 21, 29240-29245 (2013).

[102] D. B. S. Soh, S. E. Bisson, B. D. Patterson, and S. W. Moore, "High power all-fiber passively Q-switched laser using a doped fiber as a saturable absorber: numerical simulations," Opt. Lett. 36, 2536-2538 (2011).

[103] Y. Lu and X. Gu, "Kilowatt peak power pulses from a passively Q-witched $\mathrm{Yb}$-doped fiber laser with a smaller-core $\mathrm{Yb}$-doped fiber as a saturable absorber," IEEE Photon. J. 6, 1501207 (2014).

[104] Y. Lu and X. Gu, "All-fiber passively Q-switched fiber laser with a Smdoped fiber saturable absorber," Opt. Express 21, 1997-2002 (2013).

[105] H. Chen, S. Chen, Z. Jiang and J. Hou, " $0.4 \mu \mathrm{J}, 7 \mathrm{~kW}$ ultra-broadband noise-like pulse direct generation from an all-fiber dumbbell-shaped laser," Opt. Lett. 40, 5490-5493 (2015). 